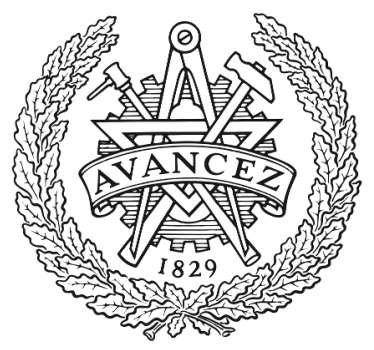

CHALMERS

UNIVERSITY OF TECHNOLOGY

\title{
Towards closed strings as single-valued open strings at genus one
}

Downloaded from: https://research.chalmers.se, 2023-04-26 10:06 UTC

Citation for the original published paper (version of record):

Gerken, J., Kleinschmidt, A., Mafra, C. et al (2022). Towards closed strings as single-valued open strings at genus one. Journal of Physics A: Mathematical and Theoretical, 55(2). http://dx.doi.org/10.1088/1751-8121/abe58b

N.B. When citing this work, cite the original published paper. 
PAPER • OPEN ACCESS

\section{Towards closed strings as single-valued open strings at genus one}

To cite this article: Jan E Gerken et al 2022 J. Phys. A: Math. Theor. 55025401

View the article online for updates and enhancements.
You may also like

Metrology of Zener-based secondary voltage standards

R Chayramy, S Yang, O Power et al.

MORPHOLOGY AND MOLECULAR GAS FRACTIONS OF LOCAL LUMINOUS INFRARED GALAXIES AS A FUNCTION OF INFRARED LUMINOSITY AND MERGER STAGE

K. L. Larson, D. B. Sanders, J. E. Barnes et al.

Basis decompositions and a Mathematica package for modular araph forms Jan E Gerken

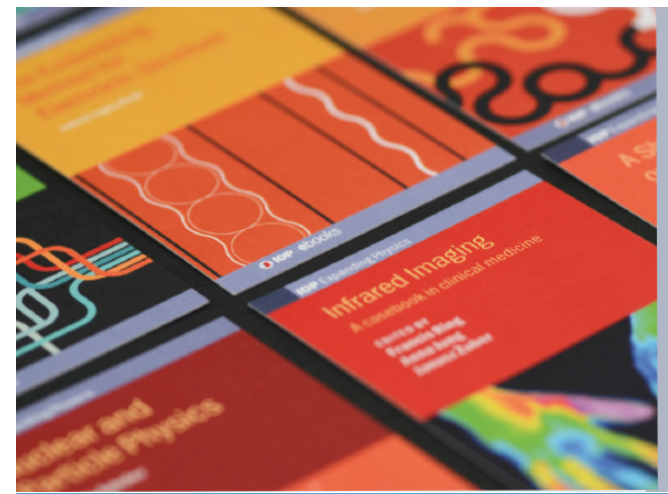

IOP ebooks

Bringing together innovative digital publishing with leading authors from the global scientific community. Start exploring the collection-download the first chapter of every title for free. 


\title{
Towards closed strings as single-valued open strings at genus one
}

\author{
Jan E Gerken ${ }^{1,2}{ }^{\oplus}$, Axel Kleinschmidt ${ }^{2,3}{ }^{(0)}$, \\ Carlos R Mafra ${ }^{4}\left(\mathbb{0}\right.$, Oliver Schlotterer ${ }^{5, *}{ }^{\oplus}$ and \\ Bram Verbeek ${ }^{5}$
}

${ }^{1}$ Department of Mathematical Sciences, Chalmers University of Technology, 41296 Gothenburg, Sweden

2 Max-Planck-Institut für Gravitationsphysik (Albert-Einstein-Institut), Am Mühlenberg 1, DE-14476 Potsdam, Germany

${ }^{3}$ International Solvay Institutes, ULB-Campus Plaine CP231, BE-1050 Brussels, Belgium

${ }^{4}$ STAG Research Centre and Mathematical Sciences, University of Southampton, Highfield, Southampton SO17 1BJ, United Kingdom

5 Department of Physics and Astronomy, Uppsala University, 75108 Uppsala, Sweden

E-mail: gerken@chalmers.se, axel.kleinschmidt@aei.mpg.de, C.R.Mafra@ soton.ac.uk, oliver.schlotterer@physics.uu.se and bram.verbeek@physics.uu.se

Received 10 November 2020, revised 21 January 2021 Accepted for publication 11 February 2021

Published 21 December 2021

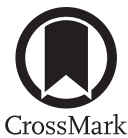

\section{Abstract}

We relate the low-energy expansions of world-sheet integrals in genus-one amplitudes of open- and closed-string states. The respective expansion coefficients are elliptic multiple zeta values (eMZVs) in the open-string case and non-holomorphic modular forms dubbed 'modular graph forms (MGFs)' for closed strings. By inspecting the differential equations and degeneration limits of suitable generating series of genus-one integrals, we identify formal substitution rules mapping the eMZVs of open strings to the MGFs of closed strings. Based on the properties of these rules, we refer to them as an elliptic singlevalued map which generalizes the genus-zero notion of a single-valued map acting on MZVs seen in tree-level relations between the open and closed string.

Keywords: string amplitudes, modular forms, elliptic integrals

S] Supplementary material for this article is available online

*Author to whom any correspondence should be addressed.

Original content from this work may be used under the terms of the Creative Commons Attribution 4.0 licence. Any further distribution of this work must maintain attribution to the author(s) and the title of the work, journal citation and DOI. 
(Some figures may appear in colour only in the online journal)

\section{Introduction}

One-loop amplitudes in string theories are computed from integrals over moduli spaces of punctured genus-one world-sheets. For open and closed strings, the punctures are integrated over a cylinder boundary and the entire torus, respectively, which is often done in a low-energy expansion, i.e. order by order in the inverse string tension $\alpha^{\prime}$. The coefficients of such $\alpha^{\prime}$ expansions involve special numbers and functions which have triggered fruitful interactions between number theorists, particle phenomenologists and string theorists. For instance, elliptic polylogarithms [1,2] and elliptic multiple zeta values (eMZVs) [3] were identified to form the number-theoretic backbone of genus-one open-string integrals [4-6].

For the closed string, the analogous genus-one integrals involve non-holomorphic modular forms [7-9] dubbed modular graph forms (MGFs) [10, 11] which inspired mathematical research lines [12-16]. As a unifying building block shared by open and closed strings, both eMZVs [3, 17] and MGFs [10, 11, 18] can be reduced to iterated integrals over holomorphic Eisenstein series, or iterated Eisenstein integrals. Similar iterated integrals over holomorphic modular forms play a key role in recent progress on the evaluation of Feynman integrals [19-39]. As a main result of this work, we identify infinite families of closed-string integrals, where the appearance of iterated Eisenstein integrals is in precise correspondence with those in open-string $\alpha^{\prime}$-expansions.

More specifically, we give an explicit proposal for a single-valued map at genus one, mapping individual eMZVs to combinations of iterated Eisenstein integrals and their complex conjugates which should be contained in Brown's single-valued iterated Eisenstein integrals $[13,14]$. This generalizes the genus-zero result that the sphere integrals in closed-string tree amplitudes are single-valued versions of the disk integrals in open-string tree amplitudes [40-45]. The notion of single-valued periods [46, 47] and single-valued integration [48, 49] is very general, and in the case of MZVs amounts to evaluating single-valued polylogarithms [50] at unit argument. While the single-valued map for the MZVs in tree-level $\alpha^{\prime}$-expansions has been pinpointed in $[46,48]$, the genus-one studies of single-valued maps from mathematical [12-14] and physical $[51,52]$ viewpoints ${ }^{6}$ have not yet led to a consensus for the single-valued version of individual eMZVs.

Our proposal for single-valued eMZVs can be seen as a correspondence between integration cycles and antimeromorphic forms that is akin to Betti-deRham duality $[49,57,58]$. In a treelevel context, Betti-deRham duality relates the ordering of open-string punctures on a disk boundary to Parke-Taylor factors [40-45] — cyclic products of propagators $\left(\bar{z}_{i}-\bar{z}_{j}\right)^{-1}$ on the sphere. As a genus-one generalization, we spell out certain antielliptic (i.e. antimeromorphic and doubly-periodic) functions on the torus which will be referred to as the Betti-deRham duals $^{7}$ of integration cycles on a cylinder boundary.

It will be important to collect the various eMZVs and MGFs in generating series similar to those in $[18,59-61]$ as the genus-one single-valued map SV is most conveniently described at the level of these generating series. The $\alpha^{\prime}$-expansion of genus-one closed-string integrals — using the techniques of [18] — yields an explicit form of the proposed single-valued map of the eMZVs in open-string integrals. The open-string punctures on a cylinder boundary

\footnotetext{
${ }^{6}$ See $[16,18,53-56]$ for recent progress in identifying single-valued MZVs in the degeneration of dihedral MGFs from closed-string genus-one integrals at the cusp.

${ }^{7}$ We shall use this terminology at genus one even though we are not aware of any explicitly worked out notion of Betti-deRham duality beyond genus zero.
} 
are ordered according to the cycle which is Betti-deRham dual to the additional antielliptic functions in the closed-string integrand. For the purpose of this work, it will be sufficient to place all the open-string punctures on the same cylinder boundary which corresponds to planar genus-one amplitudes: as will be discussed in future work, single-valued non-planar open-string integrals yield the same collection of MGFs as the planar ones. Apart from a characterization via iterated Eisenstein integrals, we will arrive at a closed formula for the singlevalued versions of any convergent eMZV that straightforwardly yields the familiar lattice-sum representations of MGFs.

The main evidence for our proposal for an elliptic single-valued map stems from its consistency with holomorphic derivatives in the modular parameters $\tau$ of the surfaces and the degeneration $\tau \rightarrow i \infty$ of the torus to a nodal sphere. Compatibility with the holomorphic derivative is a simple consequence of recent results on the differential equations of genus-one open-string integrals $[59,60]$ and closed-string integrals [61] in $\tau$. Our antielliptic integrands on the torus ensure that the closed-string differential equations match those of the open string apart from the disappearance of $\zeta_{2}$ as expected from the single-valued map of MZVs. Moreover, the antielliptic integrands are engineered such as to reproduce Parke-Taylor factors in the degeneration $\tau \rightarrow i \infty$. Hence, compatibility of the single-valued maps at genus zero and one is supported by the identification of sphere integrals as single-valued disk integrals [40-45]. The logic of our construction is illustrated in figure 1.

\subsection{Summary of main results}

The main result of this work is the proposal

$$
J_{\vec{\eta}}^{\tau}=\mathrm{SVB}_{\vec{\eta}}^{\tau}
$$

for a single-valued map SV at genus one which relates generating series $B_{\vec{\eta}}^{\tau}$ and $J_{\vec{\eta}}^{\tau}$ of openand closed-string integrals, respectively, see (3.35). As summarized in figure 1, this induces an SV action on the eMZVs in the $\alpha^{\prime}$-expansion of the cylinder integrals $B_{\vec{\eta}}^{\tau}$ to be defined in (3.1). By comparing coefficients of dimensionless Mandelstam invariants $\alpha^{\prime} k_{i} \cdot k_{j}$ and formal expansion variables $\eta_{j}$, SV maps each eMZV generated by $B_{\vec{\eta}}^{\tau}$ to combinations of MGFs at the same order in the analogous expansion of the torus integrals $J_{\vec{\eta}}^{\tau}$ in (3.13). The integrands of $B_{\vec{\eta}}^{\tau}$ and $J_{\vec{\eta}}^{\tau}$ are assembled from combinations of doubly-periodic Kronecker-Eisenstein series $\varphi_{\vec{\eta}}^{\tau}$ in (2.13) known from [18, 59-61] and antielliptic functions $\overline{V(\ldots \mid \tau)}$ that we introduce in (3.8) as tentative Betti-deRham duals of integration cycles on a cylinder boundary.

A key motivation and evidence for this construction stems from the degeneration limit $\tau \rightarrow i \infty$ of the series $B_{\vec{\eta}}^{\tau}$ and $J_{\vec{\eta}}^{\tau}$. Within this limit, genus-zero integrals similar to those in open- and closed-string tree-level amplitudes are recovered, the latter being related by the single-valued map of MZVs [40-45]. The leading terms of the eMZVs in the $\tau \rightarrow i \infty$ limit of $B_{\vec{\eta}}^{\tau}$ are certain Laurent polynomials in the modular parameter $\tau$ of the cylinder with MZVs in its coefficients. As visualized in the lower part of figure 1, the known single-valued map sv of MZVs [46, 48] is conjectured to yield the analogous Laurent polynomials in the degeneration limit $\tau \rightarrow i \infty$ of the torus integrals $J_{\vec{\eta}}^{\tau}$. This is made precise in the conjecture (3.23)—a central prerequisite for (1.1) — which generalizes earlier observations in [51, 52] and has been proven at the leading orders in the formal expansion variable $\eta_{j}$ at two points [16].

The earlier proposal for an elliptic single-valued map 'esv' in [51] concerns the full $\tau$-dependence of certain generating series of eMZVs or the composing iterated Eisenstein integrals. This reference associates open-string prototypes (i.e. esv preimages) to the simplest closed-string integrals at genus one whose integrands are solely built from Green functions involving any number of punctures. On the one hand, the proposal for the single-valued map 


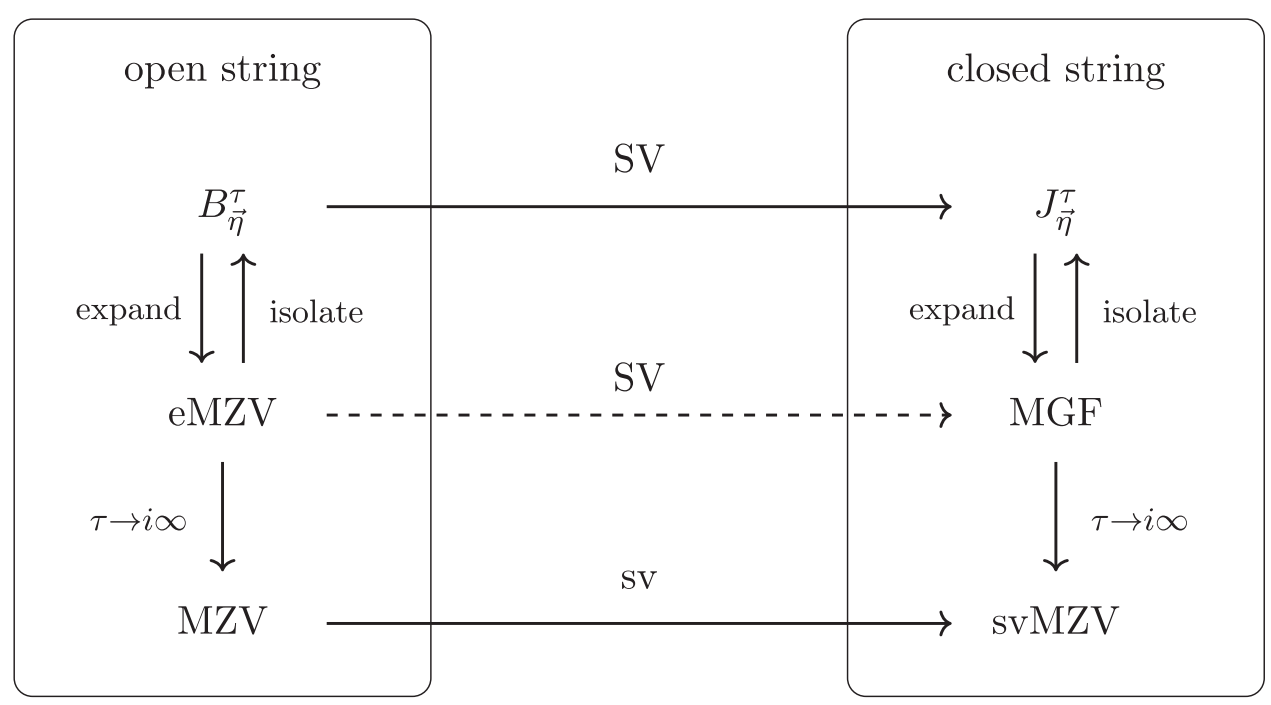

Figure 1. Diagram illustrating the various pieces involved in constructing the proposal SV for an elliptic single-valued map with open-string quantities on its left-hand side and closed-string quantities on its right-hand side. The generating series $B_{\vec{\eta}}^{\tau}$ of the open string contains eMZVs in its $\alpha^{\prime}$ - and $\eta_{j}$-expansion. Conversely, a given eMZV can be isolated as a specific component of the generating series. The $\tau$-dependent eMZVs contain MZVs in their degeneration limit $\tau \rightarrow i \infty$. Similarly, the closed-string generating series $J_{\vec{\eta}}^{\tau}$ yields MGFs upon expansion and MGFs can be isolated as specific components in this expansion. The degeneration limit $\tau \rightarrow i \infty$ of MGFs is expected to only contain single-valued multiple zeta values that are related to the MZV by the known singlevalued map sv. Instead of attempting a direct construction of an elliptic SV-map from eMZVs to MGFs, we exploit the differential equations of the generating series $B_{\vec{\eta}}^{\tau}$ and $J_{\vec{\eta}}^{\tau}$ together with their boundary values from $\tau \rightarrow i \infty$ to describe the map SV at the level of generating series, see (1.1). From this one can extract the map SV: eMZV $\rightarrow$ MGF by inspecting individual orders in the $\alpha^{\prime}$ - and $\eta_{j}$-expansions.

in [51] is contained in (1.1) upon symmetrizing over the integration cycles on its left-hand side and extracting the lowest order in $\eta_{j}$. On the other hand, the implementation of the singlevalued map at the level of iterated Eisenstein integrals in the reference is very different from the proposal in the present work. In comparison to the proposal of [51], our SV action on iterated Eisenstein integrals in (4.30) does not necessarily generate real combinations and is therefore applicable to imaginary cusp forms and MGFs of different holomorphic and antiholomorphic modular weights. Moreover, in contrast to esv in [51], the SV map in (1.1) is observed to be compatible with shuffle multiplication in all known examples. Our SV map additionally introduces combinations of (conjecturally single-valued) MZVs and antiholomorphic terms, which are absent in [51].

At the time of writing, the antiholomorphic admixtures introduced by our SV map on iterated Eisenstein integrals at depth $\geqslant 2$ can only be fixed by indirect methods beyond the reach of open-string data. Instead, the explicit form of the SV action on iterated Eisenstein integrals has so far been extracted from the reality properties of closed-string generating series in [18] that extend the $J_{\vec{\eta}}^{\tau}$ series as described below. However, this limitation does not affect the formulation of our SV map at the level of the lattice-sum representation of MGFs: by virtue of the antielliptic functions $\overline{V(\ldots \mid \tau)}$ in (3.8), the SV image of an arbitrary convergent eMZV 
given in (5.39) can be straightforwardly expressed in terms of lattice sums using the integration techniques of $[8-10,52]$ and, for certain weights, further simplified using the Mathematica package [62].

\subsection{Outline}

This work is organized as follows. We start by reviewing open- and closed-string integrals at genus zero and genus one as well as the basic definitions of single-valued MZVs, eMZVs and MGFs in section 2. Then, section 3 is dedicated to the modified generating series of openand closed-string integrals as well as their relation through our proposed single-valued map at genus one. In particular, the central antielliptic integrands and the resulting proposal for an elliptic single-valued map can be found in sections 3.2 and 3.5, respectively. In section 4, we set the stage for generating explicit examples of single-valued eMZVs by introducing a new expansion method for open-string integrals over B-cycles and relating it to similar closedstring $\alpha^{\prime}$-expansions. This leads to the identifications of MGFs as single-valued eMZVs in section 5, where examples of the antielliptic integrands are related to earlier approaches to an elliptic single-valued map in the literature. The resulting lattice-sum representations of all single-valued convergent eMZVs are discussed in section 5.7. In the concluding section 6, we comment on the relation of string amplitudes to the generating series of this work and further directions.

\section{Review of genus-zero and genus-one integrals}

In this section, we collect background material on world-sheet integrals at genus zero and one, including the genus-zero single-valued map, and review various definitions relevant to the single-valued map at genus one.

\subsection{Genus-zero integrals}

We briefly review the basic disk (open-string) and sphere (closed-string) integrals for genuszero world-sheets and how they are related by the genus-zero single-valued map.

2.1.1. Definitions of disk and sphere integrals. Massless tree-level $n$-point amplitudes of the open superstring [63] and the open bosonic string [64] can be expanded in a basis of iterated integrals [65]

$$
Z^{\text {tree }}(\gamma \mid \rho)=\int_{\mathfrak{D}(\gamma)} \frac{\left(\prod_{j=1}^{n} \mathrm{~d} z_{j}\right)}{\operatorname{vol~SL} 2(\mathbb{R})} \prod_{1 \leqslant i<j}^{n}\left|z_{i j}\right|^{-s_{i j}} \operatorname{PT}(\rho(1,2, \ldots, n))
$$

over the boundary of a disk which we parametrize through the real line

$$
\mathfrak{D}(\gamma)=\left\{z_{j} \in \mathbb{R},-\infty<z_{\gamma(1)}<z_{\gamma(2)}<\cdots<z_{\gamma(n)}<\infty\right\} .
$$

The disk integrands involve dimensionless Mandelstam invariants

$$
s_{i j}=-\frac{\alpha^{\prime}}{2} k_{i} \cdot k_{j}, \quad k_{j}^{2}=0
$$

and Parke-Taylor factors

$$
\operatorname{PT}(\rho(1,2, \ldots, n))=\frac{1}{z_{\rho(1) \rho(2)} z_{\rho(2) \rho(3)} \ldots z_{\rho(n) \rho(1)}}, \quad z_{i j}=z_{i}-z_{j} .
$$


The inverse vol $\mathrm{SL}_{2}(\mathbb{R})$ in $(2.1)$ instructs us to set any triplet of punctures to $0,1, \infty$, where the $\mathrm{SL}_{2}(\mathbb{R})$ invariance of genus-zero integrands hinges on momentum conservation $\sum_{j=1}^{n} k_{j}=0$. Both the domains and the Parke-Taylor integrands are indexed via permutations $\gamma, \rho \in S_{n}$ of the external legs $1,2, \ldots, n$. One can arrive at smaller bases of $(n-3)$ ! cycles $\gamma$ and Parke-Taylor orderings $\rho$ via monodromy relations $[66,67]$ and integration by parts $[63,65]$, respectively.

Closed-string tree amplitudes in turn can be reduced to sphere integrals

$$
\begin{aligned}
& J^{\text {tree }}(\gamma \mid \rho)=\frac{1}{\pi^{n-3}} \int_{\mathbb{C}^{n-3}} \frac{\left(\prod_{j=1}^{n} \mathrm{~d}^{2} z_{j}\right)}{\operatorname{vol~SL}(\mathbb{C})} \\
& \times \prod_{1 \leqslant i<j}^{n}\left|z_{i j}\right|^{-2 s_{i j}} \overline{\mathrm{PT}(\gamma(1,2, \ldots, n))} \mathrm{PT}(\rho(1,2, \ldots, n))
\end{aligned}
$$

involving $\mathrm{d}^{2} z_{j}=\frac{i}{2} \mathrm{~d} z_{j} \wedge \mathrm{d} \bar{z}_{j}$ and permutations $\gamma, \rho \in S_{n}$ of meromorphic and antimeromorphic Parke-Taylor factors subject to the same integration-by-parts relations as in the open-string case.

2.1.2. Single-valued map between disk and sphere integrals. The disk and sphere integrals (2.1) and (2.5) converge for a suitable range of the $\operatorname{Re}\left(s_{i j}\right)$ and they admit a Laurent expansion in $\alpha^{\prime}$, i.e. around the value $s_{i j}=0$ of the dimensionless Mandelstam invariants (2.3). The coefficients in the $\alpha^{\prime}$-expansions of disk integrals $Z^{\text {tree }}$ are MZVs [68, 69],

$$
\zeta_{n_{1}, n_{2}, \ldots, n_{r}}=\sum_{0<k_{1}<k_{2}<\cdots<k_{r}} k_{1}^{-n_{1}} k_{2}^{-n_{2}} \ldots k_{r}^{-n_{r}}, \quad n_{r} \geqslant 2
$$

whose weight $n_{1}+n_{2}+\cdots+n_{r}$ matches the order in $\alpha^{\prime}$ beyond the low-energy limit (i.e. beyond the leading order in $\alpha^{\prime}$ ). The polynomial structure of the $Z^{\text {tree }}$ in $s_{i j}$ can for instance be generated from the Drinfeld associator [70] or Berends-Giele recursions [71], with explicit results available for download from [72, 73].

When applying the single-valued map [46, 48] of motivic [74] $\mathrm{MZVs}^{8}$

$$
\mathrm{sv} \zeta_{2 k}=0, \quad \mathrm{sv} \zeta_{2 k+1}=2 \zeta_{2 k+1}, \quad \mathrm{sv} \zeta_{3,5}=-10 \zeta_{3} \zeta_{5}, \quad \text { etc }
$$

order by order in $\alpha^{\prime}$, the disk and sphere integrals (2.1) and (2.5) are related by [40-45]

$$
J^{\text {tree }}(\gamma \mid \rho)=\operatorname{sv} Z^{\text {tree }}(\gamma \mid \rho)
$$

The first permutation $\gamma$ in $Z^{\text {tree }}$ and $J^{\text {tree }}$ refers to a disk ordering (2.2) and an antimeromorphic Parke-Taylor factor (2.4), respectively, which are connected by a Betti-deRham duality $[49,57,58]$. The key result of this work is to identify similar pairs of cycles and antimeromorphic functions at genus one.

\subsection{Genus-one integrals}

As a preparation for our proposal of a genus-one single-valued map, we now introduce the basic genus-one world-sheet integrals and the objects appearing in their $\alpha^{\prime}$-expansion.

\footnotetext{
${ }^{8}$ Strictly speaking, MZVs need to be replaced by their motivic versions to have a well-defined single-valued map.
} 

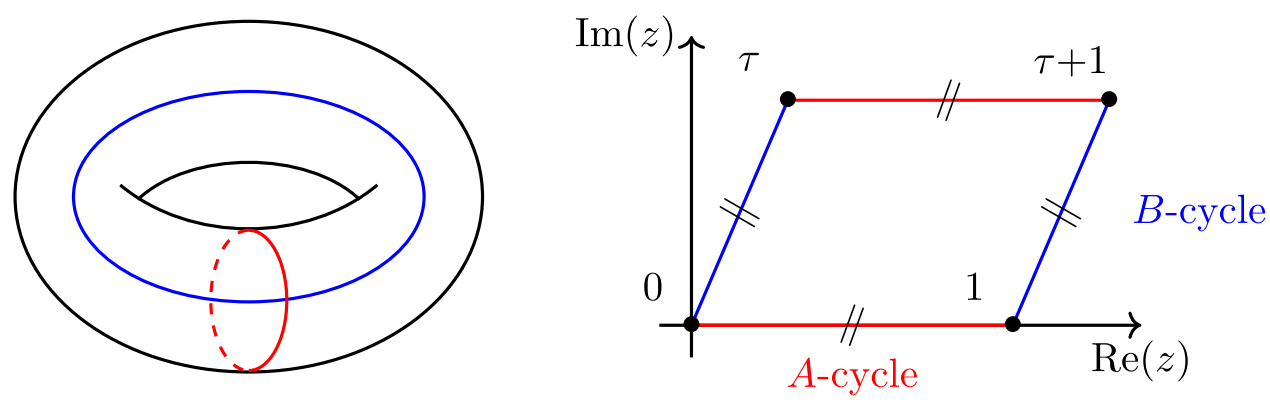

Figure 2. Parametrization of the torus $\mathfrak{T}=\frac{\mathbb{C}}{\mathbb{Z}+\tau \mathbb{Z}}$ with identifications $z \cong z+1 \cong z+\tau$ marked by $\|$ along the A- and B-cycles. While the torus is drawn for non-vanishing $\operatorname{Re}(\tau)$ to accommodate closed-string amplitudes, the cylinder world-sheets for openstring amplitudes are derived from tori at $\tau \in i \mathbb{R}^{+}$via suitable involutions [75].

2.2.1. Genus-one open-string A-cycle integrals. In the same way as disk integrals can be cast into a Parke-Taylor-type basis (2.1), the basis integrals for massless genus-one open-string amplitudes are claimed to be generated by $[59,60]$

$$
Z_{\vec{\eta}}^{\tau}(\gamma \mid \rho)=\int_{\mathfrak{A}(\gamma)}\left(\prod_{j=2}^{n} \mathrm{~d} z_{j}\right) \varphi_{\vec{\eta}}^{\tau}(1, \rho(2, \ldots, n)) \prod_{1 \leqslant i<j}^{n} \mathrm{e}^{s_{i j} \mathcal{G}_{\mathfrak{A}}\left(z_{i j}, \tau\right)}
$$

where we have set $z_{1}=0$ by translation invariance. In this work we restrict to planar amplitudes with all state insertions on a single cylinder boundary (as opposed to non-planar amplitudes with punctures on both boundaries of the cylinder). We do not impose momentum conservation in a genus-one context and treat all the $s_{i j}$ with $1 \leqslant i<j \leqslant n$ as independent. The ordering of the open-string punctures on a cylinder boundary is encoded in an integration domain on the A-cycle of a torus (see figure 2 for the standard parametrization) with $\tau \in i \mathbb{R}^{+}[75]$

$$
\mathfrak{A}(\gamma)=\left\{z_{j} \in \mathbb{R}, 0<z_{\gamma(2)}<z_{\gamma(3)}<\cdots<z_{\gamma(n)}<1\right\}
$$

with similar integration domains [76] for the non-planar open-string integrals.

The integrand of (2.9) features the open-string Green function on an A-cycle (which is chosen to enforce $\mathcal{G}_{\mathfrak{A}}(z, \tau)=\mathcal{G}_{\mathfrak{A}}(-z, \tau)$ and $\left.\int_{0}^{1} \mathrm{~d} z \mathcal{G}_{\mathfrak{A}}(z, \tau)=0[51,77]\right)$

$$
\mathcal{G}_{\mathfrak{A}}(z, \tau)=-\log \left(\frac{\theta_{1}(|z|, \tau)}{\eta(\tau)}\right)+\frac{i \pi \tau}{6}+\frac{i \pi}{2}, \quad z \in(-1,1)
$$

and the following combination of the doubly-periodic Kronecker-Eisenstein series [78]

$$
\begin{gathered}
\Omega(z, \eta, \tau)=\exp \left(2 \pi i \eta \frac{\operatorname{Im} z}{\operatorname{Im} \tau}\right) \frac{\theta_{1}^{\prime}(0, \tau) \theta_{1}(z+\eta, \tau)}{\theta_{1}(z, \tau) \theta_{1}(\eta, \tau)} \\
\varphi_{\vec{\eta}}^{\tau}(1,2, \ldots, n)=\Omega\left(z_{12}, \eta_{23 \ldots n}, \tau\right) \Omega\left(z_{23}, \eta_{3 \ldots n}, \tau\right) \cdots \Omega\left(z_{n-1, n}, \eta_{n}, \tau\right)
\end{gathered}
$$


with $\eta_{i j \ldots k}=\eta_{i}+\eta_{j}+\cdots+\eta_{k} \cdot{ }^{9}$ The permutation $\rho \in S_{n-1}$ in $\varphi_{\vec{\eta}}^{\tau}(1, \rho(2, \ldots, n))$ is taken to act on both the $z_{j}$ and the formal expansion variables $\eta_{j} \in \mathbb{C}$ in (2.13). The conjectural basis (2.9) is a generating function of the world-sheet integrals over the Kronecker-Eisenstein coefficients $f^{(w)}$

$$
\Omega(z, \eta, \tau)=\sum_{w=0}^{\infty} \eta^{w-1} f^{(w)}(z, \tau)
$$

that occur in the integrands of genus-one open- and closed-string amplitudes [4, 52, 79], e.g.

$$
f^{(0)}(z, \tau)=1, \quad f^{(1)}(z, \tau)=\partial_{z} \log \theta_{1}(z, \tau)+2 \pi i \frac{\operatorname{Im} z}{\operatorname{Im} \tau} .
$$

While the massless four-point genus-one amplitude of the open superstring [80] is proportional to the most singular $\eta_{j}^{-3}$-order of $Z_{\vec{\eta}}^{\tau}(\cdot \mid 1,2,3,4)$, the analogous amplitude of the open bosonic string additionally involves contributions of $Z_{\vec{\eta}}^{\tau}(\cdot \mid 1,2,3,4)$ (and its permutations in 2,3,4) at the orders of $\eta_{j}^{ \pm 1}$ [76]. ${ }^{10}$ The short-distance behavior $f^{(1)}(z, \tau)=\frac{1}{z}+\mathcal{O}(z)$ introduces kinematic poles into the $\alpha^{\prime}$-expansion of (2.9), and the remaining $f^{(w \neq 1)}(z, \tau)$ are regular for any $z \in \mathbb{C}$.

2.2.2. Genus-one closed-string integrals. In the same way as (2.9) is claimed to be a universal basis of genus-one open-string integrals, the integrals over the torus punctures for massless genus-one amplitudes in type II, heterotic and bosonic string theories should be generated by [61]

$$
\begin{aligned}
Y_{\vec{\eta}}^{\tau}(\gamma \mid \rho)= & (2 i)^{n-1} \int_{\mathfrak{T}^{n-1}}\left(\prod_{j=2}^{n} \mathrm{~d}^{2} z_{j}\right) \prod_{1 \leqslant i<j}^{n} \mathrm{e}^{s_{i j} \mathcal{G}_{\mathfrak{T}}\left(z_{i j}, \tau\right)} \frac{\varphi_{\vec{\eta}}^{\tau}(1, \gamma(2, \ldots, n))}{} \\
& \times \varphi_{(\tau-\bar{\tau}) \vec{\eta}}^{\tau}(1, \rho(2, \ldots, n))
\end{aligned}
$$

with $z_{1}=0$. The remaining $z_{j}$ are integrated over the torus $\mathfrak{T}=\frac{\mathbb{C}}{\tau \mathbb{Z}+\mathbb{Z}}$ with modular parameter $\tau \in \mathbb{H}=\{\tau \in \mathbb{C}, \operatorname{Im} \tau>0\}$. The closed-string Green function

$$
\mathcal{G}_{\mathfrak{T}}(z, \tau)=-\log \left|\frac{\theta_{1}(z, \tau)}{\eta(\tau)}\right|^{2}+\frac{2 \pi(\operatorname{Im} z)^{2}}{\operatorname{Im} \tau}
$$

is chosen to be modular invariant and to obey $\int_{\mathfrak{T}} \mathrm{d}^{2} z \mathcal{G}_{\mathfrak{T}}(z, \tau)=0$, and its holomorphic derivatives parallel those of the open-string Green function $\mathcal{G}_{\mathfrak{A}}(z, \tau)$ in $(2.11)$,

$$
\begin{aligned}
\partial_{z} \mathcal{G}_{\mathfrak{T}}(z, \tau) & =-f^{(1)}(z, \tau), & 2 \pi i \partial_{\tau} \mathcal{G}_{\mathfrak{T}}(u \tau+v, \tau) & =-f^{(2)}(u \tau+v, \tau) \\
\partial_{v} \mathcal{G}_{\mathfrak{A}}(v, \tau) & =-f^{(1)}(v, \tau), & 2 \pi i \partial_{\tau} \mathcal{G}_{\mathfrak{A}}(v, \tau) & =-f^{(2)}(v, \tau)-2 \zeta_{2},
\end{aligned}
$$

${ }^{9}$ Our conventions for the standard odd Jacobi theta function are

$$
\theta_{1}(z, \tau)=q^{1 / 8}\left(\mathrm{e}^{i \pi z}-\mathrm{e}^{-i \pi z}\right) \prod_{n=1}^{\infty}\left(1-q^{n}\right)\left(1-\mathrm{e}^{2 \pi i z} q^{n}\right)\left(1-\mathrm{e}^{-2 \pi i z} q^{n}\right)
$$

and $\eta(\tau)$ is the Dedekind eta function. In order to avoid confusion with the expansion parameters $\eta_{j}$, we always spell out the argument $\tau$ of the Dedekind eta function. Representations of the open-string Green function in terms of elliptic polylogarithms are discussed in [4, 5, 51], and we follow the conventions of [4] for regularizing endpoint divergences.

${ }^{10}$ By using Fay identities and integration by parts, the massless four-point genus-one amplitude of open bosonic strings in section 8.1.1 of [76] can be rewritten in terms of the coefficients in the $\eta_{j}$-expansion of $Z_{\vec{\eta}}^{\tau}(\cdot \mid 1,2,3,4)$. 
where $u, v \in \mathbb{R}$ parametrize the covering space of the torus and the $f^{(w)}(z, \tau)$ with $z=u \tau+v$ are defined by (2.15). The second arguments $(\tau-\bar{\tau}) \eta_{j}$ and $\bar{\eta}_{j}$ of the Kronecker-Eisenstein series and their complex conjugates in (2.16) have been chosen such that each order in the $\eta_{j}$ and $\alpha^{\prime}$-expansion gives rise to modular forms of purely antiholomorphic modular weight ${ }^{11}$.

When assembling genus-one amplitudes of open and closed strings from the series $Z_{\vec{\eta}}^{\tau}$ and $Y_{\vec{\eta}}^{\tau}$, it remains to dress the component integrals in their $\eta_{j}$-expansions with kinematic factors that carry the dependence on the external polarizations. The latter are determined from the conformal-field-theory correlators of the vertex operators, see e.g. [81, 82], and are unaffected by our proposal for the single-valued map at genus one.

2.2.3. Differential equations in $\tau$. Based on the differential equations (2.18) of the Green functions and integration by parts in the $z_{j}$, the open- and closed-string integrals (2.9) and (2.16) were shown in $[60,61]$ to obey the differential equations

$$
\begin{aligned}
& 2 \pi i \partial_{\tau} Z_{\vec{\eta}}^{\tau}(\gamma \mid \rho)=\sum_{k=0}^{\infty}(1-k) G_{k}(\tau) \sum_{\alpha \in S_{n-1}} r_{\vec{\eta}}\left(\epsilon_{k}\right)_{\rho}^{\alpha} Z_{\vec{\eta}}^{\tau}(\gamma \mid \alpha) \\
& 2 \pi i \partial_{\tau} Y_{\vec{\eta}}^{\tau}(\gamma \mid \rho)=\sum_{k=0}^{\infty}(1-k)(\tau-\bar{\tau})^{k-2} G_{k}(\tau) \sum_{\alpha \in S_{n-1}} R_{\vec{\eta}}\left(\epsilon_{k}\right)_{\rho}{ }^{\alpha} Y_{\vec{\eta}}^{\tau}(\gamma \mid \alpha),
\end{aligned}
$$

respectively. The right-hand sides involve holomorphic Eisenstein series $G_{0}=-1$ and

$$
G_{k}(\tau)=\sum_{\substack{m, n \in \mathbb{Z} \\(m, n) \neq(0,0)}} \frac{1}{(m \tau+n)^{k}}, \quad k \geqslant 4
$$

as well as $(n-1) ! \times(n-1)$ ! matrices $r_{\vec{\eta}}\left(\epsilon_{k}\right), R_{\vec{\eta}}\left(\epsilon_{k}\right)$ independent of $\tau$ that vanish for $k=2$ and $k \in 2 \mathbb{N}-1$. This means in particular that $G_{2}(\tau)$ does not appear in (2.19).

The two-point instances are

$$
\begin{aligned}
r_{\eta_{2}}\left(\epsilon_{0}\right) & =s_{12}\left(\frac{1}{\eta_{2}^{2}}+2 \zeta_{2}-\frac{1}{2} \partial_{\eta_{2}}^{2}\right) \\
r_{\eta_{2}}\left(\epsilon_{k}\right) & =R_{\eta_{2}}\left(\epsilon_{k}\right)=s_{12} \eta_{2}^{k-2}, \quad k \geqslant 4 \\
R_{\eta_{2}}\left(\epsilon_{0}\right) & =s_{12}\left(\frac{1}{\eta_{2}^{2}}-\frac{1}{2} \partial_{\eta_{2}}^{2}\right)-2 \pi i \bar{\eta}_{2} \partial_{\eta_{2}} .
\end{aligned}
$$

The notation $\epsilon_{k}$ reflects the expectation that the $r_{\vec{\eta}}\left(\epsilon_{k}\right), R_{\vec{\eta}}\left(\epsilon_{k}\right)$ are matrix representations of Tsunogai's derivation algebra [83] and obey relations such as (see [17, 84, 85] for similar relations at higher weight and depth)

$$
\left[r_{\vec{\eta}}\left(\epsilon_{10}\right), r_{\vec{\eta}}\left(\epsilon_{4}\right)\right]-3\left[r_{\vec{\eta}}\left(\epsilon_{8}\right), r_{\vec{\eta}}\left(\epsilon_{6}\right)\right]=0 .
$$

The all-multiplicity formulae for these $(n-1) ! \times(n-1) !$ representations in [60, 61] manifest that the $r_{\vec{\eta}}\left(\epsilon_{k}\right)$ are linear in the $s_{i j}$, i.e. proportional to $\alpha^{\prime}$, and their closed-string analogues

\footnotetext{
${ }^{11}$ Functions $F(\tau)$ on the upper half plane with transformations $F\left(\frac{\alpha \tau+\beta}{\gamma \tau+\delta}\right)=(\gamma \tau+\delta)^{w}(\gamma \bar{\tau}+\delta)^{\bar{w}} F(\tau)$ under $\left(\begin{array}{ll}\alpha & \beta \\ \gamma & \delta\end{array}\right) \in$ $\mathrm{SL}_{2}(\mathbb{Z})$ are said to carry holomorphic and antiholomorphic modular weight $w$ and $\bar{w}$, respectively.
} 
$R_{\vec{\eta}}\left(\epsilon_{k}\right)$ additionally involve terms $\sim \bar{\eta}_{j} \partial_{\eta_{j}}$ independent of $\alpha^{\prime}$ (with $s_{12 \ldots n}=\sum_{1 \leqslant i<j}^{n} s_{i j}$ ):

$$
R_{\vec{\eta}}\left(\epsilon_{k}\right)=\left\{\begin{array}{ll}
r_{\vec{\eta}}\left(\epsilon_{k}\right) & : k \geqslant 4 \\
r_{\vec{\eta}}\left(\epsilon_{0}\right)-2 \zeta_{2} s_{12 \ldots n}-2 \pi i \sum_{j=2}^{n} \bar{\eta}_{j} \partial_{\eta_{j}} & : k=0
\end{array} .\right.
$$

2.2.4. Basic definitions of eMZVs and MGFs. We shall now review the definitions of the eMZVs and MGFs that occur as the expansion coefficients of the above genus-one integrals. The $\eta_{j}$ - and $\alpha^{\prime}$-expansion of the open-string integrals $Z_{\vec{\eta}}^{\tau}(\gamma \mid \rho)$ in (2.9) gives rise to A-cycle eMZVs [4-6]

$$
\omega\left(n_{1}, n_{2}, \ldots, n_{r} \mid \tau\right)=\int_{0<z_{1}<z_{2}<\cdots<z_{r}<1} \mathrm{~d} z_{1} f^{\left(n_{1}\right)}\left(z_{1}, \tau\right) \mathrm{d} z_{2} f^{\left(n_{2}\right)}\left(z_{2}, \tau\right) \ldots \mathrm{d} z_{r} f^{\left(n_{r}\right)}\left(z_{r}, \tau\right)
$$

introduced by Enriquez [3] which are said to carry weight $n_{1}+n_{2}+\cdots+n_{r}$ and length $r$. Endpoint divergences in case of $n_{1}=1$ or $n_{r}=1$ are shuffle-regularized as in section 2.2.1 of [4]. The specific eMZVs at a given order of $Z_{\vec{\eta}}^{\tau}(\gamma \mid \rho)$ in $s_{i j}$ and $\eta_{j}$ can be obtained from the differential equations (2.19) along with the initial values $Z_{\vec{\eta}}^{\tau \rightarrow i \infty}(\gamma \mid \rho)$ in [60] or from matrix representations of the elliptic KZB associator [86, 87].

The closed-string integrals $Y_{\vec{\eta}}^{\tau}(\gamma \mid \rho)$ in (2.16) in turn introduce multiple sums over the momentum lattice of a torus $[52,61]$

$$
\Lambda=\mathbb{Z}+\tau \mathbb{Z}, \quad \Lambda^{\prime}=\Lambda \backslash\{0\}
$$

that are known as MGFs $[10,11]$. With the removal of $p=0$ from $\Lambda$, they can be thought of as infrared-regulated and discretized versions of Feynman integrals on a torus. The MGFs associated with Feynman graphs of dihedral topology are defined by ${ }^{12}$

$$
\mathcal{C}\left[\begin{array}{llll}
a_{1} & a_{2} & \ldots & a_{r} \\
b_{1} & b_{2} & \ldots & b_{r}
\end{array}\right]=\sum_{p_{1}, p_{2}, \ldots, p_{r} \in \Lambda^{\prime}} \frac{\delta\left(p_{1}+p_{2}+\cdots+p_{r}\right)}{p_{1}^{a_{1}} \bar{p}_{1}^{b_{1}} p_{2}^{a_{2}} \bar{p}_{2}^{b_{2}} \ldots p_{r}^{a_{r}} \bar{p}_{r}^{b_{r}}}
$$

and more general topologies are for instance discussed in [11, 62]. The simplest examples of dihedral MGFs (2.26) have two columns and are associated with one-loop graphs on the world-sheet

$$
\mathcal{C}\left[\begin{array}{ll}
a & 0 \\
b & 0
\end{array}\right]=\sum_{p \in \Lambda^{\prime}} \frac{1}{p^{a} \bar{p}^{b}}
$$

whereas $\mathcal{C}\left[\begin{array}{llll}a_{1} & a_{2} & \ldots & a_{r} \\ b_{1} & b_{2} & \ldots & b_{r}\end{array}\right]$ are referred to as $(r-1)$-loop MGFs. As long as the entries obey $a+b>2$, the lattice sums (2.27) are absolutely convergent and the one-loop MGFs are expressible in terms of non-holomorphic Eisenstein series $E_{k}(\tau)$ and their Cauchy-Riemann derivatives

\footnotetext{
${ }^{12}$ Note that the definition of $\mathcal{C}[\ldots]$ in this work follows the conventions of $[18,61,62]$ but differs from those in [11, $52,54,88]$ by factors of $\operatorname{Im} \tau$ and $\pi$.
} 


$$
\begin{aligned}
E_{k}(\tau)=\left(\frac{\operatorname{Im} \tau}{\pi}\right)^{k} \mathcal{C}\left[\begin{array}{ll}
k & 0 \\
k & 0
\end{array}\right], & \nabla^{m} E_{k}(\tau)=\frac{(\operatorname{Im} \tau)^{k+m}}{\pi^{k}} \frac{(k+m-1) !}{(k-1) !} \mathcal{C}\left[\begin{array}{ll}
k+m & 0 \\
k-m & 0
\end{array}\right], \\
\bar{\nabla}^{m} E_{k}(\tau) & =\frac{(\operatorname{Im} \tau)^{k+m}}{\pi^{k}} \frac{(k+m-1) !}{(k-1) !} \mathcal{C}\left[\begin{array}{ll}
k-m & 0 \\
k+m & 0
\end{array}\right],
\end{aligned}
$$

where $\nabla=2 i(\operatorname{Im} \tau)^{2} \partial_{\tau}$ and $\bar{\nabla}=-2 i(\operatorname{Im} \tau)^{2} \partial_{\bar{\tau}}$. As will be detailed below, both eMZVs (2.24) and MGFs such as (2.26) can be represented via iterated integrals of holomorphic Eisenstein series $G_{k}=\mathcal{C}\left[\begin{array}{ll}k & 0 \\ 0 & 0\end{array}\right]$ defined by (2.20). Both eMZVs [17] and MGFs [11, 62, 88-90] exhibit a multitude of relations over rational combinations of MZVs, all of which are automatically exposed in their iterated-Eisenstein-integral representation ${ }^{13}$. A computer implementation for the decomposition of a large number of eMZVs and MGFs into basis elements is available in $[62,92]$, respectively.

\section{New types of genus-one integrals}

The goal of this paper is to relate the $\alpha^{\prime}$-expansions of suitable generating functions of genusone open- and closed-string integrals. The $Z_{\vec{\eta}}^{\tau}(\gamma \mid \rho)$ and $Y_{\vec{\eta}}^{\tau}(\gamma \mid \rho)$ in (2.9) and (2.16) can be anticipated to not yet furnish the optimal building blocks for this purpose since

(a) The $\tau$-dependence $\sim G_{k}(\tau)$ and $\sim(\tau-\bar{\tau})^{k-2} G_{k}(\tau)$ of the open- and closed-string differential equations (2.19) does not match, even in absence of $\bar{\tau}$.

(b) The contributions $\sim \bar{\eta}_{j} \partial_{\eta_{j}}$ to the closed-string derivations $R_{\vec{\eta}}\left(\epsilon_{0}\right)$ in (2.21) and (2.23) do not have any open-string counterpart in $r_{\bar{\eta}}\left(\epsilon_{0}\right)$.

Both of these shortcomings will be fixed by the improved open- and closed-string generating functions $\boldsymbol{B}_{\bar{\eta}}^{\tau}(\gamma \mid \rho)$ and $\boldsymbol{J}_{\vec{\eta}}^{\tau}(\gamma \mid \rho)$ to be introduced in this section.

\subsection{Genus-one open-string B-cycle integrals}

Instead of parametrizing the cylinder boundary through the A-cycle of a torus as in (2.9), one can perform a modular $S$ transformation

$$
\begin{aligned}
B_{\vec{\eta}}^{\tau}(\gamma \mid \rho) & =Z_{\vec{\eta}}^{-1 / \tau}(\gamma \mid \rho) \\
& =\int_{\mathfrak{B}(\gamma)}\left(\prod_{j=2}^{n} \mathrm{~d} z_{j}\right) \varphi_{\tau \vec{\eta}}^{\tau}(1, \rho(2, \ldots, n)) \prod_{1 \leqslant i<j}^{n} \mathrm{e}^{s_{i j} \mathcal{G}_{\mathfrak{B}}\left(z_{i j}, \tau\right)}
\end{aligned}
$$

to attain a parametrization through the B-cycle (recalling that $z_{1}=0$ and $\tau \in i \mathbb{R}^{+}$)

$$
\begin{aligned}
\mathfrak{B}(\gamma)= & \bigoplus_{j=1}^{n} \mathfrak{B}_{j}(\gamma) \\
\mathfrak{B}_{j}(\gamma)= & \left\{z_{i}=\tau u_{i},-\frac{1}{2}<u_{\gamma(j+1)}<u_{\gamma(j+2)}<\cdots<u_{\gamma(n)}<0<u_{\gamma(2)}\right. \\
& \left.<u_{\gamma(3)}<\cdots<u_{\gamma(j)}<\frac{1}{2}\right\}
\end{aligned}
$$

\footnotetext{
${ }^{13}$ This relies on the linear-independence result of [91] on holomorphic iterated Eisenstein integrals.
} 

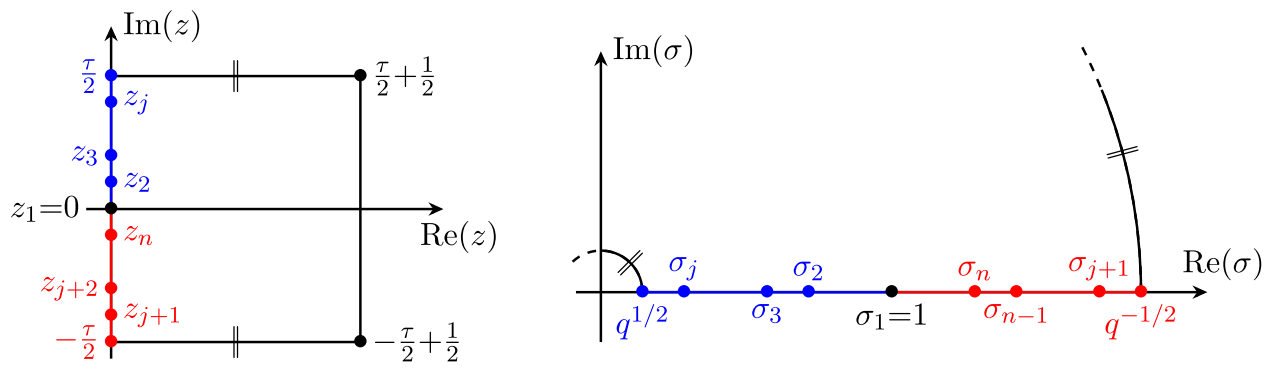

Figure 3. The parametrization (3.2) of the B-cycle is mapped to the positive real axis in the $\sigma_{j}=\mathrm{e}^{2 \pi i z_{j}}$ variables which exhausts all of $\mathbb{R}^{+}$as $\tau \rightarrow i \infty$ and $q=\mathrm{e}^{2 \pi i \tau} \rightarrow 0$. The line segments in the $z$-coordinate and the semicircles in the $\sigma$-coordinate marked by $\|$ are identified by the periodic direction of the cylinder, i.e. the B-cycle of the parental torus.

where $u_{i} \in \mathbb{R}$, and the B-cycle Green function $\mathcal{G}_{\mathfrak{B}}(z, \tau)$ is constructed in two steps: first, we define $\mathcal{G}_{\mathfrak{B}}(z, \tau)$ for $z$ on the line $(0, \tau)$ by [77]

$$
\mathcal{G}_{\mathfrak{B}}(z, \tau)=\mathcal{G}_{\mathfrak{A}}\left(\frac{z}{\tau},-\frac{1}{\tau}\right)=-\frac{i \pi z^{2}}{\tau}-\log \left(\frac{\theta_{1}(z, \tau)}{\eta(\tau)}\right)-\frac{i \pi}{6 \tau}+i \pi, \quad z \in(0, \tau) .
$$

Then, we extend this to $z \in(-\tau, 0)$ by imposing $\mathcal{G}_{\mathfrak{B}}(z, \tau)=\mathcal{G}_{\mathfrak{B}}(-z, \tau)$ for compatibility with (2.11) under modular $S$ transformations, leading to the combined expression

$$
\mathcal{G}_{\mathfrak{B}}(u \tau, \tau)=-i \pi u^{2} \tau-\log \left(\frac{\theta_{1}(|u| \tau, \tau)}{\eta(\tau)}\right)-\frac{i \pi}{6 \tau}+i \pi, \quad u \in(-1,1) .
$$

Instead of integrating over $z_{i}=\tau u_{i}$ with $u_{i} \in(0,1)$, we have chosen the representative $u_{i} \in\left(-\frac{1}{2}, \frac{1}{2}\right)$ of the B-cycle in order to facilitate the comparison with genus-zero integration cycles as $\tau \rightarrow i \infty$. Figure 3 illustrates the integration cycle (3.2) in both the $z_{j}$ and $\sigma_{j}=\mathrm{e}^{2 \pi i z_{j}}$ variables (the latter becoming the coordinates on the sphere as $\tau \rightarrow i \infty$ ), where $z_{j} \in i \mathbb{R}$ and $\sigma_{j} \in \mathbb{R}^{+}$for purely imaginary choices of $\tau$. Note that non-planar versions of the B-cycle integrals involve additional punctures at $z_{j} \in \frac{1}{2}+i \mathbb{R}$ or negative $\sigma_{j} \in\left(-q^{-1 / 2},-q^{1 / 2}\right)$.

The modular transformation $\Omega\left(z, \eta,-\frac{1}{\tau}\right)=\tau \Omega(\tau z, \tau \eta, \tau)$ of the doubly-periodic Kronecker-Eisenstein series (2.12) leads to the rescaling $\eta_{j} \rightarrow \tau \eta_{j}$ in the subscript of the $\rho$-dependent integrand $\varphi_{\tau \vec{\eta}}^{\tau}$ of (3.1).

\subsection{Dual closed-string integrals}

The doubly-periodic integrands $\varphi_{\vec{\eta}}^{\tau}$ in (2.13) are non-holomorphic, so their complex conjugates in (2.16) obey

$$
\partial_{z_{j}} \overline{\varphi_{\vec{\eta}}^{\tau}(1, \gamma(2, \ldots, n))}=\frac{2 \pi i \bar{\eta}_{j}}{\tau-\bar{\tau}} \overline{\varphi_{\vec{\eta}}^{\tau}(1, \gamma(2, \ldots, n))}
$$

which leads to the terms $\sim \bar{\eta}_{j} \partial_{\eta_{j}}$ in the closed-string derivations $R_{\vec{\eta}}\left(\epsilon_{0}\right)$ in (2.23). This introduces a tension between the open- and closed-string differential equation (2.19) such that the $\varphi_{\vec{\eta}}^{\tau}$ do not qualify as Betti-deRham duals of open-string integration cycles. In order to generalize the interplay of Parke-Taylor factors (2.4) with single-valued integration [48, 49] to genus 
one, the factor of $\overline{\varphi_{\vec{\eta}}^{\tau}(\ldots)}$ in the $Y_{\vec{\eta}}^{\tau}$ integrals (2.16) needs to be replaced by an antimeromorphic function that is still well-defined on the torus, i.e. the complex conjugate of an elliptic function in all of $z_{1}, z_{2}, \ldots, z_{n}$.

Such elliptic functions of $n$ punctures can be generated by cycles of Kronecker-Eisenstein series [79]

$$
\Omega\left(z_{12}, \xi, \tau\right) \Omega\left(z_{23}, \xi, \tau\right) \ldots \Omega\left(z_{n 1}, \xi, \tau\right)=: \xi^{-n} \sum_{w=0}^{\infty} \xi^{w} V_{w}(1,2, \ldots, n \mid \tau)
$$

where $V_{w}$ has holomorphic modular weight $w$. Even though the individual KroneckerEisenstein series $\Omega$ are not meromorphic in the $z_{j}$, the $V_{w}$ are elliptic functions since the non-holomorphic phase factors in (2.12) cancel from the cyclic product in (3.6). The simplest examples are

$$
\begin{aligned}
V_{0}(1,2, \ldots, n \mid \tau)= & 1 \\
V_{1}(1,2, \ldots, n \mid \tau)= & \sum_{j=1}^{n} f^{(1)}\left(z_{j}-z_{j+1}, \tau\right) \\
V_{2}(1,2, \ldots, n \mid \tau)= & \sum_{j=1}^{n} f^{(2)}\left(z_{j}-z_{j+1}, \tau\right)+\sum_{1 \leqslant j<k}^{n} f^{(1)}\left(z_{j}-z_{j+1}, \tau\right) \\
& \times f^{(1)}\left(z_{k}-z_{k+1}, \tau\right)
\end{aligned}
$$

with $z_{n+1}=z_{1}$ and Kronecker-Eisenstein coefficients $f^{(w)}$ defined by (2.14), also see (5.2) for the analogous expressions at general $w$.

As will be detailed below, these elliptic functions degenerate to suitable combinations of Parke-Taylor factors when forming the linear combinations

$$
V(1,2, \ldots, n \mid \tau)=\sum_{w=0}^{n-2} \frac{V_{w}(1,2, \ldots, n \mid \tau)}{(2 \pi i)^{w}(n-w-1) !}
$$

such as (see sections 5.4 and 5.5 for detailed discussions of the three- and four-point examples)

$$
\begin{aligned}
V(1,2 \mid \tau) & =1, \quad V(1,2,3 \mid \tau)=\frac{1}{2}+\frac{V_{1}(1,2,3 \mid \tau)}{2 \pi i} \\
V(1,2,3,4 \mid \tau)= & \frac{1}{6}+\frac{1}{2} \frac{V_{1}(1,2,3,4 \mid \tau)}{2 \pi i}+\frac{V_{2}(1,2,3,4 \mid \tau)}{(2 \pi i)^{2}} \\
V(1,2,3,4,5 \mid \tau)= & \frac{1}{24}+\frac{1}{6} \frac{V_{1}(1,2,3,4,5 \mid \tau)}{2 \pi i}+\frac{1}{2} \frac{V_{2}(1,2,3,4,5 \mid \tau)}{(2 \pi i)^{2}} \\
& +\frac{V_{3}(1,2,3,4,5 \mid \tau)}{(2 \pi i)^{3}} .
\end{aligned}
$$

To lend credence to this definition of the $V$-function, let us see how their properties parallel those of the genus-zero case: the Betti-deRham duality at genus zero relies on the simple-pole residues

$$
\operatorname{Res}_{z_{j}=z_{j \pm 1}} \mathrm{PT}(1,2, \ldots, j, \ldots, n)= \pm \mathrm{PT}(1,2, \ldots, j-1, j+1, \ldots, n)
$$


of the Parke-Taylor factors (2.4). These residues correspond to the situation when two neighboring points of the disk ordering (2.2) at $z_{j}=z_{j \pm 1}$ come together, which is crucial for sphere integrals being single-valued disk integrals [43, 45].

Similarly, at genus one, the generating function (3.6) of the elliptic $V_{w}$ functions exposes the recursive structure of their simple-pole residues

$$
\operatorname{Res}_{z_{j}=z_{j \pm 1}} V_{w}(1,2, \ldots, j, \ldots, n)= \pm V_{w-1}(1,2, \ldots, j-1, j+1, \ldots, n)
$$

and the absence of higher poles in $z_{j}-z_{j \pm 1}$. Consequently, the pole structure of the elliptic combinations (3.8)

$$
\operatorname{Res}_{z_{j}=z_{j \pm 1}} V(1,2, \ldots, j, \ldots, n)= \pm \frac{1}{2 \pi i} V(1,2, \ldots, j-1, j+1, \ldots, n)
$$

mirrors the boundaries of the open-string integration cycles as $z_{j}=z_{j \pm 1}$, i.e. one recovers mutually consistent $V$-functions and cycles at multiplicity $n-1$ in both cases ${ }^{14}$.

The absence of $V_{w}$ with $w \geqslant n-1$ in (3.8) can be understood from

- The vanishing of $V_{n-1}(1,2, \ldots, n \mid \tau)$ since (3.6) would otherwise be an elliptic function of $\xi$ with a simple pole at the origin [79]

- The breakdown of uniform transcendentality when expanding Koba-Nielsen integrals involving $V_{n}(1,2, \ldots, n \mid \tau)$ [52] (which is in tension with the transcendentality properties of open-string integrals [60])

- The fact that $V_{w \geqslant n+1}(1,2, \ldots, n \mid \tau)$ is expressible in terms of $G_{w-k} V_{k}(1,2, \ldots, n \mid \tau)$ with $k \leqslant n-2[79]$

Similar to the closed-string integrals $Y_{\vec{\eta}}^{\tau}$, we define an $(n-1) ! \times(n-1) !$ matrix of torus integrals

$$
\begin{aligned}
J_{\vec{\eta}}^{\tau}(\gamma \mid \rho)= & (2 i)^{n-1} \int_{\mathfrak{T}^{n-1}}\left(\prod_{j=2}^{n} \mathrm{~d}^{2} z_{j}\right) \prod_{1 \leqslant i<j}^{n} \mathrm{e}^{s_{i j} \mathcal{G}_{\mathfrak{T}}\left(z_{i j}, \tau\right)} \overline{V(1, \gamma(2, \ldots, n))} \\
& \times \varphi_{(\tau-\bar{\tau}) \vec{\eta}}^{\tau}(1, \rho(2, \ldots, n))
\end{aligned}
$$

indexed by permutations $\gamma, \rho \in S_{n-1}$ of (3.8) and (2.13). Note that the cyclic symmetry

$$
V_{w}(2, \ldots, n, 1 \mid \tau)=V_{w}(1,2, \ldots, n \mid \tau), \quad V(2, \ldots, n, 1 \mid \tau)=V(1,2, \ldots, n \mid \tau)
$$

exposed by the generating function (3.6) has been used to bring the integrand of (3.13) into the form of $\overline{V(1, \ldots \mid \tau)}$.

\subsection{Asymptotics at the cusp}

The modular $S$ transformation in (3.1) maps the A-cycle eMZVs (2.24) in the $\eta_{j}$ - and $\alpha^{\prime}$ expansion of $Z_{\vec{\eta}}^{\tau}$ to B-cycle eMZVs [3] in the analogous expansion of $B_{\vec{\eta}}^{\tau}$. As detailed in $[51,77,93]$, the asymptotic expansion of B-cycle eMZVs as $\tau \rightarrow i \infty$ is governed by Laurent polynomials in $T=\pi \tau \in i \mathbb{R}^{+}$whose coefficients are $\mathbb{Q}$-linear combinations of MZVs,

\footnotetext{
${ }^{14}$ On the closed-string side of the 'genus-one Betti-deRham duality' we note that, by double-periodicity of the $V$ functions, additional poles with identical residues occur as $z_{j} \rightarrow z_{j \pm 1}+m \tau+n(m, n, \in \mathbb{Z})$. On the open-string side in turn, the delimiters of the integration cycles in the B-cycle parametrization of figure 3 are separated by $\tau$.
} 
for instance

$$
\begin{gathered}
\omega\left(0,0,2 \mid-\frac{1}{\tau}\right)=-\frac{T^{2}}{180}-\frac{\zeta_{2}}{2}+\frac{i \zeta_{3}}{T}+\frac{3 \zeta_{4}}{2 T^{2}}+\mathcal{O}\left(\mathrm{e}^{2 i T}\right) \\
\omega\left(0,0,1,0 \mid-\frac{1}{\tau}\right)=\frac{i T}{120}-\frac{i \zeta_{2}}{4 T}-\frac{3 \zeta_{3}}{4 T^{2}}+\frac{3 i \zeta_{4}}{4 T^{3}}+\mathcal{O}\left(\mathrm{e}^{2 i T}\right) \\
\omega\left(0,0,3,0 \mid-\frac{1}{\tau}\right)=\frac{i T^{3}}{1260}-\frac{3 i \zeta_{4}}{4 T}-\frac{9 \zeta_{5}}{2 T^{2}}+\frac{15 i \zeta_{6}}{2 T^{3}}+\mathcal{O}\left(\mathrm{e}^{2 i T}\right) .
\end{gathered}
$$

The suppressed terms $\mathcal{O}\left(\mathrm{e}^{2 i T}\right)$ are series in $q=\mathrm{e}^{2 \pi i \tau}=\mathrm{e}^{2 i T}$ with Laurent polynomials in $T$ as their coefficients.

The MGFs (2.26) in the $\eta_{j^{-}}$and $\alpha^{\prime}$-expansion of (2.16) admit similar expansions around the cusp, where the leading term is a Laurent polynomial in $y=\pi \operatorname{Im} \tau$ instead of $T$. The coefficients in the Laurent polynomials of MGFs were shown to be $\mathbb{Q}$-linear combinations of MZVs $^{15}$ [15] and are conjectured to be single-valued MZVs [10, 12]. Simple examples of the asymptotics of MGFs include

$$
\begin{aligned}
E_{2}(\tau) & =\frac{y^{2}}{45}+\frac{\zeta_{3}}{y}+\mathcal{O}\left(\mathrm{e}^{-2 y}\right) \\
\frac{\pi \bar{\nabla} E_{2}(\tau)}{y^{2}} & =\frac{2 y}{45}-\frac{\zeta_{3}}{y^{2}}+\mathcal{O}\left(\mathrm{e}^{-2 y}\right) \\
E_{3}(\tau) & =\frac{2 y^{3}}{945}+\frac{3 \zeta_{5}}{4 y^{2}}+\mathcal{O}\left(\mathrm{e}^{-2 y}\right),
\end{aligned}
$$

see (2.28) for the lattice-sum representations of the non-holomorphic Eisenstein series.

In a variety of examples, the Laurent polynomials of MGFs and B-cycle eMZVs have been related by an extension of the single-valued map $(2.7)$ to $[16,51,52]$

$$
\operatorname{sv} T=2 i y \Leftrightarrow \operatorname{sv} \log (q)=\log |q|^{2} .
$$

By (3.15) and (3.16), for instance, the Laurent polynomials of $\omega\left(0,0,2 \mid-\frac{1}{\tau}\right) \rightarrow E_{2}(\tau)$ as well as $\omega\left(0,0,1,0 \mid-\frac{1}{\tau}\right) \rightarrow-\frac{3}{8} \frac{\pi \bar{\nabla} E_{2}(\tau)}{y^{2}}$ and $\omega\left(0,0,3,0 \mid-\frac{1}{\tau}\right) \rightarrow 3 E_{3}(\tau)$ are related by (3.17).

The A-cycle eMZVs in $Z_{\vec{\eta}}^{\tau}$, by contrast, enjoy a Fourier expansion in $q=\mathrm{e}^{2 \pi i \tau}$ whose coefficients are $\mathbb{Q}\left[(2 \pi i)^{-1}\right]$ combinations of MZVs $[3,17]$ and do not feature any analogues of the Laurent polynomials in the expansion of $B_{\vec{\eta}}^{\tau}$. This is yet another indication besides their differential equations that the B-cycle integrals (3.1) are a more suitable starting point for comparison with closed-string integrals than their A-cycle counterparts (2.9).

\subsection{Single-valued correspondence of the Laurent polynomials}

As a particular convenience of the elliptic combinations (3.8) in the integrands of $\boldsymbol{J}_{\vec{\eta}}^{\tau}(\gamma \mid \rho)$, their degeneration at the cusp gives rise to Parke-Taylor factors in $n+2$ punctures $\left(\sigma_{1}=1\right.$ by $\left.z_{1}=0\right)$

$$
\sigma_{j}=\mathrm{e}^{2 \pi i z_{j}}, \quad \sigma_{+}=0, \quad \sigma_{-} \rightarrow \infty
$$

\footnotetext{
${ }^{15}$ See [12] for an earlier proof of the weaker statement that the Laurent polynomials of modular graph functions are
} $\mathbb{Q}$-linear combinations of cyclotomic MZVs. 
Since the non-holomorphic exponentials of $\Omega(z, \xi, \tau)=\exp \left(2 \pi i \xi \frac{\operatorname{Im} z}{\operatorname{Im} \tau}\right) F(z, \xi, \tau)$ cancel from the cyclic products in (3.6), one can determine the asymptotics of $V(\ldots \mid \tau)$ as $\tau \rightarrow i \infty$ by using the degeneration of the holomorphic Kronecker-Eisenstein series

$$
F\left(z_{i j}, \xi, \tau\right)=\pi \cot (\pi \xi)+i \pi \frac{\sigma_{i}+\sigma_{j}}{\sigma_{i}-\sigma_{j}}+\mathcal{O}(q)
$$

The relative factors of the $V_{w}$ in (3.8) have been engineered to obtain the following cyclic combinations of Parke-Taylor factors at the cusp,

$$
\begin{aligned}
\lim _{\tau \rightarrow i \infty} \frac{V(1,2, \ldots, n \mid \tau)}{\sigma_{1} \sigma_{2} \ldots \sigma_{n}}= & (-1)^{n-1} \lim _{\sigma_{-} \rightarrow \infty}\left|\sigma_{-}\right|^{2}[\mathrm{PT}(+, n, n-1, \ldots, 2,1,-) \\
& +\operatorname{cyc}(1,2, \ldots, n)]
\end{aligned}
$$

which have featured in the context of one-loop gauge-theory amplitudes in ambitwistor string theories [94]. The denominators on the left-hand side of (3.20) arise from $\mathrm{d} z_{j}=\frac{\mathrm{d} \sigma_{j}}{2 \pi i \sigma_{j}}$, and the factor of $\left|\sigma_{-}\right|^{2}$ on the right-hand side identifies functions on a degenerate torus with $\mathrm{SL}_{2}$-fixed expressions at genus zero [65]. Given that Parke-Taylor factors are Betti-deRham dual to disk orderings $\mathfrak{D}(\ldots)$ in (2.2), the $\tau \rightarrow i \infty$ asymptotics of $J_{\vec{\eta}}^{\tau}(\gamma \mid \rho)$ should yield the single-valued map of suitably chosen disk integrals. In fact, upon rewriting the B-cycle ordering in terms of the $\sigma_{j}$ variables (3.18), each of the contributions $\mathfrak{B}_{j}(\gamma)$ in (3.2) and figure 3 degenerates to a single disk ordering

$$
\left.\mathfrak{B}_{j}(\gamma)\right|_{\tau \rightarrow i \infty}=(-1)^{n-1} \mathfrak{D}(+, \gamma(j, j-1, \ldots, 3,2), 1, \gamma(n, n-1, \ldots, j+1),-),
$$

such that the overall B-cycle ordering $\mathfrak{B}(\gamma)=\bigoplus_{j=1}^{n} \mathfrak{B}_{j}(\gamma)$ at the cusp becomes the Betti-deRham dual to the cyclic combination of Parke-Taylor factors in (3.20),

$$
\left.\mathfrak{B}(2,3, \ldots, n)\right|_{\tau \rightarrow i \infty}=(-1)^{n-1} \bigoplus_{j=1}^{n} \mathfrak{D}(+, j, j-1, \ldots, 3,2,1, n, n-1, \ldots, j+1,-)
$$

Hence, the tree-level result (2.8) provides evidence for our central conjecture

$$
\left.\boldsymbol{J}_{\vec{\eta}}^{\tau}(\gamma \mid \rho)\right|_{\mathrm{LP}}=\left.\mathrm{sv} B_{\vec{\eta}}^{\tau}(\gamma \mid \rho)\right|_{\mathrm{LP}}
$$

where the notation $\left.\right|_{\text {LP }}$ instructs to only keep the Laurent polynomials in $\tau$ and $\operatorname{Im} \tau$ while discarding any contribution $\sim q, \bar{q}$. The conjectural part of (3.23) concerns the non-constant terms in the Laurent polynomials, i.e. corrections $\sim(\log q)^{ \pm 1}$ to the expansion around the cusp $q=0$, so it is not implied by the Betti-deRham duality of (3.2) and (3.20) which only holds at the cusp. That is why we support (3.23) by extensive tests at low orders in $\eta_{j}, \alpha^{\prime}$ as detailed below, and by the fact that the asymptotic expansions of the Green functions are related by the single-valued map with sv $\log \sigma_{i j}=\log \left|\sigma_{i j}\right|^{2}$,

$$
\begin{aligned}
\left.\mathfrak{G}_{\mathfrak{B}}\left(z_{i j}, \tau\right)\right|_{\mathrm{LP}}= & -\frac{i T}{6}-\frac{i \zeta_{2}}{T}+\frac{1}{2}\left(\log \sigma_{i}+\log \sigma_{j}\right)-\log \left|\sigma_{i j}\right| \\
& +\frac{i\left(\log \sigma_{i}-\log \sigma_{j}\right)^{2}}{4 T}
\end{aligned}
$$




$$
\begin{aligned}
\left.\mathfrak{G}_{\mathfrak{T}}\left(z_{i j}, \tau\right)\right|_{\mathrm{LP}} & =\frac{y}{3}+\log \left|\sigma_{i}\right|+\log \left|\sigma_{j}\right|-2 \log \left|\sigma_{i j}\right|+\frac{\left(\log \left|\sigma_{i}\right|-\log \left|\sigma_{j}\right|\right)^{2}}{2 y} \\
& =\left.\operatorname{sv}_{\mathfrak{B}}\left(z_{i j}, \tau\right)\right|_{\mathrm{LP}}
\end{aligned}
$$

Note that the absolute value in (3.24) is due to the argument $|u| \tau$ of $\theta_{1}$ in (3.4). For the twopoint instances $B_{\vec{\eta}}^{\tau}$ and $J_{\vec{\eta}}^{\tau}$ of the open- and closed-string integrals (3.1) and (3.13), the Laurent polynomials in the asymptotics at the cusp can be determined [95] by mild generalizations of the techniques in $[16,55]$ (also see [56] for an alternative approach to the closed-string case):

$$
\begin{aligned}
\left.B_{\eta}^{\tau}(2 \mid 2)\right|_{\mathrm{LP}}= & \exp \left(-\frac{i s_{12} T}{6}-\frac{i s_{12} \zeta_{2}}{T}\right)\left\{\left[i \cot \left(\eta_{2} T\right)+1\right] \exp \left(\frac{i s_{12}}{4 T} \partial_{\eta_{2}}^{2}\right) \frac{1}{s_{12}+2 \eta_{2}}\right. \\
& \times\left[\frac{\Gamma\left(1+\frac{s_{12}}{2}+\eta_{2}\right) \Gamma\left(1-s_{12}\right)}{\Gamma\left(1-\frac{s_{12}}{2}+\eta_{2}\right)}-\mathrm{e}^{i T\left(\frac{s_{12}}{2}+\eta_{2}\right)}\right]+\left[i \cot \left(\eta_{2} T\right)-1\right] \\
& \times \exp \left(\frac{i s_{12}}{4 T} \partial_{\eta_{2}}^{2}\right) \frac{1}{s_{12}-2 \eta_{2}}\left[\frac{\Gamma\left(1+\frac{s_{12}}{2}-\eta_{2}\right) \Gamma\left(1-s_{12}\right)}{\Gamma\left(1-\frac{s_{12}}{2}-\eta_{2}\right)}-\mathrm{e}^{i T\left(\frac{s_{12}}{2}-\eta_{2}\right)}\right] \\
& +\frac{1}{s_{12}} \exp \left(\frac{i s_{12}}{4 T} \partial_{\eta_{2}}^{2}\right)\left[\frac{\Gamma\left(1+\frac{s_{12}}{2}-\eta_{2}\right) \Gamma\left(1-s_{12}\right)}{\Gamma\left(1-\frac{s_{12}}{2}-\eta_{2}\right)}\right. \\
& \left.\left.-\frac{\Gamma\left(1+\frac{s_{12}}{2}+\eta_{2}\right) \Gamma\left(1-s_{12}\right)}{\Gamma\left(1-\frac{s_{12}}{2}+\eta_{2}\right)}\right]\right\} \\
\left.J_{\eta}^{\tau}(2 \mid 2)\right|_{\mathrm{LP}}= & \exp \left(\frac{s_{12} y}{3}\right)\left\{\left[i \cot \left(2 i \eta_{2} y\right)+1\right] \exp \left(\frac{s_{12}}{8 y} \partial_{\eta_{2}}^{2}\right) \frac{1}{s_{12}+2 \eta_{2}}\right. \\
& \times\left[\frac{\Gamma\left(1+\frac{s_{12}}{2}+\eta_{2}\right) \Gamma\left(1-s_{12}\right) \Gamma\left(1+\frac{s_{12}}{2}-\eta_{2}\right)}{\Gamma\left(1-\frac{s_{12}}{2}+\eta_{2}\right) \Gamma\left(1+s_{12}\right) \Gamma\left(1-\frac{s_{12}}{2}-\eta_{2}\right)}-\mathrm{e}^{-y\left(s_{12}+2 \eta_{2}\right)}\right] \\
& +\left[i \cot \left(2 i \eta_{2} y\right)-1\right] \exp \left(\frac{s_{12}}{8 y} \partial_{\eta_{2}}^{2}\right) \frac{1}{s_{12}-2 \eta_{2}} \\
& \left.\times\left[\frac{\Gamma\left(1+\frac{s_{12}}{2}+\eta_{2}\right) \Gamma\left(1-s_{12}\right) \Gamma\left(1+\frac{s_{12}}{2}-\eta_{2}\right)}{\Gamma\left(1-\frac{s_{12}}{2}+\eta_{2}\right) \Gamma\left(1+s_{12}\right) \Gamma\left(1-\frac{s_{12}}{2}-\eta_{2}\right)}-\mathrm{e}^{-y\left(s_{12}-2 \eta_{2}\right)}\right]\right\}
\end{aligned}
$$

These two-point expressions are easily seen to line up with the all-multiplicity claim (3.23) since

$$
\operatorname{sv}\left[\frac{\Gamma(1-a) \Gamma(1-b)}{\Gamma(1-a-b)}\right]=\frac{\Gamma(1-a) \Gamma(1-b) \Gamma(1+a+b)}{\Gamma(1+a) \Gamma(1+b) \Gamma(1-a-b)},
$$

and the last line of (3.26) therefore vanishes under sv. Moreover, we have checked the threepoint Laurent polynomials to obey (3.23) to the orders in the $s_{i j^{-}}$and $\eta_{j}$-expansions where MGFs such as (2.26) of total modular weight 10 occur $^{16}$. Finally, we have checked (3.23) to hold at four points to the orders where MGFs of total modular weight 8 occur, at least for

\footnotetext{
${ }^{16}$ This amounts to performing the $\alpha^{\prime}$ - and $\eta$-expansion to order 10 in the terminology of section 3.4.2 of [18].
} 
contributions from $\varphi_{\vec{\eta}}^{\tau}$ in (2.13) without any singular factors of $f^{(1)}\left(z_{i j}, \tau\right) .{ }^{17}$ These checks are based on Enriquez' methods [3] (also see appendix B of [51]) to determine the Laurent polynomials of B-cycle eMZVs. The Laurent polynomials for all B-cycle eMZVs with (length + weight $) \leqslant 16$ obtained from an FORM implementation [96] of these methods are available for download [97].

While the two-point Laurent-polynomials generated by (3.26) and (3.27) only involve Riemann zeta values, higher-point examples also introduce irreducible MZVs of depth $\geqslant 2$. The appearance of $\zeta_{3,5}$ in B-cycle Laurent polynomials is later on exemplified in (4.27) and (5.37). Moreover, the appearance of $\zeta_{3,5,3}$ in open- and closed-string calculations at three points in agreement with (3.23) was observed in section 3.3.5 of [51], based on earlier closed-string computations [12].

\subsection{Single-valued correspondence of the differential equations}

The holomorphic derivatives of the $B_{\vec{\eta}}^{\tau}$ and $J_{\vec{\eta}}^{\tau}$-integrals (3.1) and (3.13) can be easily deduced from (2.19): in the open-string case, the modular $S$ transformation relating $B_{\vec{\eta}}^{\tau}=Z_{\vec{\eta}}^{-1 / \tau}$ and the modular weight $(k, 0)$ of the holomorphic Eisenstein series $G_{k}$ give rise to

$$
\frac{\partial}{\partial \log q} B_{\vec{\eta}}^{\tau}(\gamma \mid \rho)=\frac{1}{(2 \pi i)^{2}} \sum_{k=0}^{\infty}(1-k) \tau^{k-2} G_{k}(\tau) \sum_{\alpha \in S_{n-1}} r_{\vec{\eta}}\left(\epsilon_{k}\right)_{\rho}^{\alpha} B_{\vec{\eta}}^{\tau}(\gamma \mid \alpha),
$$

see [60] for the $n$-point derivations $r_{\vec{\eta}}\left(\epsilon_{k}\right)$ and (2.21) for their two-point examples. Since the single-valued map at genus zero acts on transcendental constants, we have passed to the differential operator $\frac{\partial}{\partial \log q}=(2 \pi i)^{-1} \partial_{\tau}$ in comparison to (2.19) and in preparation for the extended single-valued map to be introduced below around (3.32).

In the closed-string case, the $\overline{V(\ldots \mid \tau)}$ in (3.13) are not affected by holomorphic derivatives, and one can import a simplified version of the differential equations in [61] where contributions $\sim \bar{\eta}_{j} \partial_{\eta_{j}}$ are absent,

$$
\begin{aligned}
\frac{\partial}{\partial \log q} J_{\vec{\eta}}^{\tau}(\gamma \mid \rho)= & \frac{1}{(2 \pi i)^{2}} \sum_{k=0}^{\infty}(1-k)(\tau-\bar{\tau})^{k-2} G_{k}(\tau) \\
& \times \sum_{\alpha \in S_{n-1}} \operatorname{sv} r_{\vec{\eta}}\left(\epsilon_{k}\right)_{\rho}{ }^{\alpha} J_{\vec{\eta}}^{\tau}(\gamma \mid \alpha) .
\end{aligned}
$$

By the differential equation (2.18) of the Green functions, also the term $\sim \zeta_{2}$ in $r_{\vec{\eta}}\left(\epsilon_{0}\right)$ is absent which we have indicated through the sv notation,

$$
\operatorname{sv} r_{\vec{\eta}}\left(\epsilon_{k}\right)= \begin{cases}r_{\vec{\eta}}\left(\epsilon_{k}\right) & : k \geqslant 4 \\ r_{\vec{\eta}}\left(\epsilon_{0}\right)-2 \zeta_{2} s_{12 \ldots n} & : k=0\end{cases}
$$

where $r_{\vec{\eta}}\left(\epsilon_{2}\right)=0$. The building blocks of the closed-string differential operator in (3.30) are related to those in the open-string analogue (3.29) through an extension SV of the single-valued

\footnotetext{
${ }^{17}$ We have excluded the singular functions $f^{(1)}\left(z_{i j}, \tau\right)=\frac{1}{z_{i j}}+\mathcal{O}\left(z_{i j}\right)$ in the integrand from our checks to avoid the tedious treatment of the resulting kinematic poles in the $\alpha^{\prime}$-expansion. For the contributions of $V_{0}$ and $V_{1}$ to the integrand $\overline{V(1,2,3,4 \mid \tau)}$ in (3.9), we have checked the Laurent polynomials from up to one factor of $f^{(1)}\left(z_{i j}, \tau\right)$ in the

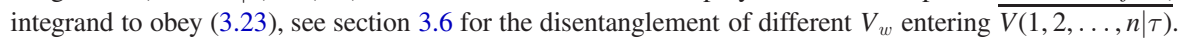


map (recall that $T=\pi \tau$ and $y=\pi \operatorname{Im} \tau$ )

$$
\begin{aligned}
\mathrm{SV}\left[(2 \pi i \tau)^{k-2} \frac{G_{k}(\tau)}{(2 \pi i)^{k}} r_{\vec{\eta}}\left(\epsilon_{k}\right)\right] & =\mathrm{SV}\left[(2 i T)^{k-2}\right] \mathrm{SV}\left[\frac{G_{k}(\tau)}{(2 \pi i)^{k}}\right] \mathrm{SV}\left[r_{\vec{\eta}}\left(\epsilon_{k}\right)\right] \\
& =(-4 y)^{k-2} \frac{G_{k}(\tau)}{(2 \pi i)^{k}} \operatorname{sV} r_{\vec{\eta}}\left(\epsilon_{k}\right) \\
& =\frac{1}{(2 \pi i)^{2}}(\tau-\bar{\tau})^{k-2} G_{k}(\tau) \operatorname{sV} r_{\vec{\eta}}\left(\epsilon_{k}\right)
\end{aligned}
$$

which is taken to preserve the properties of sv,

$$
\mathrm{SV} \zeta_{n_{1}, n_{2}, \ldots, n_{r}}=\operatorname{sv} \zeta_{n_{1}, n_{2}, \ldots, n_{r}}, \quad \mathrm{SV} T=2 i y
$$

and to furthermore preserve $(2 \pi i)^{-k} G_{k}(\tau)$ (the inverse powers of $\pi$ ensuring rational coefficients in the $q$-expansion) and the $\eta_{j}$-variables, cf (3.32). In other words, the differential operator $\mathcal{O}_{\vec{\eta}}^{\tau}=(2 \pi i)^{-2} \sum_{k=0}^{\infty}(1-k) \tau^{k-2} G_{k}(\tau) r_{\vec{\eta}}\left(\epsilon_{k}\right)$ appearing in (3.29) and its closed-string analogue in (3.30) are related by

$$
\begin{aligned}
& \frac{\partial B_{\vec{\eta}}^{\tau}(\gamma \mid \rho)}{\partial \log q}=\sum_{\alpha \in S_{n-1}} \mathcal{O}_{\vec{\eta}}^{\tau}(\rho \mid \alpha) B_{\vec{\eta}}^{\tau}(\gamma \mid \alpha) \leftrightarrow \\
& \frac{\partial J_{\vec{\eta}}^{\tau}(\gamma \mid \rho)}{\partial \log q}=\sum_{\alpha \in S_{n-1}}\left[\operatorname{SV~}_{\vec{\eta}}^{\tau}(\rho \mid \alpha)\right] \boldsymbol{J}_{\vec{\eta}}^{\tau}(\gamma \mid \alpha) .
\end{aligned}
$$

From the above discussion, both the $\tau \rightarrow i \infty$ asymptotics and the differential operators of the open- and closed-string integrals $B_{\vec{\eta}}^{\tau}$ and $J_{\vec{\eta}}^{\tau}$ are related by the SV map (3.33). Hence, we propose that the solutions of (3.34) yield an appropriate extension of the SV map

$$
J_{\vec{\eta}}^{\tau}(\gamma \mid \rho)=\operatorname{SVB} B_{\vec{\eta}}^{\tau}(\gamma \mid \rho)
$$

This proposal is key the result of this work, relating the open-string integrals $B_{\vec{\eta}}^{\tau}(\gamma \mid \rho)$ in (3.1) with integration ordering $\gamma$ to the closed-string integrals $J_{\vec{\eta}}^{\tau}(\gamma \mid \rho)$, where the ordering $\gamma$ governs the singularity structure of the antielliptic integrand $\bar{V}$ in (3.13). By construction, this $\mathrm{SV}$ map commutes with the holomorphic $\tau$-derivative and, under the assumption (3.23), it is consistent at the level of the Laurent polynomials at the cusp. Compatibility of a single-valued map at genus one with $\tau \rightarrow i \infty$ generalizes the fact that the single-valued map of multiple polylogarithms commutes with evaluation [46]. Moreover, by the evidence to be discussed in section 4.3, the SV map is expected to be compatible with the shuffle product. As we will see in the next section, the $\alpha^{\prime}$-expansion of (3.35) induces an elliptic single-valued map for the eMZVs generated by $B_{\vec{\eta}}^{\tau}$ which yields the MGFs generated by $\boldsymbol{J}_{\vec{\eta}}^{\tau}$.

Let us consider the scope of our definition (3.35). Firstly, not all the holomorphic iterated Eisenstein integrals appear in the $\alpha^{\prime}$-expansion of $B_{\vec{\eta}}^{\tau}$. As was discussed in $[17,18]$ and will become clearer when we discuss the $\alpha^{\prime}$-expansion of the solution of (3.34), relations among the $r_{\vec{\eta}}\left(\epsilon_{k}\right)$ such as (2.22) lead to dropouts of certain iterated Eisenstein integrals from eMZVs and $Y_{\vec{\eta}}^{\tau}$ and thereby from $B_{\vec{\eta}}^{\tau}$ and $J_{\vec{\eta}}^{\tau}$. Hence, (3.35) does not comprise the SV map for the combinations of iterated Eisenstein integrals affected by these dropouts, starting with double integrals involving $G_{4}$ and $G_{10}$.

By contrast, the SV map of arbitrary convergent eMZVs can be extracted from (3.35) at sufficiently high multiplicity: as will be detailed in section 5.7, for $\omega\left(n_{1}, \ldots, n_{r} \mid \tau\right)$ in (2.24) with 
given entries $n_{j}$ (where $n_{1}, n_{r} \neq 1$ ), one can engineer a combination of genus-one open-string integrals, where the desired eMZV occurs at the zeroth order in $s_{i j}{ }^{18}$

Finally, one could wonder whether holomorphic cusp forms lead to ambiguities in the definition of the $V(1, \ldots, n \mid \tau)$ in (3.8): ${ }^{19}$ Starting from $n=14$ points, their defining properties including simple-pole residues, the modularity of their constituents and their behavior (3.20) at the cusp are unchanged when adding combinations of holomorphic cusp forms and lower-weight $V_{w}(1, \ldots, n \mid \tau)$. However, adding a cusp form without any $z_{j}$-dependent coefficient to $V(1, \ldots, n \mid \tau)$ leads to a contradiction with the requirement that the $\tau$-independent $\eta^{1-n}$ order of $B_{\vec{\eta}}^{\tau}$ is mapped to the same term $\sim \eta^{1-n}$ in the corresponding $J_{\vec{\eta}}^{\tau}$ integral. Products of $V_{w}(1, \ldots, n \mid \tau)$ with cusp forms in turn would violate the pole structure (3.12) that reflects the boundary structure of the dual cycles. Hence, the above requirements do not leave any room to modify $V(1, \ldots, n \mid \tau)$ by holomorphic cusp forms.

\subsection{Dual modular weights for cycles}

Given that the antielliptic $\overline{V_{w}(\ldots \mid \tau)}$-functions (3.6) carry modular weight $(0, w)$, their combinations $\overline{V(\ldots \mid \tau)}(3.8)$ mix different modular weights. Hence, the $\alpha^{\prime}$-expansion of the generating function (3.13) with $\overline{V(\ldots \mid \tau)}$ in the integrand mixes modular forms of different weight, even at fixed order in $\eta_{j}$. One may wish to isolate the contributions at fixed modular weights and study

$$
\begin{aligned}
J_{w, \vec{\eta}}^{\tau}(\gamma \mid \rho)= & \frac{(2 i)^{n-1}}{(-2 \pi i)^{w}} \int_{\mathfrak{T}^{n-1}}\left(\prod_{j=2}^{n} \mathrm{~d}^{2} z_{j}\right) \prod_{1 \leqslant i<j}^{n} \mathrm{e}^{s_{i j} \mathcal{G}_{\mathfrak{T}}\left(z_{i j}, \tau\right)} \\
& \times \overline{V_{w}(1, \gamma(2, \ldots, n) \mid \tau)} \varphi_{(\tau-\bar{\tau}) \vec{\eta}}^{\tau}(1, \rho(2, \ldots, n)),
\end{aligned}
$$

with $0 \leqslant w \leqslant n-2$, where the terms at homogeneity degree $m$ in the $\eta_{j}$ are modular forms of weight $(0,1-n-m+w)$. One can still identify combinations of integration cycles (3.2) to write (3.36) at fixed modular weight $w$ and ordering $\gamma$ as the single-valued version of known open-string integrals: each $V_{w}(1,2, \ldots, n \mid \tau)$ with $w \leqslant n-2$ is expressible via permutation sums

$$
V_{w}(1,2, \ldots, n \mid \tau)=(2 \pi i)^{w} \sum_{\gamma \in S_{n-1}} c_{w, \gamma} V(1, \gamma(2, \ldots, n))
$$

with coefficients $c_{w, \gamma} \in \mathbb{Q}$, e.g.

$$
\begin{aligned}
V_{0}(1, \ldots, n \mid \tau)= & 1=\sum_{\gamma \in S_{n-1}} V(1, \gamma(2, \ldots, n)) \\
V_{1}(1,2,3 \mid \tau)= & i \pi[V(1,2,3 \mid \tau)-V(1,3,2 \mid \tau)] \\
V_{1}(1,2,3,4 \mid \tau)= & 2 \pi i[V(1,2,3,4 \mid \tau)-V(1,4,3,2 \mid \tau)] \\
V_{2}(1,2,3,4 \mid \tau)= & \frac{(2 \pi i)^{2}}{6}[2 V(1,2,3,4 \mid \tau)+2 V(1,4,3,2 \mid \tau)-V(1,2,4,3 \mid \tau) \\
& -V(1,3,4,2 \mid \tau)-V(1,3,2,4 \mid \tau)-V(1,4,2,3 \mid \tau)]
\end{aligned}
$$

\footnotetext{
${ }^{18}$ By a similar argument, each MGF can be realized in the $s_{i j}^{0}$-order of $Y^{\tau}$-integrals at sufficiently high multiplicity, see section 2.5 of [61].

${ }^{19}$ We are grateful to Nils Matthes for valuable discussions on this point.
} 
Table 1. Examples of the unsigned Stirling numbers $S_{n-1, n-w-1}$ which count the number of independent permutations $\gamma \in S_{n-1}$ of $V_{w}(1, \gamma(2, \ldots, n) \mid \tau)$.

\begin{tabular}{ccccccc}
\hline & \multicolumn{7}{c}{$w$} \\
\cline { 2 - 7 }$n$ & 0 & 1 & 2 & 3 & 4 & 5 \\
\hline 2 & 1 & 0 & 0 & 0 & 0 & 0 \\
3 & 1 & 1 & 0 & 0 & 0 & 0 \\
4 & 1 & 3 & 2 & 0 & 0 & 0 \\
5 & 1 & 6 & 11 & 6 & 0 & 0 \\
6 & 1 & 10 & 35 & 50 & 24 & 0 \\
7 & 1 & 15 & 85 & 225 & 274 & 120 \\
\hline
\end{tabular}

These relations and coefficients $c_{w, \gamma}$ can be traced back to the symmetries of the $V_{w}$-functions including the cyclicity (3.14), the reflection property

$$
V_{w}(1,2, \ldots, n \mid \tau)=(-1)^{w} V_{w}(n, \ldots, 2,1 \mid \tau)
$$

and corollaries of the Fay identity [98] which have been discussed in [79, 99]. An independent method based on the degeneration (3.20) to determine the $c_{w, \gamma}$ is described in appendix A. As a result, there are less than $(n-1)$ ! independent permutations $V_{w}(1, \gamma(2, \ldots, n) \mid \tau)$ at fixed $0 \leqslant w \leqslant n-2$ and $n \geqslant 3$. Their counting is governed by the unsigned Stirling number $S_{n-1, n-w-1}$ of the first kind (where $S_{a, b}$ counts the number of permutations of $a$ elements with $b$ disjoint cycles) as exemplified in table 1 .

In particular, permutations of $V_{w=n-2}(1, \ldots, n \mid \tau)$ are related by Kleiss-Kuijf relations $[99,100]$

$$
V_{n-2}\left(1,\left(a_{2}, \ldots, a_{j}\right) \sqcup\left(a_{j+1}, \ldots, a_{n}\right) \mid \tau\right)=0, \quad j=2,3, \ldots, n-1
$$

such as

$$
V_{1}(1,2,3 \mid \tau)=-V_{1}(1,3,2 \mid \tau), \quad V_{2}(1,2,3,4 \mid \tau)+\operatorname{cyc}(2,3,4)=0,
$$

consistent with the counting $S_{n-1,1}=(n-2)$ ! of independent permutations.

Given the decomposition (3.37) of a given $V_{w}$ function with rational coefficients $c_{w, \gamma}$, one can by (3.35) write each $J_{w, \vec{\eta}}^{\tau}$ integral (3.36) as a combination of single-valued B-cycle integrals

$$
\boldsymbol{J}_{w, \vec{\eta}}^{\tau}(2, \ldots, n \mid \rho)=\mathrm{SV} \sum_{\gamma \in S_{n-1}} c_{w, \gamma} \boldsymbol{B}_{\vec{\eta}}^{\tau}(\gamma \mid \rho) .
$$

For instance, the equivalent

$$
\begin{aligned}
& J_{0, \eta_{2}, \eta_{3}}^{\tau}(2,3 \mid \rho)=\operatorname{SV}\left[B_{\eta_{2}, \eta_{3}}^{\tau}(2,3 \mid \rho)+B_{\eta_{2}, \eta_{3}}^{\tau}(3,2 \mid \rho)\right] \\
& J_{1, \eta_{2}, \eta_{3}}^{\tau}(2,3 \mid \rho)=\frac{1}{2} \operatorname{SV}\left[B_{\eta_{2}, \eta_{3}}^{\tau}(2,3 \mid \rho)-B_{\eta_{2}, \eta_{3}}^{\tau}(3,2 \mid \rho)\right]
\end{aligned}
$$

of (3.35) together with (3.38) and (3.39) suggests to assign a formal 'dual modular weight' 0 and 1 to the symmetric and antisymmetric three-point cycles, respectively,

$$
\begin{aligned}
\mathfrak{B}(2,3)+\mathfrak{B}(3,2) \leftrightarrow \text { dual modular weight } 0 \\
\mathfrak{B}(2,3)-\mathfrak{B}(3,2) \leftrightarrow \text { dual modular weight } 1 .
\end{aligned}
$$


Similarly, combining (3.35) with (3.38), (3.40) and (3.41) leads to the following dual modular weights (d.m.w.) for four-point cycles

$$
\begin{array}{r}
\mathfrak{B}(2,3,4)+\mathfrak{B}(4,3,2)+\mathfrak{B}(2,4,3)+\mathfrak{B}(3,4,2)+\mathfrak{B}(3,2,4)+\mathfrak{B}(4,2,3) \leftrightarrow \text { d.m.w. } 0 \\
\mathfrak{B}(2,3,4)-\mathfrak{B}(4,3,2) \leftrightarrow \text { d.m.w. } 1 \\
2 \mathfrak{B}(2,3,4)+2 \mathfrak{B}(4,3,2)-\mathfrak{B}(2,4,3)-\mathfrak{B}(3,4,2)-\mathfrak{B}(3,2,4)-\mathfrak{B}(4,2,3) \leftrightarrow \text { d.m.w. } 2,
\end{array}
$$

see section 5.5 for a more detailed discussion of the weight-two case. Finally, the allmultiplicity formula (3.37) translates into

$$
\sum_{\gamma \in S_{n-1}} c_{w, \gamma} \mathfrak{B}(\gamma(2,3, \ldots, n)) \leftrightarrow \text { dual modular weight } w
$$

see appendix A for the rational coefficients $c_{w, \gamma}$ and table 1 for the counting of independent $n$-point cycles with dual modular weight $w$.

\section{Single-valued iterated Eisenstein integrals from $\alpha^{\prime}$-expansions}

The goal of this section is to provide the explicit form of the single-valued map SV for the iterated-Eisenstein-integral representation of eMZVs [17] by reading (3.35) at the level of the $\alpha^{\prime}$ - and $\eta_{j}$-expansions of $B_{\vec{\eta}}^{\tau}$ and $J_{\vec{\eta}}^{\tau}$. We will employ the formulation of iterated Eisenstein integrals with integration kernels $\tau^{j} G_{k}, k \geqslant 4$ [101],

$$
\mathcal{E}\left[\begin{array}{l}
j_{1} j_{2} \ldots j_{\ell} \\
k_{1} k_{2} \ldots k_{\ell}
\end{array} ;\right]=(-1)^{\ell} \int_{0<q_{1}<q_{2}<\cdots<q_{\ell}<q} \frac{\mathrm{d} q_{1}}{q_{1}} \frac{\mathrm{d} q_{2}}{q_{2}} \cdots \frac{\mathrm{d} q_{\ell}}{q_{\ell}} \prod_{r=1}^{\ell} \frac{\left(2 \pi i \tau_{r}\right)^{j_{r}} G_{k_{r}}\left(\tau_{r}\right)}{(2 \pi i)^{k_{r}}} .
$$

The entries are taken to obey $k_{r} \geqslant 4$ and $0 \leqslant j_{r} \leqslant k_{r}-2$, and we use tangential-base-point regularization for the divergences as $q_{r} \rightarrow 0$ [101], which implies that the iterated Eisenstein integrals $\mathcal{E}[\ldots ; \tau]$ vanish in the regularized limit $\tau \rightarrow i \infty$.

\subsection{Improving the differential equations}

We shall now derive the structure of the $\alpha^{\prime}$-expansion of $B_{\vec{\eta}}^{\tau}$ and $J_{\vec{\eta}}^{\tau}$ by repeating the key steps of [18] in solving the differential equation (2.19) of $Y_{\vec{\eta}}^{\tau}$. The first step is to introduce redefined generating series $\widehat{B}_{\vec{\eta}}^{\tau}$ and $\widehat{J}_{\vec{\eta}}^{\tau}$ by

$$
\widehat{B}_{\vec{\eta}}^{\tau}=\exp \left(-\frac{r_{\vec{\eta}}\left(\epsilon_{0}\right)}{2 \pi i \tau}\right) B_{\vec{\eta}}^{\tau}, \quad \widehat{J}_{\vec{\eta}}^{\tau}=\exp \left(-\frac{\operatorname{sv} r_{\vec{\eta}}\left(\epsilon_{0}\right)}{2 \pi i(\tau-\bar{\tau})}\right) J_{\vec{\eta}}^{\tau}
$$

After this redefinition, the $k=0$ terms involving $\frac{G_{0}}{\tau^{2}}$ and $\frac{G_{0}}{(\tau-\bar{\tau})^{2}}$ with $G_{0}=-1$ are absent from the analogues of the differential equations (3.29) and (3.30), see (4.4) and (4.5) below. Throughout this section, we suppress the permutations $\gamma, \rho$ labeling $\boldsymbol{B}_{\vec{\eta}}^{\tau}(\gamma \mid \rho)$ and $\boldsymbol{J}_{\vec{\eta}}^{\tau}(\gamma \mid \rho)$, and all matrix representations $r_{\vec{\eta}}\left(\epsilon_{k}\right)$ are understood to act matrix-multiplicatively on the $\rho$-entry.

Since the $r_{\vec{\eta}}\left(\epsilon_{k}\right)$ are expected (as tested for a wide range of $k$ and $n$ ) to inherit the ad-nilpotency relations of the derivation algebra,

$$
\operatorname{ad}_{r_{\vec{\eta}}\left(\epsilon_{0}\right)}^{k-1} r_{\vec{\eta}}\left(\epsilon_{k}\right)=0, \quad k \geqslant 4
$$


the combinations $\exp \left(-\frac{r_{\vec{\eta}}\left(\epsilon_{0}\right)}{2 \pi i \tau}\right) r_{\vec{\eta}}\left(\epsilon_{k \geqslant 4}\right) \exp \left(\frac{r_{\vec{\eta}}\left(\epsilon_{0}\right)}{2 \pi i \tau}\right)$ in the differential equations of the redefined integrals (4.2) truncate to a finite number of terms and we obtain

$$
\begin{aligned}
& 2 \pi i \partial_{\tau} \widehat{\boldsymbol{B}}_{\vec{\eta}}^{\tau}=\sum_{k=4}^{\infty}(1-k) \sum_{j=0}^{k-2} \frac{1}{j !}\left(\frac{-1}{2 \pi i}\right)^{j} \tau^{k-2-j} G_{k}(\tau) r_{\vec{\eta}}\left(\operatorname{ad}_{\epsilon_{0}}^{j}\left(\epsilon_{k}\right)\right) \widehat{B}_{\vec{\eta}}^{\tau} \\
& 2 \pi i \partial_{\tau} \widehat{J}_{\vec{\eta}}^{\tau}=\sum_{k=4}^{\infty}(1-k) \sum_{j=0}^{k-2} \frac{1}{j !}\left(\frac{-1}{2 \pi i}\right)^{j}(\tau-\bar{\tau})^{k-2-j} G_{k}(\tau) r_{\vec{\eta}}\left(\operatorname{ad}_{\epsilon_{0}}^{j}\left(\epsilon_{k}\right)\right) \widehat{\boldsymbol{J}}_{\vec{\eta}}^{\tau} .
\end{aligned}
$$

We have used that $\operatorname{ad}_{r_{\vec{\eta}}\left(\epsilon_{0}\right)}(\cdot)=\left[r_{\vec{\eta}}\left(\epsilon_{0}\right), \cdot\right]=\operatorname{ad}_{\mathrm{sv} r_{\vec{\eta}}\left(\epsilon_{0}\right)}(\cdot)\left(\right.$ since the term $\sim \zeta_{2}$ in (3.31) suppressed by sv is commutative) and employ the shorthands

$$
r_{\vec{\eta}}\left(\operatorname{ad}_{\epsilon_{0}}^{j}\left(\epsilon_{k}\right)\right)=\operatorname{ad}_{r_{\vec{\eta}}\left(\epsilon_{0}\right)}^{j} r_{\vec{\eta}}\left(\epsilon_{k}\right), \quad r_{\vec{\eta}}\left(\epsilon_{k_{1}} \epsilon_{k_{2}}\right)=r_{\vec{\eta}}\left(\epsilon_{k_{1}}\right) r_{\vec{\eta}}\left(\epsilon_{k_{2}}\right)
$$

In equations (4.4), (4.5) and below, we use the derivative with respect to $\tau$ instead of $\log q$ as compared to section 3.5 above.

\subsection{The $\alpha^{\prime}$-expansion of $B_{\vec{\eta}}^{\tau}$}

By the differential equation

$$
2 \pi i \partial_{\tau} \mathcal{E}\left[\begin{array}{llll}
j_{1} & j_{2} & \ldots & j_{\ell} \\
k_{1} & k_{2} & \ldots & k_{\ell}
\end{array}\right]=-(2 \pi i)^{2-k_{\ell}}(2 \pi i \tau)^{j_{\ell}} G_{k_{\ell}}(\tau) \mathcal{E}\left[\begin{array}{llll}
j_{1} & j_{2} & \ldots & j_{\ell-1} \\
k_{1} & k_{2} & \ldots & k_{\ell-1}
\end{array}\right]
$$

of the iterated Eisenstein integrals (4.1), one can solve the differential equation (4.4) of the generating series through the path-ordered exponential

$$
\begin{aligned}
\widehat{B}_{\vec{\eta}}^{\tau}= & \sum_{\ell=0}^{\infty} \sum_{\substack{k_{1}, k_{2}, \ldots, k_{\ell} \\
=4,6,8, \ldots}} \sum_{j_{1}=0}^{k_{1}-2} \sum_{j_{2}=0}^{k_{2}-2} \ldots \sum_{j_{\ell}=0}^{k_{\ell}-2}\left(\prod_{i=1}^{\ell} \frac{(-1)^{j_{i}}\left(k_{i}-1\right)}{\left(k_{i}-j_{i}-2\right) !}\right) \mathcal{E}\left[\begin{array}{llll}
j_{1} & j_{2} & \ldots & j_{\ell} \\
k_{1} & k_{2} & \ldots & k_{\ell}
\end{array}\right] \\
& \times r_{\vec{\eta}}\left(\operatorname{ad}_{\epsilon_{0}}^{k_{\ell}-j_{\ell}-2}\left(\epsilon_{k_{\ell}}\right) \ldots \operatorname{ad}_{\epsilon_{0}}^{k_{2}-j_{2}-2}\left(\epsilon_{k_{2}}\right) \operatorname{ad}_{\epsilon_{0}}^{k_{1}-j_{1}-2}\left(\epsilon_{k_{1}}\right)\right) \widehat{B}_{\vec{\eta}}^{i \infty}
\end{aligned}
$$

for some initial value $\widehat{B}_{\vec{\eta}}^{i \infty}$ to be discussed below. By inverting the redefinition (4.2) and moving the exponential to act directly on the initial value, we obtain the open-string analogue

$$
\begin{aligned}
B_{\vec{\eta}}^{\tau}= & \sum_{\ell=0}^{\infty} \sum_{\substack{k_{1}, k_{2}, \ldots, k_{\ell} \\
=4,6,8, \ldots}} \sum_{j_{1}=0}^{k_{1}-2} \sum_{j_{2}=0}^{k_{2}-2} \ldots \sum_{j_{\ell}=0}^{k_{\ell}-2}\left(\prod_{i=1}^{\ell} \frac{(-1)^{j_{i}}\left(k_{i}-1\right)}{\left(k_{i}-j_{i}-2\right) !}\right) \beta\left[\begin{array}{cccc}
j_{1} & j_{2} & \ldots & j_{\ell} \\
k_{1} & k_{2} & \ldots & k_{\ell}
\end{array}\right] \\
& \times r_{\vec{\eta}}\left(\operatorname{ad}_{\epsilon_{0}}^{k_{\ell}-j_{\ell}-2}\left(\epsilon_{k_{\ell}}\right) \ldots \operatorname{ad}_{\epsilon_{0}}^{k_{2}-j_{2}-2}\left(\epsilon_{k_{2}}\right) \operatorname{ad}_{\epsilon_{0}}^{k_{1}-j_{1}-2}\left(\epsilon_{k_{1}}\right)\right) \exp \left(\frac{r_{\vec{\eta}}\left(\epsilon_{0}\right)}{2 \pi i \tau}\right) \widehat{B}_{\vec{\eta}}^{i \infty}
\end{aligned}
$$

of the key result for the $\alpha^{\prime}$-expansion of $Y_{\vec{\eta}}^{\tau}$ in (3.11) of [18]. In commuting $\exp \left(\frac{r_{\bar{\eta}}\left(\epsilon_{0}\right)}{2 \pi i \tau}\right)$ past the $\epsilon_{k_{j}}$, the iterated Eisenstein integrals are rearranged into the combinations

$$
\beta\left[\begin{array}{l}
j_{1} \\
k_{1}
\end{array} \tau\right]=\sum_{p_{1}=0}^{k_{1}-j_{1}-2}\left(\begin{array}{c}
k_{1}-j_{1}-2 \\
p_{1}
\end{array}\right)\left(\frac{i}{2 T}\right)^{p_{1}} \mathcal{E}\left[\begin{array}{c}
j_{1}+p_{1} \\
k_{1}
\end{array} \tau\right]
$$


and more generally

$$
\begin{aligned}
\beta\left[\begin{array}{llll}
j_{1} & j_{2} & \ldots & j_{\ell} \\
k_{1} & k_{2} & \ldots & k_{\ell}
\end{array} ;\right]= & \sum_{p_{1}=0}^{k_{1}-j_{1}-2} \sum_{p_{2}=0}^{k_{2}-j_{2}-2} \ldots \sum_{p_{\ell}=0}^{k_{\ell}-j_{\ell}-2}\left(\begin{array}{c}
k_{1}-j_{1}-2 \\
p_{1}
\end{array}\right) \\
& \times\left(\begin{array}{c}
k_{2}-j_{2}-2 \\
p_{2}
\end{array}\right) \cdots\left(\begin{array}{c}
k_{\ell}-j_{\ell}-2 \\
p_{\ell}
\end{array}\right) \\
& \times\left(\frac{i}{2 T}\right)^{p_{1}+p_{2}+\cdots+p_{\ell}} \mathcal{E}\left[\begin{array}{cccc}
j_{1}+p_{1} & j_{2}+p_{2} & \ldots & j_{\ell}+p_{\ell} ; \tau \\
k_{1} & k_{2} & \ldots & k_{\ell}
\end{array}\right] .
\end{aligned}
$$

Note that (4.9) is an alternative ${ }^{20}$ organization of open-string $\alpha^{\prime}$-expansions at genus one as compared to $[59,60]$. Non-planar B-cycle integrals obey the same differential equation (3.29) as the planar ones and therefore have an $\alpha^{\prime}$-expansion of the same form (4.9), only their initial values $\widehat{B}_{\vec{\eta}}^{i \infty}$ need to be adapted to the non-planar integration cycle.

The modified iterated Eisenstein integrals $\beta[\ldots]$ in (4.11) satisfy the differential equations

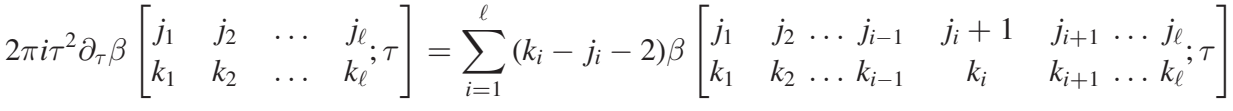

$$
\begin{aligned}
& -\delta_{j_{\ell}, k_{\ell}-2} \tau^{k_{\ell}} G_{k_{\ell}}(\tau) \beta\left[\begin{array}{llll}
j_{1} & j_{2} & \ldots & j_{\ell-1} ; \tau \\
k_{1} & k_{2} & \ldots & k_{\ell-1}
\end{array}\right] \text {, }
\end{aligned}
$$

which allows us to directly check that (4.9) obeys (3.29). The integrals $\beta[\ldots]$ inherit the property that they vanish for $\tau \rightarrow i \infty$ from the $\mathcal{E}[\ldots]$. Note that the definition (4.11) is equivalent to integral representations such as

$$
\begin{aligned}
\beta\left[\begin{array}{l}
j_{1} \\
k_{1}
\end{array} \tau\right]= & -\frac{(2 \pi i)^{1+j_{1}-k_{1}}}{\tau^{k_{1}-j_{1}-2}} \int_{i \infty}^{\tau} \mathrm{d} \tau_{1} G_{k_{1}}\left(\tau_{1}\right)\left(\tau-\tau_{1}\right)^{k_{1}-j_{1}-2} \tau_{1}^{j_{1}} \\
\beta\left[\begin{array}{ll}
j_{1} & j_{2} \\
k_{1} & k_{2}
\end{array}\right]= & \frac{(2 \pi i)^{2+j_{1}+j_{2}-k_{1}-k_{2}}}{\tau^{k_{1}+k_{2}-j_{1}-j_{2}-4}} \int_{i \infty}^{\tau} \mathrm{d} \tau_{2} G_{k_{2}}\left(\tau_{2}\right)\left(\tau-\tau_{2}\right)^{k_{2}-j_{2}-2} \tau_{2}^{j_{2}} \\
& \times \int_{i \infty}^{\tau_{2}} \mathrm{~d} \tau_{1} G_{k_{1}}\left(\tau_{1}\right)\left(\tau-\tau_{1}\right)^{k_{1}-j_{1}-2} \tau_{1}^{j_{1}} .
\end{aligned}
$$

The definition (4.11) of the $\beta[\ldots]$ preserves the shuffle relations of the iterated Eisenstein integrals (4.1), for instance

\footnotetext{
${ }^{20}$ On top of the modular $S$ transformation relating $Z_{\vec{\eta}}^{\tau}$ and $B_{\vec{\eta}}^{\tau}$, the $\mathcal{E}[\ldots]$ in (4.8) involve integration kernels $\tau^{j} G_{k}$ with $0 \leqslant j \leqslant k-2$ instead of the $G_{0}^{0}=-1$ in $[59,60]$. In other words, the relations (4.3) in the derivation algebra are built into (4.8), whereas the results in the references may require the use of shuffle relations to manifest the absence of $\mathcal{E}\left[\begin{array}{c}j \geqslant k-1 \\ k\end{array}\right]$.
} 


$$
\begin{aligned}
& \mathcal{E}\left[\begin{array}{l}
j_{1} \\
k_{1}
\end{array} \tau\right] \mathcal{E}\left[\begin{array}{l}
j_{2} \\
k_{2}
\end{array} \tau\right]=\mathcal{E}\left[\begin{array}{ll}
j_{1} & j_{2} \\
k_{1} & k_{2}
\end{array} ;\right]+\mathcal{E}\left[\begin{array}{ll}
j_{2} & j_{1} \\
k_{2} & k_{1}
\end{array} ; \tau\right] \Rightarrow \beta\left[\begin{array}{l}
j_{1} \\
k_{1}
\end{array} ;\right] \beta\left[\begin{array}{l}
j_{2} \\
k_{2}
\end{array} \tau\right] \\
& =\beta\left[\begin{array}{ll}
j_{1} & j_{2} \\
k_{1} & k_{2}
\end{array} \tau\right]+\beta\left[\begin{array}{ll}
j_{2} & j_{1} \\
k_{2} & k_{1}
\end{array} ; \tau\right] \text {. }
\end{aligned}
$$

\subsection{The $\alpha^{\prime}$-expansion of $J_{\vec{\eta}}^{\tau}$}

One can extend the above strategy to expand $B_{\vec{\eta}}^{\tau}$ via (4.4) to the $J_{\vec{\eta}}^{\tau}$ integrals. The idea is to solve their differential equation (4.5) order by order in $\alpha^{\prime}$ via

$2 \pi i \partial_{\tau} \mathcal{E}^{\mathrm{sv}}\left[\begin{array}{llll}j_{1} & j_{2} & \ldots & j_{\ell} \\ k_{1} & k_{2} & \ldots & k_{\ell}\end{array}\right]=-(2 \pi i)^{2-k_{\ell}+j_{\ell}}(\tau-\bar{\tau})^{j_{\ell}} G_{k_{\ell}}(\tau) \mathcal{E}^{\mathrm{sv}}\left[\begin{array}{llll}j_{1} & j_{2} & \ldots & j_{\ell-1} \\ k_{1} & k_{2} & \ldots & k_{\ell-1}\end{array}\right]$,

using the combinations $\mathcal{E}^{\mathrm{sv}}$ of holomorphic iterated Eisenstein integrals (4.1) and their complex conjugates introduced in [18]. Their depth $\ell=1$ instances are completely known from the reference

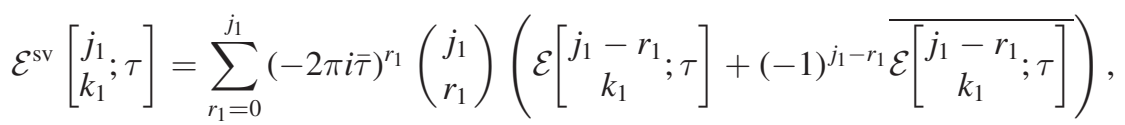

and their generalizations to depth $\ell \geqslant 2$ involve antiholomorphic integration constants $\overline{\alpha[\ldots]}$,

$$
\begin{aligned}
& \mathcal{E}^{\mathrm{sv}}\left[\begin{array}{ll}
j_{1} & j_{2} \\
k_{1} & k_{2}
\end{array} \tau\right]=\sum_{r_{1}=0}^{j_{1}} \sum_{r_{2}=0}^{j_{2}}(-2 \pi i \bar{\tau})^{r_{1}+r_{2}}\left(\begin{array}{l}
j_{1} \\
r_{1}
\end{array}\right)\left(\begin{array}{l}
j_{2} \\
r_{2}
\end{array}\right) \\
& \times\left\{\mathcal{E}\left[\begin{array}{cc}
j_{1}-r_{1} & j_{2}-r_{2} \\
k_{1} & k_{2}
\end{array} \tau\right]+(-1)^{j_{1}-r_{1}} \overline{\mathcal{E}} \overline{\left[\begin{array}{c}
j_{1}-r_{1} \\
k_{1}
\end{array} \tau\right]} \mathcal{E}\left[\begin{array}{c}
j_{2}-r_{2} \\
k_{2}
\end{array}\right]\right. \\
& \left.+(-1)^{j_{1}+j_{2}-r_{1}-r_{2}} \overline{\mathcal{E}} \overline{\left.\left[\begin{array}{cc}
j_{2}-r_{2} & j_{1}-r_{1} \\
k_{2} & k_{1}
\end{array}\right]\right]}\right\}+\overline{\left.\alpha\left[\begin{array}{ll}
j_{1} & j_{2} \\
k_{1} & k_{2}
\end{array}\right]\right]} .
\end{aligned}
$$

The integration constants $\overline{\alpha[\ldots]}$ are invariant under $\tau \rightarrow \tau+1$ since the $\mathcal{E}^{\mathrm{sv}}[\ldots]$ and the contributions from the $\mathcal{E}[\ldots], \overline{\mathcal{E}[\ldots]}$ to (4.17) are. They are known on a case-by-case basis, for instance

$$
\begin{aligned}
& \alpha\left[\begin{array}{ll}
1 & 0 \\
4 & 4
\end{array}\right]=\alpha\left[\begin{array}{ll}
0 & 1 \\
4 & 4
\end{array}\right]=0 \\
& \alpha\left[\begin{array}{ll}
2 & 0 \\
4 & 4
\end{array}\right]=\frac{2 \zeta_{3}}{3}\left(\mathcal{E}\left[\begin{array}{l}
0 \\
4
\end{array}\right]+\frac{i \pi \tau}{360}\right)=-\alpha\left[\begin{array}{ll}
0 & 2 \\
4 & 4
\end{array}\right], \\
& \alpha\left[\begin{array}{ll}
2 & 1 \\
4 & 4
\end{array}\right]=\frac{2 \zeta_{3}}{3}\left(2 \pi i \tau \mathcal{E}\left[\begin{array}{l}
0 \\
4
\end{array}\right]-\mathcal{E}\left[\begin{array}{l}
1 \\
4
\end{array}\right]-\frac{\pi^{2} \tau^{2}}{360}\right)=-\alpha\left[\begin{array}{ll}
1 & 2 \\
4 & 4
\end{array}\right],
\end{aligned}
$$

and the complete list of $\overline{\alpha\left[\begin{array}{ll}j_{1} & j_{2} \\ k_{1} & k_{2}\end{array}\right]}$ at $k_{1}+k_{2} \leqslant 12$ can be found in an ancillary file on the journal website of this work. The integration constants at arbitrary depth can be determined 
from the reality properties of the $Y_{\vec{\eta}}^{\tau}$ integrals [18]. The method in the reference to fix the $\overline{\alpha[\ldots]}$ hinges on the fact that the coefficients in the $\eta_{j}$ - and $\bar{\eta}_{j}$-expansion of $Y_{\vec{\eta}}^{\tau}$ are closed under complex conjugation. For the $n$-point $J_{\vec{\eta}}^{\tau}$-series in turn the antiholomorphic modular weights $\bar{w}$ of the integrands $\overline{V(\ldots)}$ in (3.13) are bounded by $\bar{w} \leqslant n-2$, so the complex conjugates of higher orders in the $\eta_{j}$-expansion are not part of the series. Hence, in the present formulation, the expansion of the $Y_{\vec{\eta}}^{\tau}$ in [18] is a necessary input to obtain well-defined $\mathcal{E}^{\mathrm{sv}}$. This expansion depends on the knowledge of the initial values of $Y_{\vec{\eta}}^{\tau}$ which is currently available from sphere integrals to arbitrary weight only for two points and is under investigation for higher multiplicity [95]. Still, the torus-integral- and lattice-sum representations of single-valued eMZVs in section 5.7 do not require any knowledge of $Y_{\vec{\eta}}^{i \infty}$ and $\overline{\alpha[\ldots]}$.

By repeating the steps toward (4.8) and (4.9), we arrive at the structure of the $\alpha^{\prime}$-expansion

$$
\begin{aligned}
J_{\vec{\eta}}^{\tau}= & \sum_{\ell=0}^{\infty} \sum_{\substack{k_{1}, k_{2}, \ldots, k_{\ell} \\
=4,6,6, \ldots}} \sum_{j_{1}=0}^{k_{1}-2} \sum_{j_{2}=0}^{k_{2}-2} \ldots \sum_{j_{\ell}=0}^{k_{\ell}-2}\left(\prod_{i=1}^{\ell} \frac{(-1)^{j_{i}}\left(k_{i}-1\right)}{\left(k_{i}-j_{i}-2\right) !}\right) \beta^{\mathrm{sv}}\left[\begin{array}{cccc}
j_{1} & j_{2} & \ldots & j_{\ell} \\
k_{1} & k_{2} & \ldots & k_{\ell}
\end{array}\right] \\
& \times r_{\vec{\eta}}\left(\operatorname{ad}_{\epsilon_{0}}^{k_{\ell}-j_{\ell}-2}\left(\epsilon_{k_{\ell}}\right) \ldots \operatorname{ad}_{\epsilon_{0}}^{k_{2}-j_{2}-2}\left(\epsilon_{k_{2}}\right) \operatorname{ad}_{\epsilon_{0}}^{k_{1}-j_{1}-2}\left(\epsilon_{k_{1}}\right)\right) \exp \left(-\frac{\operatorname{sv} r_{\vec{\eta}}\left(\epsilon_{0}\right)}{4 y}\right) \widehat{J}_{\vec{\eta}}^{i \infty}
\end{aligned}
$$

with an initial value $\widehat{J}_{\vec{\eta}}^{i \infty}$ to be discussed below and the combinations analogous to (4.11) [18]

$$
\begin{aligned}
\beta^{\mathrm{sv}}\left[\begin{array}{llll}
j_{1} & j_{2} & \ldots & j_{\ell} \\
k_{1} & k_{2} & \ldots & k_{\ell}
\end{array}\right]= & \sum_{p_{1}=0}^{k_{1}-j_{1}-2} \sum_{p_{2}=0}^{k_{2}-j_{2}-2} \ldots \sum_{p_{\ell}=0}^{k_{\ell}-j_{\ell}-2}\left(\begin{array}{c}
k_{1}-j_{1}-2 \\
p_{1}
\end{array}\right) \\
& \times\left(\begin{array}{c}
k_{2}-j_{2}-2 \\
p_{2}
\end{array}\right) \ldots\left(\begin{array}{c}
k_{\ell}-j_{\ell}-2 \\
p_{\ell}
\end{array}\right)\left(\frac{1}{4 y}\right)^{p_{1}+p_{2}+\cdots+p_{\ell}} \\
& \times \mathcal{E}^{\mathrm{sv}}\left[\begin{array}{cccc}
j_{1}+p_{1} & j_{2}+p_{2} & \ldots & j_{\ell}+p_{\ell} \\
k_{1} & k_{2} & \ldots & k_{\ell}
\end{array}\right] .
\end{aligned}
$$

The expansion (4.19) solves (3.30) since the $\beta^{\mathrm{sv}}$ inherit their differential equation from (4.15),

$$
\begin{aligned}
& 2 \pi i(\tau-\bar{\tau})^{2} \partial_{\tau} \beta^{\mathrm{sv}}\left[\begin{array}{llll}
j_{1} & j_{2} & \ldots & j_{\ell} \\
k_{1} & k_{2} & \ldots & k_{\ell}
\end{array}\right] \\
& =\sum_{i=1}^{\ell}\left(k_{i}-j_{i}-2\right) \beta^{\mathrm{sv}}\left[\begin{array}{cccccccc}
j_{1} & j_{2} & \ldots & j_{i-1} & j_{i}+1 & j_{i+1} & \ldots & j_{\ell} \\
k_{1} & k_{2} & \ldots & k_{i-1} & k_{i} & k_{i+1} & \ldots & k_{\ell}
\end{array}\right] \\
& -\delta_{j_{\ell}, k_{\ell}-2}(\tau-\bar{\tau})^{k_{\ell}} G_{k_{\ell}}(\tau) \beta^{\mathrm{sv}}\left[\begin{array}{llll}
j_{1} & j_{2} & \ldots & j_{\ell-1} \\
k_{1} & k_{2} & \ldots & k_{\ell-1}
\end{array}\right] \text {, }
\end{aligned}
$$

see (4.12) for the holomorphic counterpart for $\partial_{\tau} \beta[\ldots]$. Both the $\mathcal{E}^{\mathrm{sv}}[\ldots]$ and the $\beta^{\mathrm{sv}}[\ldots]$ are expected to preserve the shuffle multiplication of their holomorphic counterparts (4.1) and (4.11): the differential equations (4.15) and (4.20) recursively imply that shuffle relations among $\mathcal{E}^{\mathrm{sv}}[\ldots]$ and the $\beta^{\mathrm{sv}}[\ldots]$ can at most be violated by antiholomorphic functions such 
as the integration constants $\overline{\alpha[\ldots]}$ in (4.17). ${ }^{21}$ All examples of $\alpha\left[\begin{array}{ll}j_{1} & j_{2} \\ k_{1} & k_{2}\end{array}\right]$ up to including $k_{1}+k_{2}=12$ were checked to preserve the shuffle relations, and their explicit form can also be found in an ancillary file to the arXiv submission of this work. Note that these checks cover the more intricate cases with $\left(k_{1}, k_{2}\right)=(4,6)$ and $\left(k_{1}, k_{2}\right)=(4,8)$ where imaginary cusp forms occur among the MGFs [18, 62].

The $\mathcal{E}^{\text {sv }}$ and $\beta^{\text {sv }}$ are expected to occur in Brown's generating series of single-valued iterated Eisenstein integrals $[13,14,101]$. The construction of non-holomorphic modular forms in the references-so-called equivariant iterated Eisenstein integrals-are obtained by augmenting their single-valued counterparts by combinations of MZVs and objects of lower depth. At depth one, the equivariant iterated Eisenstein integrals are non-holomorphic Eisenstein series along with their Cauchy-Riemann derivatives [13, 14, 101]. From their representation [18]

$$
\begin{aligned}
(\pi \nabla)^{m} E_{k} & =\left(-\frac{1}{4}\right)^{m} \frac{(2 k-1) !}{(k-1) !(k-1-m) !}\left\{-\beta^{\mathrm{sv}}\left[\begin{array}{c}
k-1+m \\
2 k
\end{array}\right]+\frac{2 \zeta_{2 k-1}}{(2 k-1)(4 y)^{k-1-m}}\right\} \\
E_{k} & =\frac{(2 k-1) !}{[(k-1) !]^{2}}\left\{-\beta^{\mathrm{sv}}\left[\begin{array}{c}
k-1 \\
2 k
\end{array}\right]+\frac{2 \zeta_{2 k-1}}{(2 k-1)(4 y)^{k-1}}\right\} \\
\frac{(\pi \bar{\nabla})^{m} E_{k}}{y^{2 m}} & =\frac{(-4)^{m}(2 k-1) !}{(k-1) !(k-1-m) !}\left\{-\beta^{\mathrm{sv}}\left[\begin{array}{c}
k-1-m \\
2 k
\end{array}\right]+\frac{2 \zeta_{2 k-1}}{(2 k-1)(4 y)^{k-1+m}}\right\},
\end{aligned}
$$

the $\beta^{\text {sv }}$ are seen to take the role of the single-valued rather than equivariant iterated Eisenstein integrals at depth one. At higher depth, the precise relation of the $\beta^{\mathrm{sv}}$ to Brown's construction is an open question at the time of writing.

\subsection{Initial values}

It remains to specify the initial values $\widehat{B}_{\vec{\eta}}^{i \infty}$ and $\widehat{J}_{\vec{\eta}}^{i \infty}$ in the $\alpha^{\prime}$-expansions (4.9) and (4.19). The Laurent-polynomial contributions from the asymptotics (3.24) and (3.25) of the Green functions are still functions of $\tau$ and need to be translated into series that solely depend on $\eta_{j}$ and $s_{i j}$. Following the construction of a similar initial value for $Y_{\vec{\eta}}^{\tau}$ in section 3.4 of [18], we import the constant parts $\sim \tau^{0}$ and $\sim(\operatorname{Im} \tau)^{0}$ of the respective Laurent polynomials

$$
\begin{aligned}
& \widehat{B}_{\vec{\eta}}^{i \infty}=\left.\left.\exp \left(\frac{i r_{\vec{\eta}}\left(\epsilon_{0}\right)}{2 T}\right) B_{\vec{\eta}}^{\tau}\right|_{\mathrm{LP}}\right|_{\tau^{0}} \\
& \widehat{J}_{\vec{\eta}}^{i \infty}=\left.\left.\exp \left(\frac{\operatorname{sv} r_{\vec{\eta}}\left(\epsilon_{0}\right)}{4 y}\right) J_{\vec{\eta}}^{\tau}\right|_{\mathrm{LP}}\right|_{(\operatorname{Im} \tau)^{0}} .
\end{aligned}
$$

In both cases, the exponentials ensure that the negative powers of $T$ in $\left.B_{\vec{\eta}}^{\tau}\right|_{\mathrm{LP}}$ and $y$ in $\left.J_{\vec{\eta}}^{\tau}\right|_{\mathrm{LP}}$ disappear order by order in $\alpha^{\prime}$. Hence, (4.23) and (4.24) pick up the lowest powers of $T, y$ present in $\left.\exp \left(\frac{i r_{\vec{r}}\left(\epsilon_{0}\right)}{2 T}\right) B_{\vec{\eta}}^{\tau}\right|_{\mathrm{LP}}$ and $\left.\exp \left(\frac{\mathrm{sv} r_{\vec{r}}\left(\epsilon_{0}\right)}{4 y}\right) J_{\vec{\eta}}^{\tau}\right|_{\mathrm{LP}}$. The leading $\alpha^{\prime}$ - and $\eta_{2}$-orders of the

\footnotetext{
${ }^{21}$ Moreover, any such violation of shuffle relations would need to be a combination of antiholomorphic iterated Eisenstein integrals (by the differential equation [18, (2.37)] for $\partial_{\bar{\tau}} Y_{\vec{\eta}}^{\tau}$ ) but at the same time line up with the modular weights in the $\eta_{j}$-expansion of $J_{\vec{\eta}}^{\tau}, Y_{\vec{\eta}}^{\tau}$ and the reality properties of the latter. It would be interesting to find a rigorous argument to rule out the existence of such antiholomorphic functions.
} 
two-point initial values following from the expressions in (3.26) and (3.27) are

$$
\begin{aligned}
\widehat{B}_{\eta_{2}}^{i \infty}= & \frac{1}{\eta_{2}}\left[1+\frac{1}{6} s_{12}^{2} \zeta_{2}+\frac{1}{12} s_{12}^{3} \zeta_{3}+\frac{131}{720} s_{12}^{4} \zeta_{4}+s_{12}^{5}\left(\frac{17}{360} \zeta_{2} \zeta_{3}+\frac{43 \zeta_{5}}{720}\right)+\mathcal{O}\left(s_{12}^{6}\right)\right] \\
& +\eta_{2}\left[-2 \zeta_{2}-s_{12} \zeta_{3}-\frac{29}{12} s_{12}^{2} \zeta_{4}-s_{12}^{3}\left(\frac{1}{3} \zeta_{2} \zeta_{3}+\frac{5 \zeta_{5}}{6}\right)\right. \\
& \left.-s_{12}^{4}\left(\frac{1}{12} \zeta_{3}^{2}+\frac{87 \zeta_{6}}{40}\right)+\mathcal{O}\left(s_{12}^{5}\right)\right] \\
& +\eta_{2}^{3}\left[-2 \zeta_{4}+s_{12}\left(2 \zeta_{2} \zeta_{3}-\zeta_{5}\right)+s_{12}^{2}\left(\frac{\zeta_{3}^{2}}{2}-\frac{33 \zeta_{6}}{8}\right)\right. \\
& \left.+s_{12}^{3}\left(\frac{9}{4} \zeta_{3} \zeta_{4}+\frac{3}{2} \zeta_{2} \zeta_{5}-\frac{7 \zeta_{7}}{4}\right)+\mathcal{O}\left(s_{12}^{4}\right)\right] \\
& +\eta_{2}^{5}\left[-2 \zeta_{6}+s_{12}\left(2 \zeta_{4} \zeta_{3}+2 \zeta_{2} \zeta_{5}-\zeta_{7}\right)+s_{12}^{2}\left(-\zeta_{2} \zeta_{3}^{2}+\zeta_{3} \zeta_{5}-\frac{43 \zeta_{8}}{6}\right)+\mathcal{O}\left(s_{12}^{3}\right)\right] \\
& +\eta_{2}^{7}\left[-2 \zeta_{8}+s_{12}\left(2 \zeta_{3} \zeta_{6}+2 \zeta_{4} \zeta_{5}+2 \zeta_{2} \zeta_{7}-\zeta_{9}\right)+\mathcal{O}\left(s_{12}^{2}\right)\right] \\
& +\eta_{2}^{9}\left[-2 \zeta_{10}+\mathcal{O}\left(s_{12}\right)\right]+\mathcal{O}\left(\eta_{2}^{11}\right)
\end{aligned}
$$

as well as

$$
\begin{aligned}
\widehat{J}_{\eta_{2}}^{i \infty}= & \frac{1}{\eta_{2}}\left[1+\frac{1}{6} s_{12}^{3} \zeta_{3}+\frac{43}{360} s_{12}^{5} \zeta_{5}+\mathcal{O}\left(s_{12}^{6}\right)\right] \\
& +\eta_{2}\left[-2 s_{12} \zeta_{3}-\frac{5}{3} s_{12}^{3} \zeta_{5}-\frac{1}{3} s_{12}^{4} \zeta_{3}^{2}+\mathcal{O}\left(s_{12}^{5}\right)\right] \\
& +\eta_{2}^{3}\left[-2 s_{12} \zeta_{5}+2 s_{12}^{2} \zeta_{3}^{2}-\frac{7}{2} s_{12}^{3} \zeta_{7}+\mathcal{O}\left(s_{12}^{4}\right)\right] \\
& +\eta_{2}^{5}\left[-2 s_{12} \zeta_{7}+4 s_{12}^{2} \zeta_{3} \zeta_{5}+\mathcal{O}\left(s_{12}^{3}\right)\right] \\
& +\eta_{2}^{7}\left[-2 s_{12} \zeta_{9}+\mathcal{O}\left(s_{12}^{2}\right)\right]+\eta_{2}^{9} \mathcal{O}\left(s_{12}\right)+\mathcal{O}\left(\eta_{2}^{11}\right) .
\end{aligned}
$$

Higher orders in $s_{12}$ and $\eta_{2}$ are readily available through the straightforward expansion of the exponentials and $\Gamma$-functions in (3.26) and (3.27). In particular, these two-point expressions imply that all the coefficients in the $s_{12}$ - and $\eta_{2}$-expansions are combinations of Riemann zeta values for $\widehat{B}_{\eta_{2}}^{i \infty}$ and odd Riemann zeta values for $\widehat{J}_{\eta_{2}}^{i \infty}$.

Starting from $n=3$ points, the initial values $\widehat{B}_{\vec{\eta}}^{i \infty}$ will also feature irreducible MZVs of higher depth. Based on Enriquez' method to generate the Laurent polynomial of B-cycle eMZVs [3] (also see appendix B of [51]) we have determined the three-point initial values to certain orders, and the results are included in an ancillary file to the arXiv submission of this article. To the orders under consideration, we find the following coefficients of $\zeta_{3,5}$

$$
\begin{aligned}
\left.\widehat{B}_{\eta_{2}, \eta_{3}}^{i \infty}(2,3 \mid 2,3)\right|_{\zeta_{3,5}}= & \frac{1}{10}\left(\eta_{23}-2 \eta_{3}\right)\left(2 \eta_{23}-\eta_{3}\right)\left(\eta_{23}+\eta_{3}\right) \\
& \times\left[2 \eta_{23}^{2} s_{13}-2 \eta_{23} \eta_{3} s_{12}-4 \eta_{23} \eta_{3} s_{13}+2 \eta_{3}^{2} s_{13}\right. \\
& \left.-2 \eta_{23} \eta_{3} s_{23}+\eta_{23} s_{12} s_{13}+\eta_{3} s_{13} s_{23}+\mathcal{O}\left(s_{i j}^{3}\right)\right]
\end{aligned}
$$




$$
\begin{aligned}
\left.\widehat{B}_{\eta_{2}, \eta_{3}}^{i \infty}(2,3 \mid 3,2)\right|_{\zeta_{3,5}}= & -\frac{1}{10}\left(\eta_{2}-2 \eta_{23}\right)\left(2 \eta_{2}-\eta_{23}\right)\left(\eta_{2}+\eta_{23}\right) \\
& \times\left[2 \eta_{2}^{2} s_{12}-4 \eta_{2} \eta_{23} s_{12}+2 \eta_{23}^{2} s_{12}-2 \eta_{2} \eta_{23} s_{13}\right. \\
& \left.-2 \eta_{2} \eta_{23} s_{23}-\eta_{23} s_{12} s_{13}-\eta_{2} s_{12} s_{23}+\mathcal{O}\left(s_{i j}^{3}\right)\right],
\end{aligned}
$$

which by the single-valued maps sv $\zeta_{3,5}=-10 \zeta_{3} \zeta_{5}$ and sv $\zeta_{3} \zeta_{5}=4 \zeta_{3} \zeta_{5}$ enter the closedstring initial values via

$$
\begin{aligned}
\left.\widehat{\boldsymbol{J}}_{\eta_{2}, \eta_{3}}^{i \infty}(2,3 \mid \rho(2,3))\right|_{\zeta_{3} \zeta_{5}}= & -\left.10 \widehat{B}_{\eta_{2}, \eta_{3}}^{i \infty}(2,3 \mid \rho(2,3))\right|_{\zeta_{3,5}} \\
& +\left.4 \widehat{B}_{\eta_{2}, \eta_{3}}^{i \infty}(2,3 \mid \rho(2,3))\right|_{\zeta_{3} \zeta_{5}} .
\end{aligned}
$$

The coefficients of $\zeta_{3,5}$ and $\zeta_{3} \zeta_{5}$ in (4.27) and (4.28) are extracted after reducing all MZVs at weight 8 to $\mathbb{Q}$-linear combinations of $\left\{\zeta_{8}, \zeta_{2} \zeta_{3}^{2}, \zeta_{3} \zeta_{5}, \zeta_{3,5}\right\}$ [102]. Similarly, the MZV $\zeta_{3,5,3}$ seen in Laurent polynomials of both B-cycle integrals [51] and modular graph functions [12] will occur in both $\widehat{B}_{\eta_{2}, \eta_{3}}^{i \infty}$ and $\widehat{J}_{\eta_{2}, \eta_{3}}^{i \infty}$. The contributions to $\widehat{B}_{\eta_{2}, \eta_{3}}^{i \infty}$ involving MZVs of weight up to and including four can be found in appendix B.

Since the initial values are obtained from the Laurent polynomials and the exponents in (4.23) and (4.24) are related by the single-valued map, the conjecture (3.23) supported by tree-level results and extensive genus-one tests is equivalent to

$$
\widehat{J}_{\vec{\eta}}^{i \infty}(\gamma \mid \rho)=\operatorname{sv} \widehat{B}_{\vec{\eta}}^{i \infty}(\gamma \mid \rho)
$$

in agreement with (4.25) and (4.26).

The $\tau \rightarrow i \infty$ asymptotics of $n$-point A-cycle integrals (2.9) has been expressed in terms of $(n+2)$-point disk integrals $(2.1)$ in suitable kinematic limits [60]. Similarly, the Laurent polynomials of $n$-point genus-one integrals $B_{\vec{\eta}}^{\tau}, J_{\vec{\eta}}^{\tau}$ are determined by genus-zero integrals at multiplicity $n+2$ and below, see (3.26) and (3.27) for the explicit two-point result. As will be further investigated in [95], the main challenge is to determine the admixture of lowerpoint genus-zero integrals that generalize the subtraction of $\mathrm{e}^{i T\left(\frac{s_{12}}{2} \pm \eta_{2}\right)}$ and $\mathrm{e}^{-y\left(s_{12} \pm 2 \eta_{2}\right)}$ from the $\Gamma$-functions in (3.26) and (3.27).

\subsection{The single-valued map on iterated Eisenstein integrals}

The proposed single-valued map (3.35) can now also be studied at the level of the $\alpha^{\prime}$ expansions. Using (4.29) and (3.31), we find that one obtains $J_{\vec{\eta}}^{\tau}$ as the single-valued version of $B_{\vec{\eta}}^{\tau}$ if the coefficients obey

$$
\left.\beta^{\mathrm{sv}}\left[\begin{array}{l}
j_{1} j_{2} \ldots j_{\ell} \\
k_{1} k_{2} \ldots k_{\ell}
\end{array}\right]=\mathrm{SV} \beta\left[\begin{array}{l}
j_{1} j_{2} \ldots j_{\ell} \\
k_{1} k_{2} \ldots k_{\ell}
\end{array}\right] \tau\right]
$$

This follows from the relation (4.29) among the initial values and the form of the $r_{\vec{\eta}}$ operators in the respective $\alpha^{\prime}$-expansions, recalling that $r_{\vec{\eta}}\left(\operatorname{ad}_{\epsilon_{0}}^{j}\left(\epsilon_{k}\right)\right)=\operatorname{sv} r_{\vec{\eta}}\left(\operatorname{ad}_{\epsilon_{0}}^{j}\left(\epsilon_{k}\right)\right)$.

On the one hand, (4.30) fixes the single-valued map of the eMZVs in the expansion of $B_{\vec{\eta}}^{\tau}$ that enter through the iterated Eisenstein integrals $\beta[\ldots]$. On the other hand, (4.30) only applies to the combinations $\beta[\ldots]$ and $\beta^{\mathrm{sv}}[\ldots]$ that occur in the path-ordered exponentials (4.9) and (4.19). The SV map of individual $\beta\left[\begin{array}{ccc}j_{1} & \ldots & j_{\ell} \\ k_{1} & \ldots & k_{\ell}\end{array} \tau\right]$ remains undetermined whenever the relations in the derivation algebra such as $(2.22)$ lead to dropouts of certain $\beta[\ldots]$ and $\beta^{\mathrm{sv}}[\ldots]$ from $B_{\vec{\eta}}^{\tau}$ and $J_{\vec{\eta}}^{\tau}$ (starting with cases at $\left(k_{1}, k_{2}\right)=(10,4)$ at depth $\ell=2$ and any instance where $\left.j_{i}>k_{i}-2\right)$. 
Since the factors of $\frac{i}{2 T}$ and $\frac{1}{4 y}$ in (4.11) and (4.20) are furthermore related by SV, (4.30) is equivalent to

$$
\mathcal{E}^{\mathrm{sv}}\left[\begin{array}{l}
j_{1} j_{2} \ldots j_{\ell} \\
k_{1} k_{2} \ldots k_{\ell} ; \tau
\end{array}\right]=\operatorname{SV} \mathcal{E}\left[\begin{array}{l}
j_{1} j_{2} \ldots j_{\ell} \\
k_{1} k_{2} \ldots k_{\ell}
\end{array} ; \tau\right]
$$

again up to cases where the relations in the derivation algebra cause dropouts. For instance, (4.16) implies that the single-valued version of holomorphic Eisenstein integrals (4.1) at depth one is given by

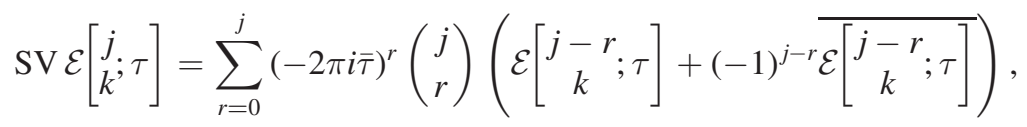

where the contributions on the right-hand side can be recognized as

$$
\begin{aligned}
& \sum_{r=0}^{j}(-2 \pi i \bar{\tau})^{r}\left(\begin{array}{l}
j \\
r
\end{array}\right) \mathcal{E}\left[\begin{array}{c}
j-r \\
k
\end{array} ; \tau\right]=(2 \pi i)^{1-k+j} \int_{\tau}^{i \infty} \mathrm{d} \tau_{1}\left(\tau_{1}-\bar{\tau}\right)^{j} G_{k}\left(\tau_{1}\right) \\
& (-1)^{j} \sum_{r=0}^{j}(2 \pi i \bar{\tau})^{r}\left(\begin{array}{c}
j \\
r
\end{array}\right) \overline{\mathcal{E}} \overline{\left[\begin{array}{c}
j-r \\
k
\end{array} ; \tau\right]}=-(2 \pi i)^{1-k+j} \int_{\bar{\tau}}^{i \infty} \mathrm{d} \bar{\tau}_{1}\left(\bar{\tau}_{1}-\bar{\tau}\right)^{j} \overline{G_{k}\left(\tau_{1}\right)},
\end{aligned}
$$

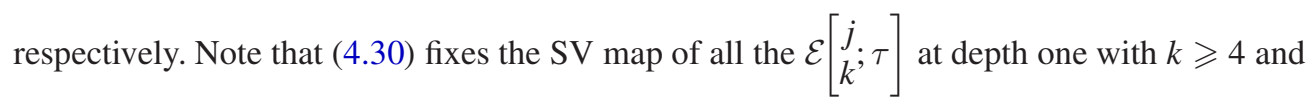
$0 \leqslant j \leqslant k-2$ since the caveats related to relations in the derivation algebra beyond (4.3) only affect iterated Eisenstein integrals of depth $\ell \geqslant 2$.

The iterated Eisenstein integrals defined in (4.1) may be reorganized in terms of [17]

$$
\mathcal{E}_{0}\left(k_{1}, k_{2}, \ldots, k_{\ell} ; \tau\right)=2 \pi i \int_{\tau}^{i \infty} \mathrm{d} \tau_{\ell} \frac{G_{k_{\ell}}^{0}\left(\tau_{\ell}\right)}{(2 \pi i)^{k_{\ell}}} \mathcal{E}_{0}\left(k_{1}, k_{2}, \ldots, k_{\ell-1} ; \tau\right)
$$

with $k_{i} \in 2 \mathbb{N}_{0}$ and $\mathcal{E}_{0}(; \tau)=1$. By subtracting the zero mode of the holomorphic Eisenstein series

$$
\begin{aligned}
& G_{k}^{0}(\tau)=G_{k}(\tau)-2 \zeta_{k} \quad \text { for } k>0 \text { even } \\
& G_{0}^{0}(\tau)=G_{0}(\tau)=-1
\end{aligned}
$$

the integrals (4.34) are made to converge if $k_{1}>0$, and all other cases are shuffle-regularized based on $\mathcal{E}_{0}(0 ; \tau)=2 \pi i \tau$.

At depth one, they are related to the holomorphic iterated Eisenstein integrals via [51]

$$
\mathcal{E}\left[\begin{array}{l}
j \\
k
\end{array} \tau\right]=j ! \mathcal{E}_{0}\left(0^{j}, k ; \tau\right)+\frac{B_{k}(2 \pi i \tau)^{j+1}}{k !(j+1)}
$$

with Bernoulli numbers $B_{k}$. From this, we see that the implicit action of SV on these functions at depth one is given by

$$
\mathrm{SV} \mathcal{E}_{0}\left(0^{j}, k ; \tau\right)=\frac{1}{j !} \mathcal{E}^{\mathrm{sv}}\left[\begin{array}{l}
j \\
k
\end{array} \tau\right]-\frac{B_{k}(-4 y)^{j+1}}{k !(j+1) !}
$$


By (4.32), (4.36) and the shuffle relation

$$
\mathcal{E}_{0}\left(0^{j}, k ; \tau\right)=\sum_{r=0}^{j} \frac{(-1)^{j-r}}{r !}(2 \pi i \tau)^{r} \mathcal{E}_{0}\left(k, 0^{j-r} ; \tau\right),
$$

two equivalent formulations of (4.37) are

$$
\begin{aligned}
& \operatorname{SV} \mathcal{E}_{0}\left(0^{j}, k ; \tau\right)=\mathcal{E}_{0}\left(0^{j}, k ; \tau\right)+\sum_{r=1}^{j} \overline{\mathcal{E}_{0}\left(0^{r} ; \tau\right)} \mathcal{E}_{0}\left(0^{j-r}, k ; \tau\right)+\overline{\mathcal{E}_{0}\left(k, 0^{j} ; \tau\right)} \\
& \operatorname{SV} \mathcal{E}_{0}\left(k, 0^{j} ; \tau\right)=\mathcal{E}_{0}\left(k, 0^{j} ; \tau\right)+\sum_{r=1}^{j} \overline{\mathcal{E}_{0}\left(0^{j-r}, k ; \tau\right)} \mathcal{E}_{0}\left(0^{r} ; \tau\right)+\overline{\mathcal{E}_{0}\left(0^{j}, k ; \tau\right)},
\end{aligned}
$$

which match the expectation from [103].

\section{Examples}

We shall now spell out a variety of examples that illustrate both the pairing of cycles with dual antielliptic integrands $\overline{V(\ldots \mid \tau)}$ and the action of the single-valued map on eMZVs. Both eMZVs and MGFs occur in the simultaneous expansion of the generating series $B_{\vec{\eta}}^{\tau}, J_{\vec{\eta}}^{\tau}$ in $s_{i j}$ and $\eta_{j}$ which results in a lattice-sum representation of all convergent $\mathrm{SV} \omega\left(n_{1}, \ldots, n_{r} \mid-\frac{1}{\tau}\right)$ in section 5.7. The coefficients in the $\eta_{j}$-expansions will be referred to as component integrals, and we will use the shorthand

$$
f_{i j}^{(a)}=f^{(a)}\left(z_{i}-z_{j}, \tau\right), \quad \overline{f_{i j}^{(b)}}=\overline{f^{(b)}\left(z_{i}-z_{j}, \tau\right)}
$$

for the Kronecker-Eisenstein coefficients defined by (2.14) that occur in the integrands. More precisely, the building blocks (2.13) and (3.6) of the integrands of $B_{\vec{\eta}}^{\tau}, J_{\vec{\eta}}^{\tau}$ involve the following combinations of (5.1) with $\eta_{j, j+1 \ldots n}=\eta_{j}+\eta_{j+1}+\cdots+\eta_{n}$,

$$
\begin{aligned}
\varphi_{\vec{\eta}}^{\tau}(1,2, \ldots, n) & =\sum_{a_{2}, \ldots, a_{n} \geqslant 0} \eta_{23 \ldots n}^{a_{2}-1} \eta_{3 \ldots n}^{a_{3}-1} \ldots \eta_{n}^{a_{n}-1} f_{12}^{\left(a_{2}\right)} f_{23}^{\left(a_{3}\right)} \ldots f_{n-1, n}^{\left(a_{n}\right)} \\
V_{w}(1,2, \ldots, n \mid \tau) & =\sum_{\substack{a_{1}, a_{2}, \ldots, a_{n} \geqslant 0 \\
a_{1}+a_{2}+\cdots+a_{n}=w}} f_{12}^{\left(a_{1}\right)} f_{23}^{\left(a_{2}\right)} \ldots f_{n-1, n}^{\left(a_{n-1}\right)} f_{n, 1}^{\left(a_{n}\right)} .
\end{aligned}
$$

\subsection{Two-point $\alpha^{\prime}$-expansions}

At two points, the general definitions (3.1) and (3.13) only admit a single permutation of the integrands and cycle in $B_{\eta_{2}}^{\tau}=B_{\eta_{2}}^{\tau}(2 \mid 2)$ and $J_{\eta_{2}}^{\tau}=J_{\eta_{2}}^{\tau}(2 \mid 2)$,

$$
\begin{aligned}
B_{\eta_{2}}^{\tau} & =\int_{-\tau / 2}^{\tau / 2} \frac{\mathrm{d} z_{2}}{\tau \eta_{2}} \sum_{a=0}^{\infty} \tau^{a} \eta_{2}^{a} f_{12}^{(a)} \mathrm{e}^{s_{12} \mathcal{G}_{\mathfrak{B}}\left(z_{12}, \tau\right)} \\
J_{\eta_{2}}^{\tau} & =\int_{\mathfrak{T}} \frac{\mathrm{d}^{2} z_{2}}{\operatorname{Im} \tau} \frac{1}{\eta_{2}} \sum_{a=0}^{\infty}(\tau-\bar{\tau})^{a} \eta_{2}^{a} f_{12}^{(a)} \mathrm{e}^{s_{12} \mathcal{G}_{\mathfrak{T}}\left(z_{12}, \tau\right)},
\end{aligned}
$$


and we introduce the following notation for component integrals

$$
\begin{aligned}
& B_{(a)}^{\tau}=\left.B_{\eta_{2}}^{\tau}\right|_{\eta_{2}^{a-1}}=\tau^{a} \int_{-\tau / 2}^{\tau / 2} \frac{\mathrm{d} z_{2}}{\tau} f_{12}^{(a)} \mathrm{e}^{s_{12} \mathcal{G}_{\mathfrak{B}}\left(z_{12}, \tau\right)} \\
& \boldsymbol{J}_{(a)}^{\tau}=\left.J_{\eta_{2}}^{\tau}\right|_{\eta_{2}^{a-1}}=(\tau-\bar{\tau})^{a} \int_{\mathfrak{T}} \frac{\mathrm{d}^{2} z_{2}}{\operatorname{Im} \tau} f_{12}^{(a)} \mathrm{e}^{s_{12} \mathcal{G}_{\mathfrak{T}}\left(z_{12}, \tau\right)} .
\end{aligned}
$$

Then, combining the initial values (4.25) and (4.26) with the $\alpha^{\prime}$-expansions (4.9) and (4.19) yields expressions like

$$
\begin{aligned}
& B_{(0)}^{\tau}=1+s_{12}^{2}\left(-3 \beta\left[\frac{1}{4} ; \tau\right]+\frac{\zeta_{2}}{6}+\frac{i \zeta_{3}}{2 T}+\frac{3 \zeta_{4}}{4 T^{2}}\right) \\
& +s_{12}^{3}\left(-5 \beta\left[\begin{array}{l}
2 \\
6
\end{array} ;\right]+12 \zeta_{2} \beta\left[\begin{array}{l}
0 \\
4
\end{array} ; \tau\right]+\frac{\zeta_{3}}{12}+\frac{19 i \zeta_{4}}{24 T}-\frac{\zeta_{5}}{4 T^{2}}+\frac{\zeta_{2} \zeta_{3}}{T^{2}}-\frac{4 i \zeta_{6}}{3 T^{3}}\right) \\
& +\mathcal{O}\left(s_{12}^{4}\right) \\
& B_{(2)}^{\tau}=-2 \zeta_{2}+s_{12}\left(3 \beta\left[\begin{array}{l}
2 \\
4
\end{array} \tau\right]-\zeta_{3}+\frac{3 i \zeta_{4}}{T}\right) \\
& +s_{12}^{2}\left(10 \beta\left[\begin{array}{l}
3 \\
6
\end{array} \tau\right]-18 \zeta_{2} \beta\left[\begin{array}{l}
1 \\
4
\end{array} \tau\right]-\frac{29 \zeta_{4}}{12}-\frac{i \zeta_{5}}{T}+\frac{3 i \zeta_{2} \zeta_{3}}{T}+\frac{43 \zeta_{6}}{8 T^{2}}\right) \\
& +\mathcal{O}\left(s_{12}^{3}\right)
\end{aligned}
$$

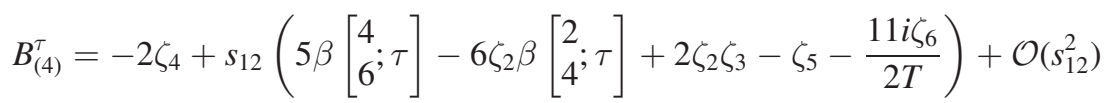

as well as

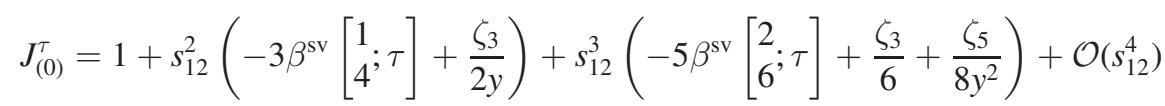

$$
\begin{aligned}
& J_{(2)}^{\tau}=s_{12}\left(3 \beta^{\mathrm{sv}}\left[\begin{array}{l}
2 \\
4
\end{array} ;\right]-2 \zeta_{3}\right)+s_{12}^{2}\left(10 \beta^{\mathrm{sv}}\left[\begin{array}{l}
3 \\
6
\end{array} ; \tau\right]-\frac{\zeta_{5}}{y}\right)+\mathcal{O}\left(s_{12}^{3}\right) \\
& J_{(4)}^{\tau}=s_{12}\left(5 \beta^{\mathrm{sv}}\left[\begin{array}{l}
4 \\
6
\end{array} ;\right]-2 \zeta_{5}\right)+\mathcal{O}\left(s_{12}^{2}\right)
\end{aligned}
$$

upon extracting suitable powers of $\eta_{2}$. The action of SV on the $\zeta_{n}, T, \beta[\ldots]$ as in (3.33) and (4.30) relates $J_{(a)}^{\tau}=\mathrm{SV} B_{(a)}^{\tau}$ as expected from (3.35). Examples of $\beta[\ldots]$ beyond depth one occur at the next orders in $s_{i j}$, e.g.

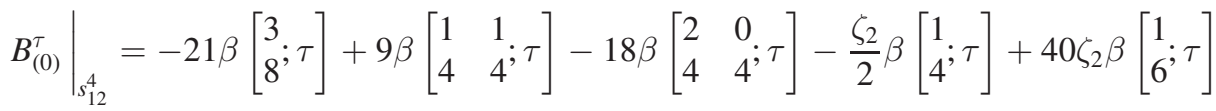

$$
\begin{aligned}
& +6 \zeta_{3} \beta\left[{ }_{4}^{0} ; \tau\right]-\frac{3 i \zeta_{3}}{2 T} \beta\left[{ }_{4}^{1} ; \tau\right]-\frac{18 i \zeta_{4}}{T} \beta\left[\begin{array}{l}
0 \\
4
\end{array} ; \tau\right]-\frac{9 \zeta_{4}}{4 T^{2}} \beta\left[{ }_{4}^{1} ; \tau\right] \\
& +\frac{131 \zeta_{4}}{720}+\frac{5 i \zeta_{5}}{12 T}+\frac{i \zeta_{2} \zeta_{3}}{12 T}+\frac{23 \zeta_{6}}{32 T^{2}}+\frac{\zeta_{3}^{2}}{8 T^{2}}-\frac{9 i \zeta_{3} \zeta_{4}}{8 T^{3}}
\end{aligned}
$$




$$
+\frac{i \zeta_{2} \zeta_{5}}{T^{3}}-\frac{3 i \zeta_{7}}{8 T^{3}}+\frac{85 \zeta_{8}}{192 T^{4}}
$$

as well as

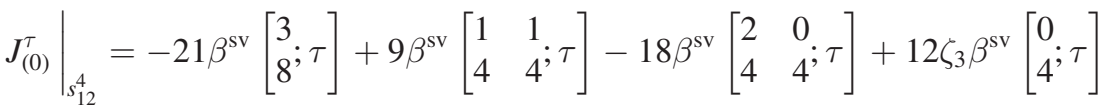

$$
\begin{aligned}
& -\frac{3 \zeta_{3}}{2 y} \beta^{\mathrm{sv}}[1 ; ; \tau]+\frac{5 \zeta_{5}}{12 y}-\frac{\zeta_{3}^{2}}{8 y^{2}}+\frac{3 \zeta_{7}}{32 y^{3}}
\end{aligned}
$$

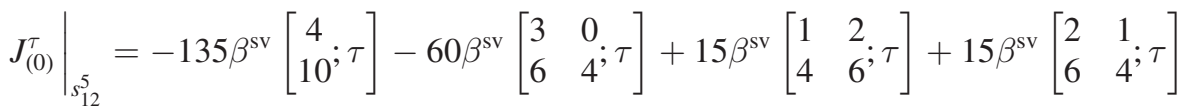

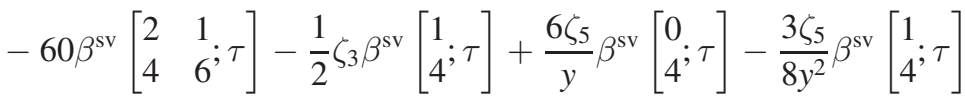

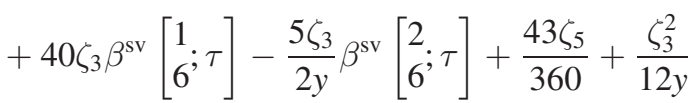

$$
\begin{aligned}
& +\frac{7 \zeta_{7}}{32 y^{2}}-\frac{3 \zeta_{3} \zeta_{5}}{16 y^{3}}+\frac{15 \zeta_{9}}{128 y^{4}}
\end{aligned}
$$

\subsection{Extracting single-valued eMZVs}

The above $\alpha^{\prime}$-expansions at two points have been generated in earlier work in terms of eMZVs $[51,59]$ and MGFs $[10,11,61]$, respectively. The results in the references include

$$
\begin{aligned}
B_{(0)}^{\tau}= & +s_{12}^{2}\left(\frac{1}{2} \omega\left(0,0,2 \mid-\frac{1}{\tau}\right)+\frac{5 \zeta_{2}}{12}\right) \\
& +s_{12}^{3}\left(\frac{1}{18} \omega\left(0,0,3,0 \mid-\frac{1}{\tau}\right)-\frac{4}{3} \zeta_{2} \omega\left(0,0,1,0 \mid-\frac{1}{\tau}\right)+\frac{\zeta_{3}}{12}\right) \\
& +s_{12}^{4}\left(-\omega\left(0,0,0,2,2 \mid-\frac{1}{\tau}\right)-\frac{5}{4} \omega\left(0,0,0,0,4 \mid-\frac{1}{\tau}\right)+\frac{1}{8} \omega\left(0,0,4 \mid-\frac{1}{\tau}\right)\right. \\
& \left.+\frac{5}{8} \omega\left(0,0,2 \mid-\frac{1}{\tau}\right)^{2}+\frac{13}{24} \zeta_{2} \omega\left(0,0,2 \mid-\frac{1}{\tau}\right)-2 \zeta_{2} \omega\left(0,0,0,0,2 \mid-\frac{1}{\tau}\right)+\frac{343 \zeta_{4}}{576}\right) \\
& +\mathcal{O}\left(s_{12}^{5}\right) \\
J_{(0)}^{\tau}= & +\frac{1}{2} s_{12}^{2} E_{2}(\tau)+\frac{1}{6} s_{12}^{3}\left(E_{3}(\tau)+\zeta_{3}\right)+s_{12}^{4}\left(E_{2,2}(\tau)+\frac{3}{20} E_{4}(\tau)+\frac{1}{8} E_{2}^{2}(\tau)\right) \\
& +\mathcal{O}\left(s_{12}^{5}\right),
\end{aligned}
$$

where for instance (by comparison with (5.5) and (5.6))

$$
\omega\left(0,0,2 \mid-\frac{1}{\tau}\right)=-6 \beta\left[\frac{1}{4} ; \tau\right]-\frac{\zeta_{2}}{2}+\frac{i \zeta_{3}}{T}+\frac{3 \zeta_{4}}{2 T^{2}}, \quad E_{2}(\tau)=-6 \beta^{\mathrm{sv}}\left[\frac{1}{4} ; \tau\right]+\frac{\zeta_{3}}{y},
$$


and the modular transformation may be evaluated to yield [51, 93]

$$
\omega\left(0,0,2 \mid-\frac{1}{\tau}\right)=-\frac{T^{2}}{180}-\frac{\zeta_{2}}{6}+\frac{3 \zeta_{4}}{2 T^{2}}+\omega(0,0,2 \mid \tau)+\frac{8 i}{T} \zeta_{2} \omega(0,1,0,0 \mid \tau) .
$$

For the $s_{12}^{2}$-order in (5.9), the component version $J_{(a)}^{\tau}=\mathrm{SV} B_{(a)}^{\tau}$ of $\boldsymbol{J}_{\eta_{2}}^{\tau}=\mathrm{SV} B_{\eta_{2}}^{\tau}$ implies that

$$
\mathrm{SV} \omega\left(0,0,2 \mid-\frac{1}{\tau}\right)=E_{2}(\tau)
$$

and a similar analysis for higher orders in $s_{12}$ and at $a \neq 0$ yields for instance

$$
\begin{aligned}
\operatorname{SV} \omega\left(0,3 \mid-\frac{1}{\tau}\right)=2 \pi \nabla E_{2}(\tau), & \operatorname{SV} \omega\left(0,0,4 \mid-\frac{1}{\tau}\right)=-\frac{4}{3} \pi \nabla E_{3}(\tau) \\
\operatorname{SV} \omega\left(0,0,3,0 \mid-\frac{1}{\tau}\right)=3 E_{3}(\tau), & \operatorname{SV} \omega\left(0,5 \mid-\frac{1}{\tau}\right)=-\frac{4}{3}(\pi \nabla)^{2} E_{3}(\tau) .
\end{aligned}
$$

At depth two, relating $\left.\left.B_{(0)}^{\tau}\right|_{s_{12}^{4}} \leftrightarrow J_{(0)}^{\tau}\right|_{s_{12}^{4}}$ (see (5.7) and (5.8)) or $\left.\left.\boldsymbol{B}_{(2)}^{\tau}\right|_{s_{12}^{3}} \leftrightarrow \boldsymbol{J}_{(2)}^{\tau}\right|_{s_{12}^{3}}$ yields

$$
\begin{aligned}
E_{2,2}(\tau)= & \operatorname{SV}\left(-\frac{7}{5} \omega\left(0,0,0,0,4 \mid-\frac{1}{\tau}\right)-\omega\left(0,0,0,2,2 \mid-\frac{1}{\tau}\right)\right. \\
& \left.+\frac{1}{2} \omega\left(0,0,2 \mid-\frac{1}{\tau}\right)^{2}+\frac{3}{20} \omega\left(0,0,4 \mid-\frac{1}{\tau}\right)\right) \\
\pi \nabla E_{2,2}(\tau)= & \operatorname{SV}\left(-\frac{1}{60} \omega\left(0,5 \mid-\frac{1}{\tau}\right)+\frac{3}{5} \omega\left(0,0,0,5 \mid-\frac{1}{\tau}\right)\right. \\
& \left.-\frac{1}{2} \omega\left(0,0,2,3 \mid-\frac{1}{\tau}\right)\right),
\end{aligned}
$$

where the combinations

$$
\begin{aligned}
E_{2,2} & =\left(\frac{\operatorname{Im} \tau}{\pi}\right)^{4}\left(\mathcal{C}\left[\begin{array}{lll}
2 & 1 & 1 \\
2 & 1 & 1
\end{array}\right]-\frac{9}{10} \mathcal{C}\left[\begin{array}{ll}
4 & 0 \\
4 & 0
\end{array}\right]\right) \\
& =-18 \beta^{\mathrm{sv}}\left[\begin{array}{ll}
2 & 0 \\
4 & 4
\end{array}\right]+12 \zeta_{3} \beta^{\mathrm{sv}}\left[\begin{array}{l}
0 \\
4
\end{array}\right]+\frac{5 \zeta_{5}}{12 y}-\frac{\zeta_{3}^{2}}{4 y^{2}} \\
\pi \nabla E_{2,2} & =\frac{(\operatorname{Im} \tau)^{5}}{\pi^{3}}\left(\mathcal{C}\left[\begin{array}{lll}
3 & 1 & 1 \\
1 & 1 & 1
\end{array}\right]-\frac{8}{5} \mathcal{C}\left[\begin{array}{ll}
5 & 0 \\
3 & 0
\end{array}\right]\right) \\
& =9 \beta^{\mathrm{sv}}\left[\begin{array}{ll}
2 & 1 \\
4 & 4
\end{array}\right]-6 \zeta_{3} \beta^{\mathrm{sv}}\left[\begin{array}{l}
1 \\
4
\end{array}\right]-\frac{5 \zeta_{5}}{12}+\frac{\zeta_{3}^{2}}{2 y}
\end{aligned}
$$

of MGFs (2.26) are engineered to avoid $G_{8}$ in the differential equations [51]. The systematics of depth-one relations between eMZVs and non-holomorphic Eisenstein series including higher- 
weight generalizations of (5.13) is detailed in appendix C.1 and leads to the closed-form results

$$
\begin{aligned}
& \mathrm{SV} \sum_{j=0}^{\ell-1} \frac{B_{j}}{j !} \omega\left(0^{\ell-j}, 2 k+\ell \mid-\frac{1}{\tau}\right) \\
& \quad=(-1)^{\ell} \frac{(k+\ell-1) !}{(2 k+\ell-1) !}(-4 \pi \nabla)^{k} E_{k+\ell}, \quad k \geqslant 0, \ell \geqslant 1, k+\ell \geqslant 2 \\
& \mathrm{SV} \sum_{j=0}^{\ell-1} \frac{B_{j}}{j !} \omega\left(0^{\ell-j}, \ell-2 k \mid-\frac{1}{\tau}\right) \\
& \quad=(-1)^{\ell+k} \frac{(\ell-k-1) !}{(\ell-1) !} \frac{(\pi \bar{\nabla})^{k} E_{\ell-k}}{(2 y)^{2 k}}, \quad k \geqslant 0, \ell-2 k \geqslant 1, \ell-k \geqslant 2
\end{aligned}
$$

for combinations of eMZVs of different length that are weighted by Bernoulli numbers $B_{j}$ [104]. Similarly, the analogue of (5.14) for the MGF $E_{2,3}=\left(\frac{\operatorname{Im} \tau}{\pi}\right)^{5}$ $\left(\mathcal{C}\left[\begin{array}{lll}3 & 1 & 1 \\ 3 & 1 & 1\end{array}\right]-\frac{43}{35} \mathcal{C}\left[\begin{array}{ll}5 & 0 \\ 5 & 0\end{array}\right]\right)$ and its holomorphic derivatives is spelled out in appendix C.2.

Note that the single-valued map of A-cycle eMZVs at argument $\tau$ rather than $-\frac{1}{\tau}$ generically leads to combinations of MGFs of different modular weights. For instance, changing the $\operatorname{argument}-\frac{1}{\tau}$ to $\tau$ in $(5.12)$ gives rise to

$$
\operatorname{SV} \omega(0,0,2 \mid \tau)=-\frac{y^{2}}{15}+E_{2}(\tau)+\frac{\pi \bar{\nabla} E_{2}(\tau)}{y}
$$

instead of a single modular invariant $E_{2}(\tau)$. This can be seen by expressing all of $\omega(0,0,2 \mid \tau)$, $E_{2}(\tau)$ and $\bar{\nabla} E_{2}(\tau)$ in terms of convergent iterated Eisenstein integrals (4.34) and applying their single-valued map (4.39). ${ }^{22}$ Alternatively, (5.17) can be deduced by setting $\tau \rightarrow-\frac{1}{\tau}$ in (5.11) and exploiting the result $\mathrm{SV} \omega\left(0,1,0,0 \mid-\frac{1}{\tau}\right)=\frac{3 \pi \bar{\nabla} E_{2}(\tau)}{8 y^{2}}$ that will be extracted from a fourpoint example in section 5.5. The much cleaner result (5.12) for $\operatorname{SV} \omega\left(0,0,2 \mid-\frac{1}{\tau}\right)$ as compared to $\mathrm{SV} \omega(0,0,2 \mid \tau)$ is another manifestation of the fact that the differential equation (3.29) of Bcycle integrals are more closely related to the closed-string counterparts (3.30) than the A-cycle differential equations in (2.19).

\subsection{Symmetrized cycles and graph functions}

At $n \geqslant 3$ points, most of the antielliptic functions in (3.9) introduce non-constant $\overline{f_{i j}^{(a)}}$ into the closed-string integrands, except for the simplest case $V_{0}(1,2, \ldots, n \mid \tau)=1$ dual to a

${ }^{22}$ The representations in terms of convergent iterated Eisenstein integrals needed to verify (5.17) are

$$
\begin{aligned}
& \omega(0,0,2 \mid \tau)=-6 \mathcal{E}_{0}(4,0 ; \tau)-\frac{1}{3} \zeta_{2} \\
& E_{2}(\tau) \quad=\frac{y^{2}}{45}+\frac{\zeta_{3}}{y}-12 \operatorname{Re}\left[\mathcal{E}_{0}(4,0 ; \tau)\right]-\frac{6}{y} \operatorname{Re}\left[\mathcal{E}_{0}(4,0,0 ; \tau)\right] \\
& \pi \bar{\nabla} E_{2}(\tau)=\frac{2 y^{3}}{45}-\zeta_{3}+24 y^{2} \overline{\mathcal{E}_{0}(4 ; \tau)}+12 y \overline{\mathcal{E}_{0}(4,0 ; \tau)}+6 \operatorname{Re}\left[\mathcal{E}_{0}(4,0,0 ; \tau)\right]
\end{aligned}
$$


permutation sum over B-cycles, see (3.38),

$$
\begin{aligned}
J_{0, \eta^{\tau}}^{\tau}(* \mid \rho)= & \int_{\mathfrak{T}^{n-1}}\left(\prod_{j=2}^{n} \frac{\mathrm{d}^{2} z_{j}}{\operatorname{Im} \tau}\right) \prod_{1 \leqslant i<j}^{n} \mathrm{e}^{s_{i j} \mathcal{G}_{\mathfrak{T}}\left(z_{i j}, \tau\right)} \\
& \times \rho\left\{\prod_{k=2}^{n} \sum_{a_{k}=0}^{\infty}(\tau-\bar{\tau})^{a_{k}}\left(\eta_{k, k+1 \ldots n}\right)^{a_{k}-1} f_{k-1, k}^{\left(a_{k}\right)}\right\} \\
= & \mathrm{SV} \sum_{\gamma \in S_{n-1}} B_{\tilde{\eta}^{\tau}}^{\tau}(\gamma \mid \rho) .
\end{aligned}
$$

As indicated by the $*$-notation, the $J_{0, \vec{\eta}}^{\tau}(* \mid \rho)$ integral (3.36) on the left-hand side is independent of the ordering $*$ since its integrand $V_{0}$ is. The symmetrized open-string integrals on the righthand side were studied in $[16,51,77]$ as the generating series of holomorphic graph functions,

$$
\begin{aligned}
\sum_{\gamma \in S_{n-1}} B_{\bar{\eta}}^{\tau}(\gamma \mid \rho)= & \left(\int_{-\tau / 2}^{\tau / 2} \prod_{j=2}^{n} \frac{\mathrm{d} z_{j}}{\tau}\right) \prod_{1 \leqslant i<j}^{n} \mathrm{e}^{s_{i j} \mathcal{G}_{\mathfrak{B}}\left(z_{i j}, \tau\right)} \\
& \times \rho\left\{\prod_{k=2}^{n} \sum_{a_{k}=0}^{\infty} \tau^{a_{k}}\left(\eta_{k, k+1 \ldots n}\right)^{a_{k}-1} f_{k-1, k}^{\left(a_{k}\right)}\right\},
\end{aligned}
$$

where each puncture is integrated independently over the entire B-cycle. More specifically, the references considered the components $f_{k-1, k}^{\left(a_{k}\right)} \rightarrow f_{k-1, k}^{(0)}=1$ at the most singular order in the $\eta_{j}$,

$$
\begin{aligned}
& M_{n}^{\text {open }}=\left.\sum_{\gamma \in S_{n-1}} B_{\bar{\eta}}^{\tau}(\gamma \mid \rho)\right|_{\eta_{23 \ldots n}^{-1} \eta_{3 \ldots n}^{-1} \ldots \eta_{n}^{-1}}=\left(\prod_{j=2}^{n} \int_{-\tau / 2}^{\tau / 2} \frac{\mathrm{d} z_{j}}{\tau}\right) \prod_{1 \leq i<j}^{n} e^{s_{i j} \mathcal{G}_{\mathfrak{B}}\left(z_{i j}, \tau\right)} \\
& =1+\frac{1}{2} \sum_{1 \leq i<j}^{n} s_{i j}^{2} \mathbf{B}[\bullet]+\frac{1}{6} \sum_{1 \leq i<j}^{n} s_{i j}^{3} \mathbf{B}[\bullet]+\sum_{1 \leq i<j<k}^{n} s_{i j} s_{i k} s_{j k} \mathbf{B}[\boldsymbol{\bullet}]+\mathcal{O}\left(s_{i j}^{4}\right),
\end{aligned}
$$

where the dependence on the permutation $\rho$ drops out, and the integrands at fixed order in $s_{i j}$ are polynomials in B-cycle Green functions. In passing to the second line, each monomial in $\mathcal{G}_{\mathfrak{B}}\left(z_{i j}, \tau\right)$ is mapped to a graph $\Gamma$ that labels the B-cycle graph functions $\mathbf{B}[\Gamma]$, where a factor of $\mathcal{G}_{\mathfrak{B}}\left(z_{i j}, \tau\right)$ is represented by an edge connecting vertices $z_{i}$ and $z_{j}$. One-particle reducible graphs $\Gamma_{1 \mathrm{PR}}$ lead to vanishing $\mathbf{B}\left[\Gamma_{1 \mathrm{PR}}\right]$ since $\int_{-\tau / 2}^{\tau / 2} \mathrm{~d} z \mathcal{G}_{\mathfrak{B}}(z, \tau)=0$, i.e. higher orders of $(5.20)$ stem from all combinations of one-particle irreducible graphs with four and more edges in total. Any $\mathbf{B}[\Gamma]$ is expressible in terms of B-cycle eMZVs [51] since the $\alpha^{\prime}$-expansion of each component integral of the series $B_{\tilde{\eta}}^{\tau}(\gamma \mid \rho)$ is.

Similarly, modular graph functions $\mathbf{D}[\Gamma]$ (as opposed to MGFs) were defined $[10,11]$ by $n$-point torus integrals over monomials in $\mathcal{G}_{\mathfrak{T}}\left(z_{i j}, \tau\right)$, where each torus Green function is again visualized through an edge between vertices $z_{i}$ and $z_{j}$. The $\mathbf{D}[\Gamma]$ associated with dihedral graphs $\Gamma$ are proportional to the lattice sums (2.26) with $a_{j}=b_{j}$, and also more complicated graph topologies can be straightforwardly translated into lattice sums. 
The generating series of $n$-point modular graph functions resides at the most singular order of (5.18) w.r.t. $\eta_{j}$ where the insertions of $f_{i j}^{(a)}$ are absent,

$$
\begin{aligned}
& M_{n}^{\text {closed }}=\left.J_{0, \vec{\eta}^{\prime}}^{\tau}(* \mid \rho)\right|_{\eta_{23 \ldots n}^{-1} \eta_{3 \ldots n}^{-1} \ldots \eta_{n}^{-1}}=\int_{\mathfrak{T}^{n-1}}\left(\prod_{j=2}^{n} \frac{\mathrm{d}^{2} z_{j}}{\operatorname{Im} \tau}\right) \prod_{1 \leq i<j}^{n} e^{s_{i j} \mathcal{G}_{\mathfrak{I}}\left(z_{i j}, \tau\right)}
\end{aligned}
$$

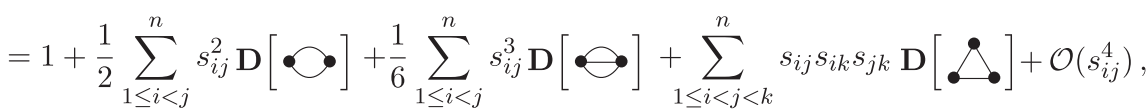

and we have $\mathbf{D}\left[\Gamma_{1 \mathrm{PR}}\right]=0$ by $\int_{\mathfrak{T}} \mathrm{d}^{2} z \mathcal{G}_{\mathfrak{T}}(z, \tau)=0$. As a consequence of (5.18) at the most singular order in the $\eta_{j}$, modular graph functions are single-valued B-cycle graph functions,

$$
M_{n}^{\text {closed }}=\mathrm{SV} M_{n}^{\text {open }} \Leftrightarrow \mathbf{D}[\Gamma]=\mathrm{SV} \mathbf{B}[\Gamma],
$$

which ultimately follows from the 'Betti-deRham duality' between $V_{0}=1$ and the symmetrized cycles $\sum_{\gamma \in S_{n-1}} \mathfrak{B}(\gamma(2, \ldots, n))$.

The relations in (5.22) have firstly appeared in [51] with a proposal 'esv' for an elliptic single-valued map in the place of SV. The esv map of [51] has the same action (3.33) on MZVs and Laurent polynomials in $\tau$ as the SV map in this work. In particular, all pairs of B-cycle eMZVs and modular graph functions related via esv $\omega\left(\ldots \mid-\frac{1}{\tau}\right) \sim \mathbf{D}[\ldots]$ in the reference are also related via $\mathrm{SV} \omega\left(\ldots \mid-\frac{1}{\tau}\right) \sim \mathbf{D}[\ldots]$ as a consequence of (5.22). For suitable representations of the $q$-series of eMZVs via $\mathcal{E}_{0}$ defined by (4.34), the Fourier expansions of all modular graph functions up to weight six could be reproduced from the replacement $\mathcal{E}_{0} \rightarrow 2 \operatorname{Re}\left(\mathcal{E}_{0}\right)$ prescribed by esv [51]. However, it was an open problem in the reference to reconcile esv with the shuffle property of iterated Eisenstein integrals. The SV action (4.30) in turn is expected to be compatible with the shuffle multiplication of the $\beta[\ldots]$ and $\beta^{\mathrm{sv}}[\ldots]$ by the discussion in section 4.3 as detailed below (4.21).

Note that subleading orders in the $\eta_{j}$-expansion of (5.18) generate infinite families of additional relation between MGFs and single-valued eMZVs beyond (5.22). The comparison of open- and closed-string integrals with additional insertions of $f_{12}^{\left(a_{2}\right)} f_{23}^{\left(a_{3}\right)} \ldots f_{n-1, n}^{\left(a_{n}\right)}$ identifies MGFs of various modular weights as single-valued B-cycle eMZVs.

\subsection{Three-point cycles and $\overline{V_{1}(1,2,3 \mid \tau)}$}

The simplest instance of $J_{\vec{\eta}}^{\tau}(\gamma \mid \rho)=\mathrm{SV} B_{\vec{\eta}}^{\tau}(\gamma \mid \rho)$ with non-constant antielliptic integrands $\overline{V(\ldots \mid \tau)}$ occurs at three points. The single-valued map relates an antisymmetric integration cycle on the open-string side in (3.46) to the closed-string integral

$$
\begin{aligned}
J_{1, \eta_{2}, \eta_{3}}^{\tau}(2,3 \mid 2,3)= & -\frac{1}{2 \pi i} \int_{\mathfrak{T}^{2}} \frac{\mathrm{d}^{2} z_{2} \mathrm{~d}^{2} z_{3}}{(\operatorname{Im} \tau)^{2}} \prod_{1 \leqslant i<j}^{3} \mathrm{e}^{s_{i j}} \mathcal{G}_{\mathfrak{T}}\left(z_{i j}, \tau\right) \\
& \times \sum_{a, b=0}^{\infty}(\tau-\bar{\tau})^{a+b} \eta_{23}^{a-1} \eta_{3}^{b-1} f_{12}^{(a)} f_{23}^{(b)}\left(\overline{f_{12}^{(1)}}+\overline{f_{23}^{(1)}}+\overline{f_{31}^{(1)}}\right) .
\end{aligned}
$$


Since contributions with even $a+b$ integrate to zero, the simplest component integrals involve permutations of $f_{12}^{(1)} \overline{f_{12}^{(1)}}$ or $f_{23}^{(1)} \overline{f_{12}^{(1)}}$ at the orders of $\eta_{3}^{-1}$ or $\eta_{23}^{-1}$,

$$
\begin{aligned}
\left.J_{1, \eta_{2}, \eta_{3}}^{\tau}(2,3 \mid 2,3)\right|_{\eta_{23}^{0} \eta_{3}^{-1}}= & -\frac{\operatorname{Im} \tau}{\pi} \int_{\mathfrak{T}^{2}} \frac{\mathrm{d}^{2} z_{2} \mathrm{~d}^{2} z_{3}}{(\operatorname{Im} \tau)^{2}} \prod_{1 \leqslant i<j}^{3} \mathrm{e}^{s_{i j} \mathcal{G}_{\mathfrak{T}}}\left(z_{i j}, \tau\right) \\
& \times f_{12}^{(1)}\left(\overline{f_{12}^{(1)}}+\overline{f_{23}^{(1)}}+\overline{f_{31}^{(1)}}\right) \\
= & \frac{1}{s_{12}}+\frac{s_{123}^{2}}{2 s_{12}} E_{2}+\frac{s_{123}^{3}}{6 s_{12}}\left(E_{3}+\zeta_{3}\right)+\frac{s_{13} s_{23}}{2}\left(3 E_{3}+\zeta_{3}\right) \\
& +\frac{s_{123}^{4}}{s_{12}}\left(E_{2,2}+\frac{3}{20} E_{4}+\frac{1}{8} E_{2}^{2}\right) \\
& +s_{13} s_{23} s_{123}\left(\frac{9}{2} E_{2,2}+\frac{21}{20} E_{4}\right)+\mathcal{O}\left(s_{i j}^{4}\right)
\end{aligned}
$$

which furnish the simplest examples of kinematic poles $\sim s_{i j}^{-1}$ in a $J_{\vec{\eta}}^{\tau}$-series. The corresponding antisymmetrized B-cycle integral features the same types of kinematic poles in component integrals involving $f_{i j}^{(1)}$, e.g. ${ }^{23}$

$$
\begin{aligned}
&\left.\frac{1}{2}\left[B_{\eta_{2}, \eta_{3}}^{\tau}(2,3 \mid 2,3)-B_{\eta_{2}, \eta_{3}}^{\tau}(3,2 \mid 2,3)\right]\right|_{\eta_{23}^{0} \eta_{3}^{-1}} \\
&=\frac{1}{\tau} \int_{-\frac{\tau}{2}<z_{2}<z_{3}<\frac{\tau}{2}} \mathrm{~d} z_{2} \mathrm{~d} z_{3} f_{12}^{(1)} \prod_{1 \leqslant i<j}^{3} \mathrm{e}^{s_{i j} \mathcal{G}_{\mathfrak{B}}\left(z_{i j}, \tau\right)} \\
&=\frac{1}{s_{12}}+\frac{s_{123}^{2}}{s_{12}}\left(\frac{1}{2} \omega\left(0,0,2 \mid-\frac{1}{\tau}\right)+\frac{5 \zeta_{2}}{12}\right) \\
&+\frac{s_{123}^{3}}{s_{12}}\left(\frac{1}{18} \omega\left(0,0,3,0 \mid-\frac{1}{\tau}\right)-\frac{4}{3} \zeta_{2} \omega\left(0,0,1,0 \mid-\frac{1}{\tau}\right)+\frac{\zeta_{3}}{12}\right) \\
&+s_{13} s_{23}\left(\frac{1}{2} \omega\left(0,0,3,0 \mid-\frac{1}{\tau}\right)+\frac{\zeta_{3}}{4}\right) \\
&+\frac{s_{123}^{4}}{s_{12}}\left(-\omega\left(0,0,0,2,2 \mid-\frac{1}{\tau}\right)-\frac{5}{4} \omega\left(0,0,0,0,4 \mid-\frac{1}{\tau}\right)+\frac{1}{8} \omega\left(0,0,4 \mid-\frac{1}{\tau}\right)\right. \\
&\left.+\frac{5}{8} \omega\left(0,0,2 \mid-\frac{1}{\tau}\right)^{2}+\frac{13}{24} \zeta_{2} \omega\left(0,0,2 \mid-\frac{1}{\tau}\right)-2 \zeta_{2} \omega\left(0,0,0,0,2 \mid-\frac{1}{\tau}\right)+\frac{343 \zeta_{4}}{576}\right) \\
& \quad-s_{13} s_{23} s_{123}\left(\frac{9}{2} \omega\left(0,0,0,2,2 \mid-\frac{1}{\tau}\right)+\frac{21}{4} \omega\left(0,0,0,0,4 \mid-\frac{1}{\tau}\right)-\frac{1}{2} \omega\left(0,0,4 \mid-\frac{1}{\tau}\right)\right. \\
&\left.+\frac{9}{4} \omega\left(0,0,2 \mid-\frac{1}{\tau}\right)^{2}+\frac{1}{2} \zeta_{2} \omega\left(0,0,2 \mid-\frac{1}{\tau}\right)-3 \zeta_{2} \omega\left(0,0,0,0,2 \mid-\frac{1}{\tau}\right)+\frac{11 \zeta_{4}}{40}\right) \\
&+\mathcal{O}\left(s_{i j}^{4}\right) .
\end{aligned}
$$

\footnotetext{
${ }^{23} \mathrm{By}$ slight abuse of notation, we denote the ordering of punctures $z_{i}, z_{j}$ on the imaginary axis by $-\frac{\tau}{2}<z_{i}<z_{j}<\frac{\tau}{2}$.
} 
We have used that, by the antisymmetry $f_{12}^{(1)}=-f_{21}^{(1)}$ of the integrand, the contribution from the ordering $-\frac{\tau}{2}<z_{3}<z_{2}<\frac{\tau}{2}$ is minus that of the ordering $-\frac{\tau}{2}<z_{2}<z_{3}<\frac{\tau}{2}$. Comparison of (5.25) with (5.24) confirms the relation (3.46) under the SV map at the respective orders in $s_{i j}$ and $\eta_{j}$. Up to the restriction of the Koba-Nielsen factor to three instead of five punctures, (5.24) and (5.25) are the type of integrals over $f_{i j}^{(1)} \overline{f_{p q}^{(1)}}$ seen in genus-one five-point amplitudes of type II superstrings $[105,106]$.

\subsection{Four-point cycles and $\overline{V_{2}(1,2,3,4 \mid \tau)}$}

The esv map [51] has also been applied to the four-gluon amplitude of the heterotic string [52], where the torus integral ${ }^{24}$

$$
\begin{aligned}
J_{\text {het }}^{\tau} & =\frac{1}{(2 \pi i)^{2}} \int_{\mathfrak{T}^{3}}\left(\prod_{j=2}^{4} \frac{\mathrm{d}^{2} z_{j}}{\operatorname{Im} \tau}\right) \overline{V_{2}(1,2,3,4 \mid \tau)} \prod_{1 \leqslant i<j}^{4} \mathrm{e}^{s_{i j} \mathcal{G} \mathfrak{T}\left(z_{i j}, \tau\right)} \\
& =\left.J_{2, \eta_{2}, \eta_{3}, \eta_{4}}^{\tau}(2,3,4 \mid 2,3,4)\right|_{\eta_{234}^{-1} \eta_{34}^{-1} \eta_{4}^{-1}}
\end{aligned}
$$

was related to the open-string integration cycle dual to (3.39). More specifically, the MGFs in [52]

$$
\begin{aligned}
\left.J_{\text {het }}^{\tau}\right|_{k_{j}^{2}=0}= & -\frac{3 s_{13} \pi \bar{\nabla} E_{2}}{4 y^{2}}-\left(s_{13}^{2}+2 s_{12} s_{23}\right) \frac{\pi \bar{\nabla} E_{3}}{6 y^{2}}+s_{13}\left(s_{12} s_{23}-s_{13}^{2}\right) \\
& \times\left(\frac{\pi \bar{\nabla} E_{4}}{5 y^{2}}+\frac{3 E_{2} \bar{\nabla} E_{2}}{2 y^{2}}+\frac{3 \bar{\nabla} E_{2,2}}{y^{2}}\right)+\mathcal{O}\left(s_{i j}^{4}\right)
\end{aligned}
$$

were proposed to be the single-valued versions of the eMZVs in the $\alpha^{\prime}$-expansion of

$$
\begin{aligned}
B_{\text {het }}^{\tau} & =\frac{1}{6}\left[2 B_{234}^{\tau}+2 B_{432}^{\tau}-B_{243}^{\tau}-B_{342}^{\tau}-B_{324}^{\tau}-B_{423}^{\tau}\right] \\
B_{i j k}^{\tau} & =\left.B_{\eta_{2}, \eta_{3}, \eta_{4}}^{\tau}(i, j, k \mid 2,3,4)\right|_{\eta_{234}^{-1} \eta_{34}^{-1} \eta_{4}^{-1}}
\end{aligned}
$$

namely [4]

$$
\begin{aligned}
\left.B_{\text {het }}^{\tau}\right|_{k_{j}^{2}=0}= & -2 s_{13} \omega\left(0,1,0,0 \mid-\frac{1}{\tau}\right)-\frac{2}{3}\left(s_{13}^{2}+2 s_{12} s_{23}\right) \\
& \times\left[\omega\left(0,1,0,1,0 \mid-\frac{1}{\tau}\right)+\omega\left(0,1,1,0,0 \mid-\frac{1}{\tau}\right)\right]+\frac{4}{3} s_{13}\left(s_{13}^{2}-s_{12} s_{23}\right) \\
& \times\left[\omega\left(0,0,1,0,0,2 \mid-\frac{1}{\tau}\right)+\omega\left(0,0,0,1,0,2 \mid-\frac{1}{\tau}\right)\right. \\
& \left.-\omega\left(0,1,0,1,1,0 \mid-\frac{1}{\tau}\right)-\zeta_{2} \omega\left(0,1,0,0 \mid-\frac{1}{\tau}\right)\right]+\mathcal{O}\left(s_{i j}^{4}\right) .
\end{aligned}
$$

As indicated by $\left.\right|_{k_{j}^{2}=0}$, the $\alpha^{\prime}$-expansions (5.27) and (5.29) have been obtained in the limit of four-point on-shell kinematics with two independent Mandelstam invariants instead of six.

\footnotetext{
${ }^{24}$ The quantity $J_{\text {het }}^{\tau}$ in $(5.26)$ is defined to be $(2 \pi i)^{-2}$ times the complex conjugate of the integral $\mathcal{I}_{1234}^{(2,0)}$ in $(2.44)$ and (4.35) of [52]. Similarly, $B_{\text {het }}^{\tau}$ in (5.28) is obtained from the integral $Z_{1234}^{(2)}$ in section 5.2 of [52] through modular $S$ transformation.
} 
However, the relation (3.35) between $n$-point closed-string and single-valued open-string integrals is conjectured to be valid for the $\frac{1}{2} n(n-1)$ independent Mandelstam variables $\left\{s_{i j}, 1 \leqslant\right.$ $i<j \leqslant n\}$ with $s_{i j}=s_{j i}$. At four points, the corollary

$$
\begin{aligned}
J_{2, \eta_{2}, \eta_{3}, \eta_{4}}^{\tau}(2,3,4 \mid \rho)= & \frac{1}{6} \operatorname{SV}\left[2 B_{\eta_{2}, \eta_{3}, \eta_{4}}^{\tau}(2,3,4 \mid \rho)+2 B_{\eta_{2}, \eta_{3}, \eta_{4}}^{\tau}(4,3,2 \mid \rho)\right. \\
& -B_{\eta_{2}, \eta_{3}, \eta_{4}}^{\tau}(2,4,3 \mid \rho)-B_{\eta_{2}, \eta_{3}, \eta_{4}}^{\tau}(3,4,2 \mid \rho) \\
& \left.-B_{\eta_{2}, \eta_{3}, \eta_{4}}^{\tau}(3,2,4 \mid \rho)-B_{\eta_{2}, \eta_{3}, \eta_{4}}^{\tau}(4,2,3 \mid \rho)\right]
\end{aligned}
$$

of the relation (3.41) between $V_{2}(1,2,3,4 \mid \tau)$ and permutations of the $V(1,2,3,4 \mid \tau)$ functions is claimed to hold for all of $\left\{s_{12}, s_{13}, s_{23}, s_{14}, s_{24}, s_{34}\right\}$ independent. The coefficient of $\eta_{234}^{-1} \eta_{34}^{-1} \eta_{4}^{-1}$ in (5.30) with $\rho=2,3,4$ then implies

$$
J_{\text {het }}^{\tau}=\mathrm{SV} B_{\text {het }}^{\tau}
$$

and explains the relations between the $\alpha^{\prime}$-expansions (5.27) and (5.29) observed in [52] in the on-shell limit $k_{j}^{2}=0$. In particular, the prescription (4.30) for the single-valued map of the iterated-Eisenstein-integral representation of $B_{\text {het }}^{\tau}$ produces the complete $q, \bar{q}$-expansion of the MGFs in (5.27), whereas certain antiholomorphic contributions could not be reproduced by esv in [52].

By applying (5.31) at the level of the $\alpha^{\prime}$-expansions (5.27) and (5.29), one can infer

$$
\begin{aligned}
\frac{\pi \bar{\nabla} E_{2}}{y^{2}}= & \frac{8}{3} \operatorname{SV} \omega\left(0,1,0,0 \mid-\frac{1}{\tau}\right) \\
\frac{\pi \bar{\nabla} E_{3}}{y^{2}}= & 4 \mathrm{SV}\left[\omega\left(0,1,0,1,0 \mid-\frac{1}{\tau}\right)+\omega\left(0,1,1,0,0 \mid-\frac{1}{\tau}\right)\right] \\
\frac{\pi \bar{\nabla} E_{2,2}}{y^{2}}= & \operatorname{SV}\left[\frac{8}{5} \omega\left(0,0,0,0,0,3 \mid-\frac{1}{\tau}\right)+\frac{2}{5} \omega\left(0,0,0,3 \mid-\frac{1}{\tau}\right)\right. \\
& \left.-\frac{11}{75} \omega\left(0,3 \mid-\frac{1}{\tau}\right)-8 \omega\left(0,0,0,0,1,2 \mid-\frac{1}{\tau}\right)+\frac{\zeta_{3}}{3}\right] .
\end{aligned}
$$

Moreover, higher orders in the $\eta_{j}$-expansion of (5.30) yield infinite families of relations between the $\alpha^{\prime}$-expansions of open- and closed-string integrals over additional factors $f_{1 i}^{(a)} f_{i j}^{(b)} f_{j k}^{(c)}$

\subsection{Imaginary cusp forms and double zetas}

We shall finally exemplify the appearance of cuspidal MGFs from single-valued openstring integrals whose Laurent polynomial at the order of $q^{0} \bar{q}^{0}$ vanishes. A systematic study of imaginary cusp forms among the two-loop MGFs can be found in [54], also see [62] for examples of real cusp forms. The simplest imaginary cusp forms occur among the lattice sums $(2.26)$ at modular weights $(5,5)$ whose basis can be chosen ${ }^{25}$ to

\footnotetext{
25 The choice of basis in [18] is tailored to delay the appearance of holomorphic Eisenstein to higher Cauchy-Riemann derivatives as far as possible. That is why the real MGFs $-\frac{21}{4} E_{2,3}-\frac{1}{2} \zeta_{3} E_{2}$ have been added to the imaginary cusp forms $B_{2,3}+\frac{1}{2}\left(\frac{\operatorname{Im} \tau}{\pi}\right)^{5}\left(\mathcal{C}\left[\begin{array}{lll}0 & 2 & 3 \\ 3 & 0 & 2\end{array}\right]-\overline{\mathcal{C}\left[\begin{array}{lll}0 & 2 & 3 \\ 3 & 0 & 2\end{array}\right]}\right)$ in (5.33).
} 
include [18]

$$
\begin{aligned}
& B_{2,3}=\left(\frac{\operatorname{Im} \tau}{\pi}\right)^{5}\left(\mathcal{C}\left[\begin{array}{llll}
0 & 1 & 2 & 2 \\
1 & 1 & 0 & 3
\end{array}\right]-\overline{\mathcal{C}\left[\begin{array}{llll}
0 & 1 & 2 & 2 \\
1 & 1 & 0 & 3
\end{array}\right]}\right)+\frac{\left(\nabla E_{2}\right) \bar{\nabla} E_{3}-\left(\bar{\nabla} E_{2}\right) \nabla E_{3}}{6(\operatorname{Im} \tau)^{2}} \\
& B_{2,3}^{\prime}=B_{2,3}+\frac{1}{2}\left(\frac{\operatorname{Im} \tau}{\pi}\right)^{5}\left(\mathcal{C}\left[\begin{array}{lll}
0 & 2 & 3 \\
3 & 0 & 2
\end{array}\right]-\overline{\mathcal{C}\left[\begin{array}{lll}
0 & 2 & 3 \\
3 & 0 & 2
\end{array}\right]}\right)-\frac{21}{4} E_{2,3}-\frac{1}{2} \zeta_{3} E_{2}
\end{aligned}
$$

The $\beta^{\mathrm{sv}}$-representations involve double-integrals over $G_{4} G_{6}[18]$,

$$
\begin{aligned}
B_{2,3}= & 450 \beta^{\mathrm{sv}}\left[\begin{array}{ll}
2 & 1 \\
4 & 6
\end{array}\right]-450 \beta^{\mathrm{sv}}\left[\begin{array}{ll}
3 & 0 \\
6 & 4
\end{array}\right]+270 \beta^{\mathrm{sv}}\left[\begin{array}{ll}
2 & 1 \\
6 & 4
\end{array}\right]-270 \beta^{\mathrm{sv}}\left[\begin{array}{ll}
1 & 2 \\
4 & 6
\end{array}\right] \\
& -3 \zeta_{3} \beta^{\mathrm{sv}}\left[\begin{array}{l}
1 \\
4
\end{array}\right]-300 \zeta_{3} \beta^{\mathrm{sv}}\left[\begin{array}{l}
1 \\
6
\end{array}\right]+\frac{45 \zeta_{3} \beta^{\mathrm{sv}}\left[\begin{array}{l}
2 \\
6
\end{array}\right]}{y}+\frac{45 \zeta_{5} \beta^{\mathrm{sv}}\left[\begin{array}{l}
0 \\
4
\end{array}\right]}{y} \\
& -\frac{27 \zeta_{5} \beta^{\mathrm{sv}}\left[\begin{array}{l}
1 \\
4
\end{array}\right]}{4 y^{2}}-\frac{13 \zeta_{5}}{120}, \\
B_{2,3}^{\prime}= & 1260 \beta^{\mathrm{sv}}\left[\begin{array}{ll}
2 & 1 \\
4 & 6
\end{array}\right]-840 \zeta_{3} \beta^{\mathrm{sv}}\left[\begin{array}{l}
1 \\
6
\end{array}\right]+\frac{7 \zeta_{5}}{240}-\frac{\zeta_{3}^{2}}{2 y}-\frac{147 \zeta_{7}}{64 y^{2}}+\frac{21 \zeta_{3} \zeta_{5}}{8 y^{3}},
\end{aligned}
$$

and the associated integration constants $\overline{\alpha[\ldots]}$ can be found in the reference and in an ancillary file within the arXiv submission of this article. Both $B_{2,3}, B_{2,3}^{\prime}$ and their Cauchy-Riemann derivatives drop out from $J_{\eta_{2}}^{\tau}$ and $Y_{\eta_{2}}^{\tau}$ at two points. At three points, one can identify their derivatives as single-valued eMZVs,

$$
\begin{aligned}
\pi \nabla B_{2,3}= & \mathrm{SV}\left[-\frac{1}{2} \omega\left(0,0,2,2,2 \mid-\frac{1}{\tau}\right)-2 \omega\left(0,0,0,1,5 \mid-\frac{1}{\tau}\right)-\frac{3}{8} \omega\left(0,0,0,2,4 \mid-\frac{1}{\tau}\right)\right. \\
& +\frac{\zeta_{3}}{8} \omega\left(0,3 \mid-\frac{1}{\tau}\right)+\frac{17}{48} \omega\left(0,3 \mid-\frac{1}{\tau}\right)^{2}+\frac{7}{8} \omega\left(0,0,4 \mid-\frac{1}{\tau}\right) \omega\left(0,0,2 \mid-\frac{1}{\tau}\right) \\
& -\frac{7}{2} \omega\left(0,0,0,3 \mid-\frac{1}{\tau}\right) \omega\left(0,3 \mid-\frac{1}{\tau}\right)+\frac{137}{16} \omega\left(0,0,0,0,6 \mid-\frac{1}{\tau}\right) \\
& \left.-\frac{15}{32} \omega\left(0,0,6 \mid-\frac{1}{\tau}\right)\right] \\
\pi \nabla B_{2,3}^{\prime}= & \mathrm{SV}\left[-\frac{1}{2} \omega\left(0,0,2,2,2 \mid-\frac{1}{\tau}\right)-11 \omega\left(0,0,0,1,5 \mid-\frac{1}{\tau}\right)\right. \\
& +\frac{295}{8} \omega\left(0,0,0,0,6 \mid-\frac{1}{\tau}\right)-\frac{25}{16} \omega\left(0,0,6 \mid-\frac{1}{\tau}\right)-11 \omega\left(0,0,0,3 \mid-\frac{1}{\tau}\right) \\
& \left.\times \omega\left(0,3 \mid-\frac{1}{\tau}\right)+\frac{11}{12} \omega\left(0,3 \mid-\frac{1}{\tau}\right)^{2}-\frac{1}{4} \omega\left(0,0,4 \mid-\frac{1}{\tau}\right) \omega\left(0,0,2 \mid-\frac{1}{\tau}\right)\right] \\
& +\frac{603}{40} \omega\left(0,0,0,7 \mid-\frac{1}{\tau}\right)-\frac{699}{320} \omega\left(0,7 \mid-\frac{1}{\tau}\right) \\
& \left.-\frac{1323}{160} \omega\left(0,3 \mid-\frac{1}{\tau}\right) \omega\left(0,0,4 \mid-\frac{1}{\tau}\right)\right]
\end{aligned}
$$




$$
\begin{aligned}
(\pi \nabla)^{3} B_{2,3}^{\prime}= & \operatorname{SV}\left[-\frac{63}{40} \omega\left(0,3,5 \mid-\frac{1}{\tau}\right)+\frac{567}{64} \omega\left(0,0,8 \mid-\frac{1}{\tau}\right)\right. \\
& \left.-\frac{63}{8} \omega\left(0,3 \mid-\frac{1}{\tau}\right) \omega\left(0,5 \mid-\frac{1}{\tau}\right)\right]
\end{aligned}
$$

by inspecting the contributions of $f_{12}^{(3)} f_{23}^{(3)}$ or $f_{12}^{(4)}$ to $J_{0, \eta_{2}, \eta_{3}}^{\tau}$ and $f_{12}^{(3)}$ to $J_{1, \eta_{2}, \eta_{3}}^{\tau}$. The appearance of the undifferentiated $B_{2,3}$ and $B_{2,3}^{\prime}$ is relegated to the $J_{\vec{\eta}}^{\tau}$-series at four points (or the $Y_{\vec{\eta}}^{\tau}$-series at three points [18]), and comparison with the B-cycle integrals yields

$$
\begin{aligned}
B_{2,3}= & \operatorname{SV}\left[\frac{143}{20} \omega\left(0,0,0,0,0,5 \mid-\frac{1}{\tau}\right)-\frac{11}{2} \omega\left(0,0,0,0,1,4 \mid-\frac{1}{\tau}\right)+2 \omega\left(0,0,0,0,2,3 \mid-\frac{1}{\tau}\right)\right. \\
& -2 \omega\left(0,0,0,1,2,2 \mid-\frac{1}{\tau}\right)-\frac{91}{40} \omega\left(0,0,0,5 \mid-\frac{1}{\tau}\right)-\frac{449}{7200} \omega\left(0,5 \mid-\frac{1}{\tau}\right) \\
& -\frac{5}{12} \omega\left(0,3 \mid-\frac{1}{\tau}\right) \omega\left(0,0,2 \mid-\frac{1}{\tau}\right)+\frac{5}{2} \omega\left(0,0,2 \mid-\frac{1}{\tau}\right) \omega\left(0,0,0,3 \mid-\frac{1}{\tau}\right) \\
& +\frac{23}{12} \omega\left(0,0,2,3 \mid-\frac{1}{\tau}\right)-\frac{15}{2} \omega\left(0,3 \mid-\frac{1}{\tau}\right) \omega\left(0,0,0,0,2 \mid-\frac{1}{\tau}\right) \\
& \left.+\frac{\zeta_{3}}{4} \omega\left(0,0,2 \mid-\frac{1}{\tau}\right)-\frac{43 \zeta_{5}}{480}\right] \\
B_{2,3}^{\prime}= & S V\left[\frac{463}{10} \omega\left(0,0,0,0,0,5 \mid-\frac{1}{\tau}\right)-22 \omega\left(0,0,0,0,1,4 \mid-\frac{1}{\tau}\right)+5 \omega\left(0,0,0,0,2,3 \mid-\frac{1}{\tau}\right)\right. \\
& -2 \omega\left(0,0,0,1,2,2 \mid-\frac{1}{\tau}\right)-\frac{121}{20} \omega\left(0,0,0,5 \mid-\frac{1}{\tau}\right)-\frac{1069}{3600} \omega\left(0,5 \mid-\frac{1}{\tau}\right) \\
& -\frac{1}{6} \omega\left(0,3 \mid-\frac{1}{\tau}\right) \omega\left(0,0,2 \mid-\frac{1}{\tau}\right)+\omega\left(0,0,2 \mid-\frac{1}{\tau}\right) \omega\left(0,0,0,3 \mid-\frac{1}{\tau}\right) \\
& \left.+\frac{25}{6} \omega\left(0,0,2,3 \mid-\frac{1}{\tau}\right)-24 \omega\left(0,3 \mid-\frac{1}{\tau}\right) \omega\left(0,0,0,0,2 \mid-\frac{1}{\tau}\right)-\frac{11 \zeta_{5}}{96}\right] .
\end{aligned}
$$

The open-string counterparts of $B_{2,3}, B_{2,3}^{\prime}$ and their Cauchy-Riemann derivatives involve the simplest combinations of B-cycle eMZVs with an irreducible $\zeta_{3,5}$ in their Laurent polynomials: the methods of [3] (also see appendix B of [51]) yield the following examples of $\tau \rightarrow i \infty$ degenerations in (5.35) and (5.36),

$$
\begin{aligned}
\left.B_{2,3}\right|_{\mathrm{LP}}= & \mathrm{SV}\left[\frac{i T^{3} \zeta_{2}}{5040}+\frac{29 i T \zeta_{4}}{630}+\frac{\zeta_{3} \zeta_{2}}{8}-\frac{8153 i \zeta_{6}}{2880 T}-\frac{9 \zeta_{5} \zeta_{2}}{8 T^{2}}-\frac{73 \zeta_{3} \zeta_{4}}{16 T^{2}}+\frac{1837 i \zeta_{8}}{240 T^{3}}\right. \\
& \left.+\frac{3 i}{10 T^{3}}\left(2 \zeta_{3,5}+5 \zeta_{3} \zeta_{5}\right)-\frac{39 \zeta_{5} \zeta_{4}}{8 T^{4}}+\frac{45 \zeta_{3} \zeta_{6}}{8 T^{4}}+\frac{33 i \zeta_{10}}{20 T^{5}}\right] \\
= & 0 \\
\left.(\pi \nabla)^{3} B_{2,3}^{\prime}\right|_{\mathrm{LP}}= & \operatorname{SV}\left[\frac{T^{8}}{17280}+\frac{i T^{5} \zeta_{3}}{120}+\frac{T^{4} \zeta_{4}}{40}-\frac{7 T^{2} \zeta_{6}}{80}-\frac{8211 \zeta_{8}}{640}\right. \\
& \left.+\frac{63 \zeta_{3,5}}{40}+\frac{189 i \zeta_{5} \zeta_{4}}{8 T}+\frac{63 i \zeta_{3} \zeta_{6}}{4 T}+\frac{14553 \zeta_{10}}{160 T^{2}}\right] \\
= & \frac{2 y^{8}}{135}-\frac{8 y^{5} \zeta_{3}}{15}-\frac{63 \zeta_{3} \zeta_{5}}{4} .
\end{aligned}
$$


One can see from the order of $T^{-3}$ or $y^{-3}$ that the cuspidal nature of $B_{2,3}$ hinges on the depthtwo result $\operatorname{sv} \zeta_{3,5}=-10 \zeta_{3} \zeta_{5}$. The non-vanishing Laurent polynomial of $(\pi \nabla)^{3} B_{2,3}^{\prime}$ is due to the real MGFs $-\frac{21}{4} E_{2,3}-\frac{1}{2} \zeta_{3} E_{2}$ in (5.33).

Note that the simplest instances of $\zeta_{3,7}$ and $\zeta_{3,5,3}$ arise in the Laurent polynomials of Bcycle eMZVs with MGFs (2.26) of weights $\sum_{j=1}^{r}\left(a_{j}+b_{j}\right)=12$ and 14 in their SV mage. The appearance of $\zeta_{3,5,3}$ in modular graph functions and eMZVs can be found in [12, 51], respectively. While $\zeta_{3,7}$ drops out from MGFs under the single-valued map, it enters for instance the $T^{0}$-order of the Laurent polynomial of $\omega\left(0,3,7 \mid-\frac{1}{\tau}\right)$ whose SV image contributes to the quantity $(\pi \nabla)^{4} B_{2,4}^{\prime}$ in section 9.2 of [62]

$$
\begin{aligned}
\left.\omega\left(0,3,7 \mid-\frac{1}{\tau}\right)\right|_{L P}= & -\frac{T^{10}}{1261260}+\frac{2 i T^{5} \zeta_{5}}{315}+\frac{2 T^{4} \zeta_{6}}{63}+\frac{7 i T^{3} \zeta_{7}}{45}+\frac{7 T^{2} \zeta_{8}}{6} \\
& -\zeta_{3,7}-14 \zeta_{3} \zeta_{7}-6 \zeta_{5}^{2}+\frac{27 \zeta_{10}}{2}+\frac{84 i \zeta_{11}}{T}+\frac{30 i \zeta_{5} \zeta_{6}}{T} \\
& +\frac{84 i \zeta_{3} \zeta_{8}}{T}+\frac{1353 \zeta_{12}}{2 T^{2}} .
\end{aligned}
$$

One can eventually find all $\mathbb{Q}$-independent $\mathrm{MZVs}^{26}$ in the Laurent polynomials of B-cycle eMZVs. This follows from both the degeneration limits of the elliptic KZB associator [3] and from the fact that any MZV is expressible via $\mathbb{Q}[2 \pi i]$-linear combinations of multiple modular values [107].

Note that the Laurent polynomials of all B-cycle eMZVs with length + weight $\leqslant 16$ obtained from an FORM implementation [96] of the methods of [3, 51] are available for download from [97].

\subsection{Single-valued map of individual eMZVs}

While the above combinations of single-valued eMZVs were tailored to obtaining a single MGF in the bases of $[18,51]$, we shall now give a closed formula for the single-valued map of individual eMZVs. The integrands of convergent A-cycle eMZVs (2.24) with length $r$ and $n_{1}, n_{r} \neq 1$ arise at the $s_{i j}^{0}$-order of the series $Z_{\vec{\eta}}^{\tau}$ at $r+1$ points. After modular $S$ transformation, one can obtain any convergent $\omega\left(n_{1}, \ldots, n_{r} \mid-\frac{1}{\tau}\right)$ by isolating suitable $\eta_{j}$-orders in the $s_{i j} \rightarrow 0$ limit of $\sum_{\rho \in S_{r}} B_{\vec{\eta}}^{\tau}(1,2, \ldots, r+1 \mid 1, \rho(2,3, \ldots, r+1))$, where the permutation sum over the orderings of the integrands (2.13) yields the integrands $f_{21}^{\left(n_{1}\right)} f_{31}^{\left(n_{2}\right)} \ldots f_{r+1,1}^{\left(n_{r}\right)}$ in the definition (2.24) of eMZVs. Hence, our proposal (3.35) implies that SV $\omega\left(n_{1}, \ldots, n_{r} \mid-\frac{1}{\tau}\right)$ occurs at the corresponding orders of $s_{i j}$ and $\eta_{j}$ in the series $J_{\vec{\eta}}^{\tau}$, so their definition (3.13) leads to $\left(n_{1}, n_{r} \neq 1\right.$ and $\left.z_{1}=0\right)$

$$
\begin{aligned}
\operatorname{SV} \omega\left(n_{1}, n_{2}, \ldots, n_{r} \mid-\frac{1}{\tau}\right)= & (\tau-\bar{\tau})^{n_{1}+\cdots+n_{r}} \int_{\mathfrak{T}^{r}}\left(\prod_{j=2}^{r+1} \frac{\mathrm{d}^{2} z_{j}}{\operatorname{Im} \tau}\right) \\
& \times \overline{V(1,2, \ldots, r+1 \mid \tau)} f_{21}^{\left(n_{1}\right)} f_{31}^{\left(n_{2}\right)} \ldots f_{r+1,1}^{\left(n_{r}\right)} .
\end{aligned}
$$

By the techniques in [52], the integral on the right-hand side can be straightforwardly performed in terms of lattice sums over $p=m \tau+n \in \Lambda^{\prime}$ in (2.25): after expressing the

\footnotetext{
${ }^{26}$ See [102] for a computer implementation of $\mathbb{Q}$-relations among MZVs.
} 


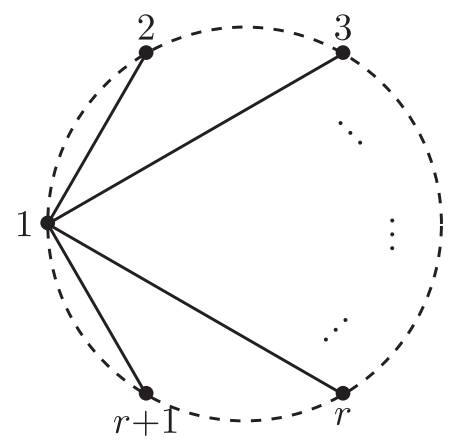

Figure 4. Graphical representation of the integrand of single-valued eMZVs (5.39): solid lines represent factors of $f_{j k}^{(w)}$ while dashed lines stand for the $\overline{f_{12}^{(w)}}, \overline{f_{23}^{(w)}}, \ldots, \overline{f_{r, r+1}^{(w)}}, \overline{f_{r+1,1}^{(w)}}$ that are compatible with the cyclic arrangement of the arguments of $\overline{V(1,2, \ldots, r+1 \mid \tau)}$.

$\overline{V_{w}}$-functions in terms of $\overline{f_{j k}^{(w)}}$ via (5.2) and inserting the double Fourier expansions

$$
f_{j k}^{(w)}=(-1)^{w-1} \sum_{p \in \Lambda^{\prime}} \frac{e^{2 \pi i\left\langle p, z_{j k}\right\rangle}}{p^{w}}, \quad z_{j k}=u_{j k} \tau+v_{j k}, \quad\left\langle p, z_{j k}\right\rangle=m v_{j k}-n u_{j k}
$$

the integrals $\int_{\mathfrak{T}} \frac{\mathrm{d}^{2} z_{j}}{\operatorname{Im} \tau}=\int_{0}^{1} \mathrm{~d} u_{j} \int_{0}^{1} \mathrm{~d} v_{j}$ lead to momentum-conserving delta functions as seen in the dihedral MGFs (2.26). When visualizing each factor of $f_{j k}^{(w)}$ and $\overline{f_{j k}^{(w)}}$ in the integrand of (5.39) through an edge between vertices $j$ and $k$, contributions from one-particle reducible graphs integrate to zero. There are at most $r-1$ factors of $\overline{f_{j k}^{(w)}}$ from the $\overline{V_{w \leqslant r-1}(1,2, \ldots, r+1)}$ in (5.39), and the admissible pairs $(j, k)$ are visualized via dashed lines in figure 4 .

One can anticipate from the example (reproducing (5.12))

$$
\begin{aligned}
\operatorname{SV} \omega\left(0,0,2 \mid-\frac{1}{\tau}\right) & =(\tau-\bar{\tau})^{2} \int_{\mathfrak{T}^{3}}\left(\prod_{j=2}^{4} \frac{\mathrm{d}^{2} z_{j}}{\operatorname{Im} \tau}\right)\left\{\frac{1}{6}-\frac{\overline{V_{1}(1,2,3,4 \mid \tau)}}{4 \pi i}+\frac{\overline{V_{2}(1,2,3,4 \mid \tau)}}{(2 \pi i)^{2}}\right\} f_{41}^{(2)} \\
& =\left(\frac{\operatorname{Im} \tau}{\pi}\right)^{2} \int_{\mathfrak{T}^{3}}\left(\prod_{j=2}^{4} \frac{\mathrm{d}^{2} z_{j}}{\operatorname{Im} \tau}\right) f_{41}^{(2)} f_{41}^{(2)}=\left(\frac{\operatorname{Im} \tau}{\pi}\right)^{2} \sum_{p \in \Lambda^{\prime}} \frac{1}{\mid p^{4}}=E_{2}(\tau)
\end{aligned}
$$

that only small subset of the terms in $\overline{V(1,2, \ldots, r+1 \mid \tau)}$ contribute to generic single-valued eMZVs - the right-hand side of (5.41) entirely stems from $\overline{V_{2}(1,2,3,4 \mid \tau)} \rightarrow \overline{f_{41}^{(2)}}$. Apart from the restriction to one-particle irreducible graphs, only those $\overline{V_{w}(1,2, \ldots, r+1 \mid \tau)}$ with parity $(-1)^{w}=(-1)^{n_{1}+n_{2}+\cdots+n_{r}}$ contribute since lattice sums with odd overall modular weight vanish.

Note that the torus integrals in the expression (5.39) for single-valued eMZVs converge whenever the eMZVs themselves do: the convergence criterion $n_{1}, n_{r} \neq 1$ rules out any double pole $\left|z_{j k}\right|^{-2}$ in the integrand (and kinematic poles $s_{j k}^{-1}$ in the Koba-Nielsen integral) since the only overlap between the solid and dashed lines in figure 4 occurs via $f_{12}^{\left(n_{1}\right)} \overline{f_{12}^{(w)}}$ and $f_{1, r+1}^{\left.f_{n}\right)} \overline{f_{1, r+1}^{(w)}}$.

Based on the conjectural relation (3.23) between the Laurent polynomials, one can use (5.39) to infer the asymptotics of the MGFs on the right-hand side by importing the Laurent polynomials of the B-cycle eMZVs from [97] and applying the single-valued map. Moreover, 
any relation among eMZVs induces a relation among the MGFs through the lattice-sum representation of their single-valued images. Hence, the database of MGF relations [62] can be complemented by applying (5.39) to the eMZV relations on the website [92]. The lattice sums contributing to $\mathrm{SV} \omega\left(n_{1}, \ldots, n_{r} \mid-\frac{1}{\tau}\right)$ have (anti-)holomorphic modular weights $(w, \bar{w})$ subject to $w=n_{1}+\cdots+n_{r}$ and $\bar{w} \leqslant r-1$. Accordingly, the Laurent polynomials of B-cycle eMZVs of length + weight $\leqslant 16$ [97] give access to those of various combinations of MGFs with $w+\bar{w} \leqslant 15$.

In the remainder of this section, we will study the single-valued eMZVs in (5.39) at fixed length $r$ and comment on consistency checks with the properties of their SV preimages [17]. The condition $n_{1}, n_{r} \neq 1$ for convergence is taken to hold throughout. At length $r=1$, for instance, $\omega\left(2 k \mid-\frac{1}{\tau}\right)=-2 \zeta_{2 k}$ and $\omega\left(2 k-1 \mid-\frac{1}{\tau}\right)=0$ with $k \geqslant 1$ are annihilated by SV, in lines with the vanishing of the torus integral over a single $f_{12}^{(w)}$ at $w \neq 0$.

5.7.1. Length-two examples $\omega\left(n_{1}, n_{2}\right)$. Single-valued eMZVs of length $r=2$ take the form (5.39)

$$
\begin{aligned}
\mathrm{SV} \omega\left(n_{1}, n_{2} \mid-\frac{1}{\tau}\right)= & (\tau-\bar{\tau})^{n_{1}+n_{2}} \int_{\mathfrak{T}^{2}} \frac{\mathrm{d}^{2} z_{2}}{\operatorname{Im} \tau} \frac{\mathrm{d}^{2} z_{3}}{\operatorname{Im} \tau} f_{21}^{\left(n_{1}\right)} f_{31}^{\left(n_{2}\right)} \\
& \times\left\{\begin{array}{ll}
\frac{1}{2} & : n_{1}+n_{2} \text { even } \\
-\frac{V_{1}(1,2,3 \mid \tau)}{2 \pi i} & : n_{1}+n_{2} \text { odd }
\end{array},\right.
\end{aligned}
$$

where the distinction between even and odd weight $n_{1}+n_{2}$ stems from the vanishing of lattice sums with odd modular weight. At even $n_{1}+n_{2}>0$, the integrand of (5.42) is proportional to $f_{21}^{\left(n_{1}\right)} f_{31}^{\left(n_{2}\right)}$ which corresponds to a one-particle reducible graph with

$$
\left.\mathrm{SV} \omega\left(n_{1}, n_{2} \mid-\frac{1}{\tau}\right)\right|_{n_{1}+n_{2}>0 \text { even }}=0
$$

in agreement with $\omega\left(2 k_{1}, 2 k_{2} \mid-\frac{1}{\tau}\right)=2 \zeta_{2 k_{1}} \zeta_{2 k_{2}}$ and $\omega\left(2 k_{1}-1,2 k_{2}-1 \mid-\frac{1}{\tau}\right)=0$ [17]. For odd weight $n_{1}+n_{2}$, we keep $n_{2} \neq 0$ and distinguish the two cases $n_{1}=0$ and $n_{1} \neq 0$, where the only contributions of $\overline{V_{1}(1,2,3 \mid \tau)}$ to the integral (5.42) stem from $\overline{f_{31}^{(1)}}$ and $\overline{f_{23}^{(1)}}$, respectively:

$$
\begin{aligned}
\left.\operatorname{SV} \omega\left(0, n_{2} \mid-\frac{1}{\tau}\right)\right|_{n_{2} \text { odd }} & =-\frac{(\tau-\bar{\tau})^{n_{2}}}{2 \pi i} \int_{\mathfrak{T}} \frac{\mathrm{d}^{2} z_{3}}{\operatorname{Im} \tau} f_{31}^{\left(n_{2}\right)} \overline{f_{31}^{(1)}}=\frac{i}{2 \pi}(\tau-\bar{\tau})^{n_{2}} \mathcal{C}\left[\begin{array}{cc}
n_{2} & 0 \\
1 & 0
\end{array}\right] \\
\left.\operatorname{SV} \omega\left(n_{1}, n_{2} \mid-\frac{1}{\tau}\right)\right|_{n_{1}+n_{2} \text { odd }} ^{n_{1}, n_{2} \neq 0} & =-\frac{(\tau-\bar{\tau})^{n_{1}+n_{2}}}{2 \pi i} \int_{\mathfrak{T}} \frac{\mathrm{d}^{2} z_{2}}{\operatorname{Im} \tau} \frac{\mathrm{d}^{2} z_{3}}{\operatorname{Im} \tau} f_{21}^{\left(n_{1}\right)} f_{31}^{\left(n_{2}\right)} \overline{f_{23}^{(1)}} \\
& =(-1)^{n_{1}} \frac{i}{2 \pi}(\tau-\bar{\tau})^{n_{1}+n_{2}} \mathcal{C}\left[\begin{array}{cc}
n_{1}+n_{2} & 0 \\
1 & 0
\end{array}\right]
\end{aligned}
$$

The resulting relation $\mathrm{SV} \omega\left(n_{1}, n_{2} \mid-\frac{1}{\tau}\right)=(-1)^{n_{1}} \mathrm{SV} \omega\left(0, n_{1}+n_{2} \mid-\frac{1}{\tau}\right)$ is consistent with (2.33) of [17] after discarding any $\mathrm{SV} \zeta_{2 k}$ with $k \geqslant 1$ from the equation of the reference. In combination with the vanishing of SV $\left.\omega\left(n_{1}, n_{2}\right)\right|_{n_{1}+n_{2}}$ even, we conclude that all single-valued eMZVs of length two do not exceed one-loop MGFs, in lines with $\omega(0,2 k+1)$ being iterated Eisenstein integrals of depth one. 
5.7.2. Length-three examples $\omega\left(n_{1}, n_{2}, n_{3}\right)$. Single-valued eMZVs of length three can be written as

$$
\begin{aligned}
\operatorname{SV} \omega\left(n_{1}, n_{2}, n_{3} \mid-\frac{1}{\tau}\right)= & (\tau-\bar{\tau})^{n_{1}+n_{2}+n_{3}} \int_{\mathfrak{T}^{3}}\left(\prod_{j=2}^{4} \frac{\mathrm{d}^{2} z_{j}}{\operatorname{Im} \tau}\right) f_{21}^{\left(n_{1}\right)} f_{31}^{\left(n_{2}\right)} f_{41}^{\left(n_{3}\right)} \\
& \times \begin{cases}\frac{1}{6}+\frac{\overline{V_{2}(1,2,3,4 \mid \tau)}}{(2 \pi i)^{2}} & : n_{1}+n_{2}+n_{3} \text { even } \\
-\frac{V_{1}(1,2,3,4 \mid \tau)}{4 \pi i} & : n_{1}+n_{2}+n_{3} \text { odd }\end{cases}
\end{aligned}
$$

after discarding lattice sums of odd modular weight. For SV $\omega\left(0,0,2 k+2 \mid-\frac{1}{\tau}\right)$, this results in the one-loop MGFs in the second line of (C.2). Starting from weight 8, the bases of $\omega\left(n_{1}, n_{2}, n_{3}\right)$ require representatives with two non-zero entries such as $\omega(0,3,5), \omega(0,3,7)$ [17], and their single-valued versions correspond to MGFs of depth two as exemplified in section 5.6. For any combination of three non-vanishing entries, the torus integral in (5.45) can be expressed in terms of two-loop MGFs $\left[\begin{array}{lll}a_{1} & a_{2} & a_{3} \\ b_{1} & b_{2} & b_{3}\end{array}\right]$ in (2.26) and products of one-loop MGFs,

$$
\begin{aligned}
& \operatorname{SV} \omega\left(n_{1}, n_{2}, n_{3} \mid-\frac{1}{\tau}\right) \mid \begin{array}{l}
n_{1}, n_{2}, n_{3} \neq 0 \\
n_{1}+n_{2}+n_{3} \text { odd }
\end{array}=0 \\
& \operatorname{SV} \omega\left(n_{1}, n_{2}, n_{3} \mid-\frac{1}{\tau}\right) \mid \begin{array}{l}
n_{1}, n_{2}, n_{3} \neq 0 \\
n_{1}+n_{2}+n_{3}
\end{array} \text { even } \\
& =\frac{(\tau-\bar{\tau})^{n_{1}+n_{2}+n_{3}}}{(2 \pi i)^{2}}\left\{(-1)^{n_{3}} \mathcal{C}\left[\begin{array}{cc}
n_{1} & 0 \\
1 & 0
\end{array}\right] \mathcal{C}\left[\begin{array}{cc}
n_{2}+n_{3} & 0 \\
1 & 0
\end{array}\right]\right. \\
& \left.\quad+(-1)^{n_{1}} \mathcal{C}\left[\begin{array}{cc}
n_{3} & 0 \\
1 & 0
\end{array}\right] \mathcal{C}\left[\begin{array}{cc}
n_{1}+n_{2} & 0 \\
1 & 0
\end{array}\right]+\mathcal{C}\left[\begin{array}{ccc}
n_{1} & n_{2} & n_{3} \\
1 & 0 & 1
\end{array}\right]\right\},
\end{aligned}
$$

leading to iterated Eisenstein integrals of depth two.

5.7.3. Length four and beyond. Starting from single-valued eMZVs of length four

$$
\begin{aligned}
\operatorname{SV} \omega & \left(n_{1}, n_{2}, n_{3}, n_{4} \mid-\frac{1}{\tau}\right) \\
= & (\tau-\bar{\tau})^{n_{1}+n_{2}+n_{3}+n_{4}} \int_{\mathfrak{T}^{4}}\left(\prod_{j=2}^{5} \frac{\mathrm{d}^{2} z_{j}}{\operatorname{Im} \tau}\right) f_{21}^{\left(n_{1}\right)} f_{31}^{\left(n_{2}\right)} f_{41}^{\left(n_{3}\right)} f_{51}^{\left(n_{4}\right)} \\
& \times \begin{cases}\frac{1}{24}+\frac{\overline{V_{2}(1,2,3,4,5 \mid \tau)}}{2(2 \pi i)^{2}} & : n_{1}+n_{2}+n_{3}+n_{4} \text { even } \\
-\frac{V_{1}(1,2,3,4,5 \mid \tau)}{12 \pi i}-\frac{V_{3}(1,2,3,4,5 \mid \tau)}{(2 \pi i)^{3}} & : n_{1}+n_{2}+n_{3}+n_{4} \text { odd }\end{cases}
\end{aligned}
$$

MGFs of different modular weights may mix through the contributions of $\overline{V_{1}}$ and $\overline{V_{3}}$ to

$$
\left.\operatorname{SV} \omega\left(0,0,0, n_{4} \mid-\frac{1}{\tau}\right)\right|_{n_{4} \text { odd }}=-\frac{(\tau-\bar{\tau})^{n}}{12 \pi i} \mathcal{C}\left[\begin{array}{cc}
n_{4} & 0 \\
1 & 0
\end{array}\right]-\frac{(\tau-\bar{\tau})^{n}}{(2 \pi i)^{3}} \mathcal{C}\left[\begin{array}{cc}
n_{4} & 0 \\
3 & 0
\end{array}\right]
$$


The first term drops out when adding $-\left.\frac{1}{6} \mathrm{SV} \omega\left(0, n_{4} \mid-\frac{1}{\tau}\right)\right|_{n_{4} \text { odd }}$ in (5.44) and explains the combinations of eMZVs of different length in the third line of (C.2). Similarly, the more general combinations (5.16) of single-valued eMZVs of different length that isolate one-loop MGFs can be understood from the combinations of $\overline{V_{w}}$ that contribute to higher-point $\bar{V}$-functions (3.8).

With multiple non-zero entries in SV $\omega\left(n_{1}, n_{2}, n_{3}, n_{4} \mid-\frac{1}{\tau}\right)$ of odd weight, the lattice sums from integrating $\overline{V_{3}}$ correspond to MGFs of trihedral topology. Similarly, single-valued eMZVs at length five introduce four-point MGFs of kite topology introduced in section 4.3 of [62]. We hope that their identification with single-valued eMZVs will facilitate the study of MGFs beyond the dihedral topology and result in efficient methods to determine their Laurent polynomials and relations at arbitrary weight.

\section{Conclusions and outlook}

In this work, we have studied generating series of configuration-space integrals that arise in open- and closed-string amplitudes at genus one. The differential equations and $\tau \rightarrow i \infty$ degenerations of these generating series served as a framework to propose the explicit form of an elliptic single-valued map. Our construction is based on a tentative genus-one uplift of the Betti-deRham duality between integration cycles on a disk boundary and antiholomorphic Parke-Taylor integrands which drives the relation between closed-string and single-valued open-string tree amplitudes [40-45]. These considerations lead us to construct closed-string genus-one integrals over specific antielliptic functions which are thought of as Betti-deRham dual to open-string integration cycles in view of their singularities at $z_{i} \rightarrow z_{j}$ and their degeneration at $\tau \rightarrow i \infty$.

Most importantly, the differential equations of the open- and closed-string integrals under investigation only differ by $\tau^{j} G_{k}(\tau)$ vs $(\tau-\bar{\tau})^{j} G_{k}(\tau)$ in the respective differential operators with holomorphic Eisenstein series $G_{k}$. Accordingly, we generate the eMZVs and MGFs in their $\alpha^{\prime}$-expansions via path ordered exponentials with the same polynomial structures in kinematic invariants and formal expansion variables. The $\tau$-dependent building blocks are iterated Eisenstein integrals in both cases - holomorphic ones with kernels $\tau^{j} G_{k}(\tau)$ for the open-string integrals and their single-valued versions involving kernels $(\tau-\bar{\tau})^{j} G_{k}(\tau)$ for closed strings.

Our proposal for an elliptic single-valued map is defined through the relation between the generating series of open- and closed-string integrals. By their respective $\alpha^{\prime}$-expansion, we obtain the single-valued map for all iterated Eisenstein integrals occurring in the open-string series. This in turn determines the single-valued map of any convergent eMZV in terms of MGFs.

This construction hinges on the compatibility of the initial values at $\tau \rightarrow i \infty$ under the single-valued map of MZVs [46, 48]. We have given evidence for their compatibility by identifying the key building blocks of genus-zero integrals at the cusp-appropriate pairs of disk orderings and Parke-Taylor integrands. However, the detailed expressions for the asymptotic expansions beyond two points in terms of genus-zero integrals is left for future work. At present, the procedure also relies on the reality properties of a generating series $Y_{\vec{\eta}}^{\tau}$ of a more general class of closed-string integrals. Our method does not yet provide a direct construction of single-valued iterated Eisenstein integrals solely from open-string data.

\subsection{Genus-one integrals versus string amplitudes}

The results of this work concern infinite families of configuration-space integrals at genus one, and their application to genus-one string amplitudes requires the following leftover steps: 
For both open and closed strings, it remains to integrate over the modular parameter $\tau$ of the respective surface. In the closed-string case, $\tau$-integrals over MGFs are typically performed on the basis of their Laplace equations [9, 108-112] and Poincaré-series representations [54, 113-118]. The $\tau$-integration of open-string integrals has for instance been discussed in [119-122], and a general method applicable to arbitrary depth may be based on the representation of eMZVs in terms of iterated Eisenstein integrals (including their 'over-integrated' instances with kernels $\tau^{j} G_{k}$ at $j>k-2$ [118]) and properties of multiple modular values [101]. It would be particularly interesting to relate closed-string and single-valued open-string integrals at genus one after integration over $\tau$.

For open strings, the $Z_{\vec{\eta}}^{\tau}$ - or $B_{\vec{\eta}^{-}}^{\tau}$-series are claimed to exhaust all the configuration-space integrands built from $f^{(k)}\left(z_{i}-z_{j}, \tau\right)$ that are inequivalent under Fay identities and integration by parts. Similarly, the $Y_{\vec{\eta}}^{\tau}$-series built from double copies of the open-string integrands is expected to contain all torus integrals of this type. Hence, by the arguments of $[4,52,79], B_{\vec{\eta}}^{\tau}$ and $Y_{\vec{\eta}}^{\tau}$ should $^{27}$ capture the conformal-field-theory correlators in the integrands of $n$-point genus-one amplitudes of massless states (and possibly also of massive states) in bosonic, heterotic and type-II string theories. In all cases, the component integrals in the $\eta_{j}$-expansions of the $Z_{\vec{\eta}}^{\tau}$, $B_{\vec{\eta}}^{\tau}$ or $Y_{\vec{\eta}}^{\tau}$ series need to be dressed with kinematic factors that are determined by the correlators and carry the polarization dependence of the respective string amplitude.

The integrands of $J_{\vec{\eta}}^{\tau}$ only involve antielliptic combinations of $\overline{f^{(k)}\left(z_{i}-z_{j}, \tau\right)}$ and omit infinite classes of component integrals of $Y_{\vec{\eta}}^{\tau}$. For a given genus-one closed-string amplitude, it is therefore not a priori clear if its correlator is generated by the integrand of $J_{\vec{\eta}}^{\tau}$. Still, the correlators for the four- and five-point type-II amplitudes can be recovered from the subsectors $J_{w, \vec{\eta}}^{\tau}$ of the $J_{\vec{\eta}}^{\tau}$-series at fixed modular weights: the four-point correlator of [80] resides at the $\eta^{-3}$ order of $J_{0, \eta_{2}, \eta_{3}, \eta_{4}}^{\tau}$, and the five-point correlators of $[105,106]$ can be assembled from the most singular $\eta$-orders of $\boldsymbol{J}_{w, \eta_{2}, \eta_{3}, \eta_{4}, \eta_{5}}$ at $w=0,1$. Similarly, the four- and five-point amplitudes of gluons and gravitons in heterotic string theories can in principle be extracted from the same $J_{w, \vec{\eta}}^{\tau}$ which also appear in type II, where higher orders in $\eta_{j}$ are needed to capture the bosonic sectors. It would be interesting to see if this pattern persists at higher points in supersymmetric amplitudes, and whether the $J_{\vec{\eta}}^{\tau}$ are sufficient to generate bosonic-string amplitudes at low multiplicity.

\subsection{Further directions}

This work spawns a variety of further directions and open questions of relevance to both physicists and mathematicians:

The single-valued image of eMZVs is proposed to contain combinations of holomorphic iterated Eisenstein integrals and their complex conjugates denoted by $\beta^{\text {sv }}$ and constructed from the $\alpha^{\prime}$-expansion of closed-string integrals in [18]. It would be important to work out their detailed relation to Brown's earlier construction of single-valued iterated Eisenstein integrals $[13,14]$. In particular, it remains to relate the MZVs in the antiholomorphic contributions to $\beta^{\text {sv }}$ (fixed from reality properties of Koba-Nielsen integrals in [18]) to the combinations of multiple modular values entering Brown's construction. This will hopefully bypass the need to use these reality properties as independent input for the construction of $\beta^{\text {sv }}$ as done so far.

\footnotetext{
${ }^{27}$ It has been shown in [52] that the integrands of massless genus-one amplitudes in bosonic, heterotic and type-II theories are expressible in terms of products of $f^{(k)}\left(z_{i}-z_{j}, \tau\right)$ and their $z_{i}$-derivatives. The conjectural part is that arbitrary products of $f^{(k)}\left(z_{i}-z_{j}, \tau\right)$ (possibly including derivatives) are expressible in terms of the $\varphi_{\vec{\eta}}^{\tau}$ in (2.13) with their specific chain structure via repeated use of Fay identities and integration by parts $[59,60]$.
} 
Several aspects of our construction are based on conjectures with strong support from a variety of non-trivial examples. As pointed out in the relevant passages in earlier parts of this work, it would be desirable to find mathematically rigorous proofs that

- Any Koba-Nielsen integral at genus one involving products and derivatives of Kronecker-Eisenstein coefficients $f^{(k)}(z, \tau)$ can be expanded in the coefficients of the series $Z_{\vec{\eta}}^{\tau}$ and $Y_{\vec{\eta}}^{\tau}$

- The matrices $r_{\vec{\eta}}\left(\epsilon_{k}\right)$ and $R_{\vec{\eta}}\left(\epsilon_{k}\right)$ in open- and closed-string differential equation (2.19) preserve the commutation relations of Tsunogai's derivations $\epsilon_{k}$

- The single-valued images $\beta^{\text {sv }}$ of iterated Eisenstein integrals satisfy shuffle relations, i.e. that the antiholomorphic integration constants $\alpha$ do not introduce any obstructions

- The coefficients of the $s_{i j^{-}}$and $\eta_{j}$-expansion of the initial values $\widehat{J}_{\vec{\eta}}^{i \infty}$ and $\widehat{Y}_{\vec{\eta}}^{i \infty}$ are singlevalued multiple zeta values

The proposal of the present work concerns single-valued integration [48, 49] in the modular parameter $\tau$. An alternative approach is to recover MGFs from single-valued functions of torus punctures $[10,15]$. In this context, it would be rewarding to find an explicit realization of single-valued integration in $z$ for elliptic polylogarithms and their complex conjugates, for instance by building upon the ideas of [15] and the depth-one results in [123].

At genus zero, the identification of sphere integrals as single-valued disk integrals is equivalent to the Kawai-Lewellen-Tye (KLT) relations between closed-string and squares of openstring tree-level amplitudes [124]. Accordingly, one could wonder if the combinations of holomorphic and antiholomorphic iterated Eisenstein integrals in the $\beta^{\mathrm{sv}}$ or MGFs can arise from products of open-string type generating functions and their complex conjugates. If such a genus-one echo of KLT relations exists, then one can expect a close connection to the monodromy relations among open-string integrals [122, 125-127] and in particular their study in the light of twisted deRham theory [128]. And it could open up a new perspective on the quest for loop-level KLT relations to revisit the generating functions of closed-string integrals in the framework of chiral splitting $[129,130]$, by performing the $\alpha^{\prime}$-expansion at the level of the loop integrand.

A particularly burning question concerns a higher-genus realization of single-valued integration and the associated relations between open- and closed-string amplitudes. A promising first step could be to identify suitable holomorphic open-string analogues of the MGFs [131, 132] and modular graph tensors [133] at higher genus. More generally, the simplified correlators of maximally supersymmetric genus-two amplitudes at four points [134, 135] and five points $[136,137]$ provide valuable showcases of Koba-Nielsen integrals relevant to open- and closed-string scattering. Furthermore, the construction of the generating series in this work was inspired by extended families of genus-one Koba-Nielsen integrals that arise from heterotic or bosonic strings [52]. Hence, the genus-two correlators of the heterotic string and the combinations of theta functions studied in $[138,139]$ could give important clues on higher-genus versions of the elliptic functions and generating series in this work.

\section{Acknowledgments}

We would like to thank Johannes Broedel, Eric D'Hoker, Daniele Dorigoni, Clément Dupont, André Kaderli, Erik Panzer, Oliver Schnetz, Federico Zerbini and in particular Nils Matthes for combinations of valuable discussions and collaboration on related topics. Moreover, we 
are grateful to Johannes Broedel, Eric D'Hoker, André Kaderli and an anonymous referee for valuable comments on a draft. OS thanks AEI Potsdam and AK \& JG thank Uppsala University for hospitality during various stages of this work. JG, CRM and OS are grateful to the organizers of the program 'Modular forms, periods and scattering amplitudes' at the ETH Institute for Theoretical Studies for providing a stimulating atmosphere and financial support during early stages of this project. JG was supported by the International Max Planck Research School for Mathematical and Physical Aspects of Gravitation, Cosmology and Quantum Field Theory during most stages of this work. CRM is supported by a University Research Fellowship from the Royal Society. OS and BV are supported by the European Research Council under ERC-STG-804286 UNISCAMP.

\section{Data availability statement}

All data that support the findings of this study are included within the article (and any supplementary files).

\section{Appendix A. Relations among the elliptic $V$ and $V_{w}$ functions}

In this appendix, we spell out a method to determine the rational coefficients $c_{w, \gamma}$ in the expansion (3.37) of elliptic functions $V_{w}(\ldots)$ of fixed modular weights in terms of the $V(\ldots)$ functions in (3.8). This will be done by exploiting the $\tau \rightarrow i \infty$ degeneration (3.20) of the $V(\ldots)$ which fixes the $c_{w, \gamma}$ in the ansatz (3.37) via

$$
\begin{aligned}
\lim _{\tau \rightarrow i \infty} \frac{V_{w}(1,2, \ldots, n \mid \tau)}{(2 \pi i)^{w} \sigma_{1} \sigma_{2} \ldots \sigma_{n}}= & \sum_{\gamma \in S_{n-1}} c_{w, \gamma} \mathrm{PT}^{(1)}(1, \gamma(2,3, \ldots, n)) \\
\mathrm{PT}^{(1)}(1,2, \ldots, n)= & (-1)^{n-1} \lim _{\sigma_{-} \rightarrow \infty}\left|\sigma_{-}\right|^{2}(\mathrm{PT}(+, n, n-1, \ldots, 2,1,-) \\
& +\operatorname{cyc}(1,2, \ldots, n)) \\
= & \frac{1}{\sigma_{12} \sigma_{23} \ldots \sigma_{n-1, n} \sigma_{n}}+\operatorname{cyc}(1,2, \ldots, n)
\end{aligned}
$$

The combinations $\mathrm{PT}^{(1)}$ are known as one-loop Parke-Taylor factors from an ambitwistorstring context [94], and we have used $\sigma_{+}=0$ in passing to the last line. In order to determine the degeneration of the left-hand side of (A.1), we expand the elliptic functions

$$
\begin{aligned}
& V_{w}(1,2, \ldots, n \mid \tau)=\sum_{\substack{a_{1}, a_{2}, \ldots, a_{n} \geqslant 0 \\
a_{1}+a_{2}+\cdots+a_{n}=w}} g_{12}^{\left(a_{1}\right)} g_{23}^{\left(a_{2}\right)} \ldots g_{n-1, n}^{\left(a_{n-1}\right)} g_{n, 1}^{\left(a_{n}\right)} \\
& \frac{\theta_{1}^{\prime}(0, \tau) \theta_{1}\left(z_{i j}+\eta, \tau\right)}{\theta_{1}\left(z_{i j}, \tau\right) \theta_{1}(\eta, \tau)}=\sum_{a=0}^{\infty} \eta^{a-1} g_{i j}^{(a)}
\end{aligned}
$$


in terms of the meromorphic Kronecker-Eisenstein coefficients $g_{i j}^{(a)}$ instead of the $f_{i j}^{(a)}$ in (5.2), starting with $g_{i j}^{(0)}=1$ and $g_{i j}^{(1)}=\partial_{z_{i}} \log \theta_{1}\left(z_{i j}, \tau\right)$. Their $\tau \rightarrow i \infty$ limits [3, 4]

$$
\lim _{\tau \rightarrow i \infty} g_{j k}^{(a)}= \begin{cases}1 & : a=0 \\ i \pi \frac{\sigma_{j}+\sigma_{k}}{\sigma_{j}-\sigma_{k}} & : a=1 \\ -2 \zeta_{a} & : a \in 2 \mathbb{N} \\ 0 & : a \in 2 \mathbb{N}+1\end{cases}
$$

generated by (3.19) ensure that the combination $(2 \pi i)^{-w} V_{w}(1,2, \ldots, n \mid \tau)$ in (A.1) degenerates to a rational function of the $\sigma_{j}$, where all factors of $i \pi$ cancel. Hence, the only $\sigma_{j}$ dependence of $V_{w}(\ldots \mid \tau \rightarrow i \infty)$ occurs via $\lim _{\tau \rightarrow i \infty} g_{j k}^{(1)}=i \pi \frac{\sigma_{j}+\sigma_{k}}{\sigma_{j}-\sigma_{k}}$.

By applying the degeneration (A.4) to the elliptic function $V_{w}$ in (A.3), the leftover challenge in determining the $c_{w, \gamma}$ in (A.1) is to expand the terms of the form $\left(\sigma_{1} \sigma_{2} \ldots \sigma_{n}\right)^{-1} \prod_{i=1}^{r} \frac{\sigma_{j_{i}}+\sigma_{k_{i}}}{\sigma_{j_{i}}-\sigma_{k_{i}}}$ on the left-hand side in terms of Parke-Taylor factors. For the choices of $\sigma_{j_{i}}, \sigma_{k_{i}}$ that arise from the degeneration of $V_{w \leqslant n-2}$, these Parke-Taylor decompositions can be performed by the methods of [140]: as explained in section 3 of the reference, the net effect of the rational factor $\frac{\sigma_{j_{i}}+\sigma_{k_{i}}}{\sigma_{j_{i}}-\sigma_{k_{i}}}$ is to modify the signs of the Parke-Taylor factors on the right-hand side of

$$
\frac{1}{\sigma_{1} \sigma_{2} \ldots \sigma_{n}}=(-1)^{n-1} \lim _{\sigma_{-} \rightarrow \infty}\left|\sigma_{-}\right|^{2} \sum_{\rho \in S_{n}} \mathrm{PT}(+, \rho(1,2, \ldots, n),-)
$$

More specifically, with the notation

$$
\operatorname{sgn}_{j k}^{\rho}=\left\{\begin{array}{l}
+1: j \text { is on the right of } k \text { in } \rho(1,2, \ldots, n) \\
-1: j \text { is on the left of } k \text { in } \rho(1,2, \ldots, n)
\end{array}\right.
$$

the modification of (A.5) by degenerations of $g_{j_{i} k_{i}}^{(1)}$ can be written as [140]

$$
\begin{aligned}
\lim _{\tau \rightarrow i \infty} \frac{g_{j_{1} k_{1}}^{(1)} g_{j_{2} k_{2}}^{(1)} \ldots g_{j_{r} k_{r}}^{(1)}}{(2 \pi i)^{r} \sigma_{1} \sigma_{2} \ldots \sigma_{n}}= & \frac{(-1)^{n-1}}{2^{r}} \lim _{\sigma_{-} \rightarrow \infty}\left|\sigma_{-}\right|^{2} \\
& \times \sum_{\rho \in S_{n}} \operatorname{sgn}_{j_{1} k_{1}}^{\rho} \operatorname{sgn}_{j_{2} k_{2}}^{\rho} \ldots \operatorname{sgn}_{j_{r} k_{r}}^{\rho} \mathrm{PT}(+, \rho(1,2, \ldots, n),-) .
\end{aligned}
$$

The contributions of $(2 \pi i)^{-2 k} g_{j k}^{(2 k)}$ in turn degenerate to rational constants by (A.4) which multiply the overall sum over permutations $\rho$. Hence, (A.7) allows to straightforwardly expand the left-hand side of (A.1) in terms of Parke-Taylor factors in an $n$ !-element basis of PT( $+, \ldots,-)$. Matching the Parke-Taylor coefficients with those on the right-hand side determines the $c_{w, \gamma}$ in (3.37). It is a special property of the elliptic functions $V_{w}$ that their degeneration conspires to the cyclic combinations $\mathrm{PT}^{(1)}$ in (A.2), i.e. that the $(n-1)$ ! independent $c_{w, \gamma}$ are sufficient to accommodate the $n$ ! permutations of $\mathrm{PT}(+, 1,2, \ldots, n,-)$ in $1,2, \ldots, n$. 
For instance, the decompositions in (3.39) to (3.41) follow from the special cases of (A.7)

$$
\begin{aligned}
\lim _{\tau \rightarrow i \infty} \frac{V_{1}(1,2,3 \mid \tau)}{(2 \pi i) \sigma_{1} \sigma_{2} \sigma_{3}}= & \frac{1}{2} \lim _{\sigma_{-} \rightarrow \infty}\left|\sigma_{-}\right|^{2} \sum_{\rho \in S_{3}} \mathrm{PT}(+, \rho(1,2,3),-) \\
& \times\left(\operatorname{sgn}_{12}^{\rho}+\operatorname{sgn}_{23}^{\rho}+\operatorname{sgn}_{31}^{\rho}\right) \\
\lim _{\tau \rightarrow i \infty} \frac{V_{1}(1,2,3,4 \mid \tau)}{(2 \pi i) \sigma_{1} \sigma_{2} \sigma_{3} \sigma_{4}}= & -\frac{1}{2} \lim _{\sigma-\rightarrow}\left|\sigma_{-}\right|^{2} \sum_{\rho \in S_{4}} \mathrm{PT}(+, \rho(1,2,3,4),-) \\
\lim _{\tau \rightarrow i \infty} \frac{V_{2}(1,2,3,4 \mid \tau)}{(2 \pi i)^{2} \sigma_{1} \sigma_{2} \sigma_{3} \sigma_{4}}= & \left.-\lim _{\sigma_{-} \rightarrow \infty}\left|\sigma_{-}\right|^{2} \sum_{\rho \in S_{4}} \operatorname{PT}_{12}^{\rho}+\operatorname{sgn}_{23}^{\rho}+\operatorname{sgn}_{34}^{\rho}+\operatorname{sgn}_{41}^{\rho}\right) \\
& \times\left\{\frac{1}{3}+\frac{1}{4}\left(\operatorname{sgn}_{12}^{\rho} \operatorname{sgn}_{34}^{\rho}+\operatorname{sgn}_{23}^{\rho} \operatorname{sgn}_{41}^{\rho}\right)\right. \\
& +\frac{1}{4}\left(\operatorname{sgn}_{12}^{\rho} \operatorname{sgn}_{23}^{\rho}+\operatorname{sgn}_{23}^{\rho} \operatorname{sgn}_{34}^{\rho}+\operatorname{sgn}_{34}^{\rho} \operatorname{sgn}_{41}^{\rho}\right. \\
& \left.\left.+\operatorname{sgn}_{41}^{\rho} \operatorname{sgn}_{12}^{\rho}\right)\right\}
\end{aligned}
$$

once the right-hand sides are matched with the combinations of one-loop Parke-Taylor factors $\mathrm{PT}^{(1)}$ in (A.1) and (A.2).

\section{Appendix $B$. The initial value $\widehat{B}_{\eta_{2}, \eta_{3}}^{i \infty}$ at three points}

This appendix gathers the three-point initial values $\widehat{B}_{\eta_{2}, \eta_{3}}^{i \infty}(2,3 \mid \rho(2,3))$ for the $\alpha^{\prime}$-expansion (4.9) of B-cycle integrals up to and including weight four. The corresponding orders of $\widehat{J}_{\eta_{2}, \eta_{3}}^{i \infty}(2,3 \mid \rho(2,3))$ relevant to the $\alpha^{\prime}$-expansion (4.19) of $J$-integrals are obtained from the single-valued map $\left(\zeta_{2}, \zeta_{3}, \zeta_{4}\right) \rightarrow\left(0,2 \zeta_{3}, 0\right)$. Since even (odd) orders in the $\eta_{j}$-expansion integrate to zero on the odd (even) integration cycles $\mathfrak{B}(2,3) \pm \mathfrak{B}(3,2)$, we will separate the two types of contributions in order to infer $\widehat{B}_{\eta_{2}, \eta_{3}}^{i \infty}(2,3 \mid 3,2)$ from a relabeling of $\widehat{B}_{\eta_{2}, \eta_{3}}^{i \infty}(2,3 \mid 2,3)$.

The expressions in this appendix along with various higher-order terms in the $s_{i j^{-}}$and $\eta_{j}$-expansions can be found in the supplementary file at https://stacks.iop.org/A/55/025401/ mmedia, also see (4.27) for the appearance of $\zeta_{3,5}$.

\section{B.1. Even orders in $\eta_{j}$}

The terms of even orders in $\eta_{j}$ in the three-point initial values are given by

$$
\begin{aligned}
\left.\widehat{B}_{\eta_{2}, \eta_{3}}^{i \infty}(2,3 \mid 2,3)\right|_{\text {even }}= & \frac{1}{\eta_{23} \eta_{3}}\left(\frac{1}{2}+\frac{\zeta_{2}}{12}\left(s_{12}^{2}+s_{13}^{2}+s_{23}^{2}\right)+\frac{\zeta_{3}}{24}\left(s_{12}^{3}+s_{13}^{3}+s_{23}^{3}\right)\right. \\
& +\zeta_{4}\left[\frac{131}{1440}\left(s_{12}^{4}+s_{13}^{4}+s_{23}^{4}\right)+\frac{5}{144}\left(s_{12}^{2} s_{13}^{2}+s_{12}^{2} s_{23}^{2}+s_{23}^{2} s_{13}^{2}\right)\right. \\
& \left.\left.+\frac{1}{18} s_{12} s_{13} s_{23} s_{123}\right]+\ldots\right)
\end{aligned}
$$




$$
\begin{aligned}
& +\frac{\eta_{23}}{\eta_{3}}\left(-\zeta_{2}-\frac{\zeta_{3}}{2} s_{12}-\zeta_{4}\left[\frac{29 s_{12}^{2}}{24}+\frac{5 s_{13}^{2}}{12}+\frac{s_{13} s_{23}}{3}+\frac{5 s_{23}^{2}}{12}\right]+\ldots\right) \\
& +\frac{\eta_{3}}{\eta_{23}}\left(-\zeta_{2}-\frac{\zeta_{3}}{2} s_{23}-\zeta_{4}\left[\frac{29 s_{23}^{2}}{24}+\frac{5 s_{12}^{2}}{12}+\frac{s_{12} s_{13}}{3}+\frac{5 s_{13}^{2}}{12}\right]+\ldots\right) \\
& +\left(\frac{3 \zeta_{2} s_{13}}{s_{123}}+\frac{\zeta_{3}}{2} s_{13}+\frac{15 \zeta_{4} s_{13}}{4 s_{123}}\left(s_{12}^{2}+s_{12} s_{23}+s_{23}^{2}\right)\right. \\
& \left.-\frac{41}{24} \zeta_{4}\left(s_{12}+s_{23}\right) s_{13}+\frac{49 \zeta_{4} s_{13}^{2}}{24}+\ldots\right) \\
& +\eta_{23} \eta_{3}\left(5 \zeta_{4}\right)-\frac{\eta_{23}^{3}}{\eta_{3}} \zeta_{4}-\frac{\eta_{3}^{3}}{\eta_{23}} \zeta_{4}+\ldots
\end{aligned}
$$

and

$$
\left.\widehat{B}_{\eta_{2}, \eta_{3}}^{i \infty}(2,3 \mid 3,2)\right|_{\text {even }}=\left.\left.\widehat{B}_{\eta_{2}, \eta_{3}}^{i \infty}(2,3 \mid 2,3)\right|_{\text {even }}\right|_{s_{12} \leftrightarrow \eta_{13}} ^{\eta_{2} \leftrightarrow \eta_{3}}
$$

with MZVs of weight $\geqslant 5$ in the ellipsis.

\section{B.2. Odd orders in $\eta_{j}$}

The terms of odd orders in $\eta_{j}$ in the three-point initial values are given by

$$
\begin{aligned}
\left.\widehat{B}_{\eta_{2}, \eta_{3}}^{i \infty}(2,3 \mid 2,3)\right|_{\text {odd }}= & \frac{1}{\eta_{3}}\left(\frac{1}{s_{12}}+\frac{\zeta_{2} s_{123}^{2}}{6 s_{12}}+\zeta_{3}\left[\frac{s_{13} s_{23}}{4}+\frac{s_{123}^{3}}{12 s_{12}}\right]\right. \\
& \left.+\zeta_{4}\left[\frac{131 s_{123}^{4}}{720 s_{12}}-\frac{s_{23} s_{123} s_{13}}{20}\right]+\ldots\right) \\
& +\frac{1}{\eta_{23}}\left(\frac{1}{s_{23}}+\frac{\zeta_{2} s_{123}^{2}}{6 s_{23}}+\zeta_{3}\left[\frac{s_{12} s_{13}}{4}+\frac{s_{123}^{3}}{12 s_{23}}\right]\right. \\
& \left.+\zeta_{4}\left[\frac{131 s_{123}^{4}}{720 s_{23}}-\frac{s_{12} s_{123} s_{13}}{20}\right]+\ldots\right) \\
& +\eta_{3}\left(-\frac{2 \zeta_{2}}{s_{12}}-\frac{\zeta_{3} s_{123}}{s_{12}}+\zeta_{4}\left[-\frac{29 s_{123}^{2}}{12 s_{12}}+\frac{s_{13}}{4}\right]+\ldots\right) \\
& +\eta_{23}\left(-\frac{2 \zeta_{2}}{s_{23}}-\frac{\zeta_{3} s_{123}}{s_{23}}+\zeta_{4}\left[-\frac{29 s_{123}^{2}}{12 s_{23}}+\frac{s_{13}}{4}\right]+\ldots\right) \\
& +\frac{\eta_{23}^{2}}{\eta_{3}}\left(-\zeta_{3}+\zeta_{4}\left[\frac{2}{3} s_{123}+\frac{s_{12}}{4}\right]+\ldots\right) \\
& +\frac{\eta_{3}^{2}}{\eta_{23}}\left(-\zeta_{3}+\zeta_{4}\left[\frac{2}{3} s_{123}+\frac{s_{23}}{4}\right]+\ldots\right) \\
& +\eta_{23}^{3}\left(-\frac{2 \zeta_{4}}{s_{23}}+\ldots\right)+\eta_{3}^{3}\left(-\frac{2 \zeta_{4}}{s_{12}}+\ldots\right)+\ldots \quad \text { (B.3) }
\end{aligned}
$$

and

$$
\left.\widehat{B}_{\eta_{2}, \eta_{3}}^{i \infty}(2,3 \mid 3,2)\right|_{\text {odd }}=-\left.\left.\left.\widehat{B}_{\eta_{2}, \eta_{3}}^{i \infty}(2,3 \mid 2,3)\right|_{\text {odd }}\right|_{s_{12} \leftrightarrow s_{13}}\right|_{\eta_{2} \leftrightarrow \eta_{3}},
$$


again with MZVs of weight $\geqslant 5$ in the ellipsis.

\section{Appendix C. Examples of single-valued eMZVs}

\section{C.1. Systematics at depth one}

The simplest examples (5.12) and (5.13) of single-valued eMZVs extracted from the twopoint integrals (5.4) are special cases of the SV map (4.30) on holomorphic iterated Eisenstein integrals. For their depth-one combinations $\beta\left[\begin{array}{l}j \\ k\end{array}\right]$ in (4.10), the SV image $\beta^{\text {sv }}\left[\begin{array}{c}j \\ k\end{array}\right]$ yields the following Cauchy-Riemann derivatives $\nabla=2 i(\operatorname{Im} \tau)^{2} \partial_{\tau}$ of non-holomorphic Eisenstein series [18]

$$
\begin{aligned}
\beta^{\mathrm{sv}}\left[\begin{array}{c}
k-1 \\
2 k
\end{array}\right] & =-\frac{[(k-1) !]^{2}}{(2 k-1) !} E_{k}+\frac{2 \zeta_{2 k-1}}{(2 k-1)(4 y)^{k-1}} \\
\beta^{\mathrm{sv}}\left[\begin{array}{c}
k-1+m \\
2 k
\end{array}\right] & =-\frac{(-4)^{m}(k-1) !(k-1-m) !(\pi \nabla)^{m} E_{k}}{(2 k-1) !}+\frac{2 \zeta_{2 k-1}}{(2 k-1)(4 y)^{k-1-m}} \\
\beta^{\mathrm{sv}}\left[\begin{array}{c}
k-1-m \\
2 k
\end{array}\right] & =-\frac{(k-1) !(k-1-m) !(\pi \bar{\nabla})^{m} E_{k}}{(-4)^{m}(2 k-1) ! y^{2 m}}+\frac{2 \zeta_{2 k-1}}{(2 k-1)(4 y)^{k-1+m}},
\end{aligned}
$$

also see (4.22). While the objects on the right-hand side are expressible in terms of the lattice sums $\left[\begin{array}{ll}a & 0 \\ b & 0\end{array}\right]$ in (2.27) via (2.28), the $\beta\left[\begin{array}{l}j \\ k\end{array}\right]$ are simple combinations of B-cycle eMZVs $\omega\left(0^{p}, k \mid-\frac{1}{\tau}\right)$, where $0^{p}$ stands for a sequence $0,0, \ldots, 0$ of $p$ successive zeros. On these grounds, $\beta^{\text {sv }}\left[\begin{array}{l}j \\ k\end{array}\right]=\mathrm{SV} \beta\left[\begin{array}{l}j \\ k\end{array}\right]$ translates into simple relations such as

$$
\begin{aligned}
& \mathrm{SV} \omega\left(0,2 k+1 \mid-\frac{1}{\tau}\right)=-\frac{(\tau-\bar{\tau})^{2 k+1}}{2 \pi i} \mathcal{C}\left[\begin{array}{cc}
2 k+1 & 0 \\
1 & 0
\end{array}\right], \quad k \geqslant 1 \\
& \mathrm{SV} \omega\left(0,0,2 k+2 \mid-\frac{1}{\tau}\right)=\frac{(\tau-\bar{\tau})^{2 k+2}}{(2 \pi i)^{2}} \mathcal{C}\left[\begin{array}{cc}
2 k+2 & 0 \\
2 & 0
\end{array}\right], \quad k \geqslant 0 \\
& \mathrm{SV}\left(\omega\left(0,0,0,2 k+3 \mid-\frac{1}{\tau}\right)-\frac{1}{6} \omega\left(0,2 k+3 \mid-\frac{1}{\tau}\right)\right)=-\frac{(\tau-\bar{\tau})^{2 k+3}}{(2 \pi i)^{3}} \mathcal{C}\left[\begin{array}{cc}
2 k+3 & 0 \\
3 & 0
\end{array}\right], \\
& \quad k \geqslant-1 \\
& \mathrm{SV}\left(\omega\left(0,0,0,0,2 k+4 \mid-\frac{1}{\tau}\right)-\frac{1}{6} \omega\left(0,0,2 k+4 \mid-\frac{1}{\tau}\right)\right)=\frac{(\tau-\bar{\tau})^{2 k+4}}{(2 \pi i)^{4}} \mathcal{C}\left[\begin{array}{cc}
2 k+4 & 0 \\
4 & 0
\end{array}\right], \\
& \quad k \geqslant-1
\end{aligned}
$$

as well as

$$
\begin{aligned}
\operatorname{SV} & \left(\omega\left(0,0,0,0,0,2 k+5 \mid-\frac{1}{\tau}\right)-\frac{1}{6} \omega\left(0,0,0,2 k+5 \mid-\frac{1}{\tau}\right)+\frac{7}{360} \omega\left(0,2 k+5 \mid-\frac{1}{\tau}\right)\right) \\
& =-\frac{(\tau-\bar{\tau})^{2 k+5}}{(2 \pi i)^{5}} \mathcal{C}\left[\begin{array}{cc}
2 k+5 & 0 \\
5 & 0
\end{array}\right], \quad k \geqslant-2 .
\end{aligned}
$$


The relative factors of $-\frac{1}{6}$ and $\frac{7}{360}$ among the eMZVs of different lengths are engineered to streamline the iterated-Eisenstein-integral representation [17] and generalize as follows [104]

$\mathrm{SV} \sum_{j=0}^{\ell-1} \frac{B_{j}}{j !} \omega\left(0^{\ell-j}, 2 k+\ell \mid-\frac{1}{\tau}\right)=(-1)^{\ell} \frac{(\tau-\bar{\tau})^{2 k+\ell}}{(2 \pi i)^{\ell}} \mathcal{C}\left[\begin{array}{cc}2 k+\ell & 0 \\ \ell & 0\end{array}\right], \quad k \geqslant 1-\left\lceil\frac{\ell}{2}\right\rceil, \quad \ell \geqslant 1$.

In obtaining (C.2) and (C.3) from (C.4), we have used SV $\omega(m)=0 \forall m \geqslant 1$ and the following simplifications of the only eMZV $\omega\left(0^{\ell-1}, 2 k+\ell \mid \tau \ell\right)$ whose length and weight adds up to an even number [17],

$$
\begin{aligned}
\omega(0,0,2 k+1 \mid \tau) & =\frac{1}{2} \omega(0,2 k+1) \\
\omega(0,0,0,2 k \mid \tau) & =\frac{1}{2} \omega(0,0,2 k)-\frac{1}{24} \omega(2 k) \\
\omega(0,0,0,0,2 k+1 \mid \tau) & =\frac{1}{2} \omega(0,0,0,2 k+1)-\frac{1}{24} \omega(0,2 k+1) .
\end{aligned}
$$

Based on the dictionary (2.28) between lattice sums $\left[\begin{array}{ll}a & 0 \\ b & 0\end{array}\right]$ and non-holomorphic Eisenstein series, one can reformulate (C.4) as

$\mathrm{SV} \sum_{j=0}^{\ell-1} \frac{B_{j}}{j !} \omega\left(0^{\ell-j}, 2 k+\ell \mid-\frac{1}{\tau}\right)=(-1)^{\ell} \frac{(k+\ell-1) !}{(2 k+\ell-1) !}(-4 \pi \nabla)^{k} E_{k+\ell}, \quad k \geqslant 0, \ell \geqslant 1$,

where $k=0$ needs to be excluded if $\ell=1$, for instance

$$
\begin{gathered}
\operatorname{SV} \omega\left(0,2 k+1 \mid-\frac{1}{\tau}\right)=-\frac{k !}{(2 k) !}(-4 \pi \nabla)^{k} E_{k+1}, \quad k \geqslant 1 \\
\operatorname{SV} \omega\left(0,0,2 k+2 \mid-\frac{1}{\tau}\right)=\frac{(k+1) !}{(2 k+1) !}(-4 \pi \nabla)^{k} E_{k+2}, \quad k \geqslant 0 .
\end{gathered}
$$

Moreover, by extending (C.4) to $k \rightarrow-k$ and applying the complex conjugate of (2.28), we also obtain antiholomorphic Cauchy-Riemann derivatives as single-valued eMZVs (with $\ell-2 k>0$ ),

$$
\begin{aligned}
\mathrm{SV} \sum_{j=0}^{\ell-1} \frac{B_{j}}{j !} \omega\left(0^{\ell-j}, \ell-2 k \mid-\frac{1}{\tau}\right) & =(-1)^{\ell} \frac{(\tau-\bar{\tau})^{\ell-2 k}}{(2 \pi i)^{\ell}} \mathcal{C}\left[\begin{array}{cc}
\ell-2 k & 0 \\
\ell & 0
\end{array}\right] \\
& =(-1)^{\ell+k} \frac{(\ell-k-1) !}{(\ell-1) !} \frac{(\pi \bar{\nabla})^{k} E_{\ell-k}}{(2 y)^{2 k}}
\end{aligned}
$$


The simplest examples include

$$
\begin{array}{r}
\operatorname{SV}\left(\omega\left(0,0,0,1 \mid-\frac{1}{\tau}\right)-\frac{1}{6} \omega\left(0,1 \mid-\frac{1}{\tau}\right)\right)=\frac{\pi \bar{\nabla} E_{2}}{8 y^{2}} \\
\operatorname{SV}\left(\omega\left(0,0,0,0,2 \mid-\frac{1}{\tau}\right)-\frac{1}{6} \omega\left(0,0,2 \mid-\frac{1}{\tau}\right)\right)=-\frac{\pi \bar{\nabla} E_{3}}{12 y^{2}} \\
\operatorname{SV}\left(\omega\left(0,0,0,0,0,1 \mid-\frac{1}{\tau}\right)-\frac{1}{6} \omega\left(0,0,0,1 \mid-\frac{1}{\tau}\right)+\frac{7}{360} \omega\left(0,1 \mid-\frac{1}{\tau}\right)\right)=-\frac{(\pi \bar{\nabla})^{2} E_{3}}{192 y^{4}} \\
\operatorname{SV}\left(\omega\left(0,0,0,0,0,3 \mid-\frac{1}{\tau}\right)-\frac{1}{6} \omega\left(0,0,0,3 \mid-\frac{1}{\tau}\right)+\frac{7}{360} \omega\left(0,3 \mid-\frac{1}{\tau}\right)\right)=\frac{\pi \bar{\nabla} E_{4}}{16 y^{2}},
\end{array}
$$

and the first two lines are equivalent to those in (5.32).

\section{C.2. Examples with real MGFs at depth two}

By inspecting the $s_{i j}^{4}$ order of the two-point integrals $B_{(0)}^{\tau}, J_{(0)}^{\tau}$ and the $s_{i j}^{3}$ order of $B_{(2)}^{\tau}, J_{(2)}^{\tau}$, we have obtained the representations (5.14) of $E_{2,2}$ and $\pi \nabla E_{2,2}$ as single-valued eMZVs. One can extract similar representations for $E_{2,3}, \pi \nabla E_{2,3}$ and $(\pi \nabla)^{2} E_{2,3}$ from the $s_{i j}^{5}$ order of $B_{(0)}^{\tau}, J_{(0)}^{\tau}$, the $s_{i j}^{4}$ order of $B_{(2)}^{\tau}, J_{(2)}^{\tau}$ and the $s_{i j}^{3}$ order of $B_{(4)}^{\tau}, J_{(4)}^{\tau}$, respectively:

$$
\begin{aligned}
E_{2,3}= & \operatorname{SV}\left(-\frac{167}{35} \omega\left(0,0,0,0,0,5 \mid-\frac{1}{\tau}\right)+2 \omega\left(0,0,0,0,1,4 \mid-\frac{1}{\tau}\right)\right. \\
& +\frac{97}{210} \omega\left(0,0,0,5 \mid-\frac{1}{\tau}\right)-\frac{1}{3} \omega\left(0,0,2,3 \mid-\frac{1}{\tau}\right) \\
& \left.+2 \omega\left(0,0,0,0,2 \mid-\frac{1}{\tau}\right) \omega\left(0,3 \mid-\frac{1}{\tau}\right)+\frac{7}{200} \omega\left(0,5 \mid-\frac{1}{\tau}\right)\right) \\
\pi \nabla E_{2,3}= & \operatorname{SV}\left(-\frac{1}{12} \omega\left(0,3 \mid-\frac{1}{\tau}\right)^{2}+\frac{13}{168} \omega\left(0,0,6 \mid-\frac{1}{\tau}\right)+\omega\left(0,3 \mid-\frac{1}{\tau}\right) \omega\left(0,0,0,3 \mid-\frac{1}{\tau}\right)\right. \\
& \left.-\frac{41}{28} \omega\left(0,0,0,0,6 \mid-\frac{1}{\tau}\right)+\frac{1}{2} \omega\left(0,0,0,2,4 \mid-\frac{1}{\tau}\right)\right) \\
(\pi \nabla)^{2} E_{2,3}= & \operatorname{SV}\left(\frac{25}{336} \omega\left(0,7 \mid-\frac{1}{\tau}\right)+\frac{5}{8} \omega\left(0,3 \mid-\frac{1}{\tau}\right) \omega\left(0,0,4 \mid-\frac{1}{\tau}\right)-\frac{23}{28} \omega\left(0,0,0,7 \mid-\frac{1}{\tau}\right)\right. \\
& \left.+\frac{1}{4} \omega\left(0,0,2,5 \mid-\frac{1}{\tau}\right)+\frac{1}{8} \omega\left(0,0,4,3 \mid-\frac{1}{\tau}\right)\right)
\end{aligned}
$$

The corresponding lattice-sum representations [51, 62] and $\beta^{\text {sv }}$ representations [18] are given by

$$
\begin{aligned}
E_{2,3}= & \left(\frac{\operatorname{Im} \tau}{\pi}\right)^{5}\left(\mathcal{C}\left[\begin{array}{lll}
3 & 1 & 1 \\
3 & 1 & 1
\end{array}\right]-\frac{43}{35} \mathcal{C}\left[\begin{array}{ll}
5 & 0 \\
5 & 0
\end{array}\right]\right) \\
= & -120 \beta^{\mathrm{sv}}\left[\begin{array}{ll}
2 & 1 \\
4 & 6
\end{array}\right]-120 \beta^{\mathrm{sv}}\left[\begin{array}{ll}
3 & 0 \\
6 & 4
\end{array}\right]+\frac{12 \zeta_{5}}{y} \beta^{\mathrm{sv}}\left[\begin{array}{l}
0 \\
4
\end{array}\right]+80 \zeta_{3} \beta^{\mathrm{sv}}\left[\begin{array}{l}
1 \\
6
\end{array}\right] \\
& -\frac{\zeta_{5}}{36}+\frac{7 \zeta_{7}}{16 y^{2}}-\frac{\zeta_{3} \zeta_{5}}{2 y^{3}}
\end{aligned}
$$




$$
\begin{aligned}
& \pi \nabla E_{2,3}=\frac{(\operatorname{Im} \tau)^{6}}{\pi^{4}}\left(3 \mathcal{C}\left[\begin{array}{lll}
1 & 1 & 4 \\
1 & 1 & 2
\end{array}\right]+2 \mathcal{C}\left[\begin{array}{lll}
1 & 2 & 3 \\
1 & 0 & 3
\end{array}\right]-\frac{43}{7} \mathcal{C}\left[\begin{array}{ll}
6 & 0 \\
4 & 0
\end{array}\right]\right) \\
& =90 \beta^{\mathrm{sv}}\left[\begin{array}{ll}
2 & 2 \\
4 & 6
\end{array}\right]+60 \beta^{\mathrm{sv}}\left[\begin{array}{ll}
3 & 1 \\
6 & 4
\end{array}\right]+30 \beta^{\mathrm{sv}}\left[\begin{array}{ll}
4 & 0 \\
6 & 4
\end{array}\right] \\
& -60 \zeta_{3} \beta^{\mathrm{sv}}\left[\begin{array}{l}
2 \\
6
\end{array}\right]-12 \zeta_{5} \beta^{\mathrm{sv}}\left[\begin{array}{l}
0 \\
4
\end{array}\right]-\frac{6 \zeta_{5}}{y} \beta^{\mathrm{sv}}\left[\begin{array}{l}
1 \\
4
\end{array}\right]-\frac{7 \zeta_{7}}{8 y}+\frac{3 \zeta_{3} \zeta_{5}}{2 y^{2}} \\
& (\pi \nabla)^{2} E_{2,3}=\frac{(\operatorname{Im} \tau)^{7}}{\pi^{3}}\left(4 \mathcal{C}\left[\begin{array}{lll}
0 & 2 & 5 \\
1 & 0 & 2
\end{array}\right]-4 \mathcal{C}\left[\begin{array}{ll}
3 & 0 \\
1 & 0
\end{array}\right] \mathcal{C}\left[\begin{array}{ll}
4 & 0 \\
2 & 0
\end{array}\right]-\frac{62}{7} \mathcal{C}\left[\begin{array}{ll}
7 & 0 \\
3 & 0
\end{array}\right]\right) \\
& =-45 \beta^{\mathrm{sv}}\left[\begin{array}{ll}
2 & 3 \\
4 & 6
\end{array}\right]-15 \beta^{\mathrm{sv}}\left[\begin{array}{ll}
3 & 2 \\
6 & 4
\end{array}\right]-30 \beta^{\mathrm{sv}}\left[\begin{array}{ll}
4 & 1 \\
6 & 4
\end{array}\right] \\
& +30 \zeta_{3} \beta^{\mathrm{sv}}\left[\begin{array}{l}
3 \\
6
\end{array}\right]+12 \zeta_{5} \beta^{\mathrm{sv}}\left[\begin{array}{l}
1 \\
4
\end{array}\right]+\frac{3 \zeta_{5}}{2 y} \beta^{\mathrm{sv}}\left[\begin{array}{l}
2 \\
4
\end{array}\right]+\frac{7 \zeta_{7}}{8}-\frac{3 \zeta_{3} \zeta_{5}}{y} \text {. }
\end{aligned}
$$

\section{ORCID iDs}

Jan E Gerken (D) https://orcid.org/0000-0002-0172-7944

Axel Kleinschmidt (D) https://orcid.org/0000-0003-3143-2239

Carlos R Mafra (D) https://orcid.org/0000-0001-9842-9654

Oliver Schlotterer (D) https://orcid.org/0000-0002-1048-661X

Bram Verbeek (D) https://orcid.org/0000-0002-4210-396X

\section{References}

[1] Levin A 1997 Elliptic polylogarithms: an analytic theory Compos. Math. 106 267-82

[2] Brown F and Levin A 2011 Multiple elliptic polylogarithms (arXiv:1110.6917 [math])

[3] Enriquez B 2016 Analogues elliptiques des nombres multizétas Bull. Soc. Math. France 144 395-427

[4] Broedel J, Mafra C R, Matthes N and Schlotterer O 2015 Elliptic multiple zeta values and one-loop superstring amplitudes J. High Energy Phys. JHEP07(2015)112

[5] Broedel J, Matthes N, Richter G and Schlotterer O 2018 Twisted elliptic multiple zeta values and non-planar one-loop open-string amplitudes J. Phys. A: Math. Theor. 51285401

[6] Broedel J and Schlotterer O 2019 One-loop string scattering amplitudes as iterated Eisenstein integrals Proc., KMPB Conf.: Elliptic Integrals, Elliptic Functions and Modular Forms in Quantum Field Theory (Zeuthen, Germany October 23-26, 2017) pp 133-59

[7] Green M B and Vanhove P 2000 The low-energy expansion of the one loop type II superstring amplitude Phys. Rev. D 61104011

[8] Green M B, Russo J G and Vanhove P 2008 Low energy expansion of the four-particle genus-one amplitude in type II superstring theory J. High Energy Phys. JHEP02(2008)020

[9] D'Hoker E, Green M B and Vanhove P 2015 On the modular structure of the genus-one type II superstring low energy expansion J. High Energy Phys. JHEP08(2015)041

[10] D’Hoker E, Green M B, Gürdogan Ö and Vanhove P 2017 Modular graph functions Commun. Num. Theor. Phys. 11 165-218

[11] D'Hoker E and Green M B 2018 Identities between modular graph forms J. Number Theor. 189 $25-80$

[12] Zerbini F 2016 Single-valued multiple zeta values in genus 1 superstring amplitudes Commun. Num. Theor. Phys. $10703-37$

[13] Brown F 2018 A class of non-holomorphic modular forms I Res. Math. Sci. 55 
[14] Brown F 2020 A class of non-holomorphic modular forms II: equivariant iterated Eisenstein integrals Forum Math. Sigma 81

[15] Panzer E 2018 Modular graph functions as iterated Eisenstein integrals Elliptic Integrals in Mathematics and Physics (Ascona, Switzerland) https://indico.cern.ch/event/700233/contributions/ 3112451/attachments/1712442/2761239/elliptic.pdf

[16] Zagier D and Zerbini F 2020 Genus-zero and genus-one string amplitudes and special multiple zeta values Commun. Num. Theor. Phys. 14 413-52

[17] Broedel J, Matthes N and Schlotterer O 2016 Relations between elliptic multiple zeta values and a special derivation algebra J. Phys. A: Math. Theor. 49155203

[18] Gerken J E, Kleinschmidt A and Schlotterer O 2020 Generating series of all modular graph forms from iterated Eisenstein integrals J. High Energy Phys. JHEP07(2020)190

[19] Bloch S and Vanhove P 2015 The elliptic dilogarithm for the sunset graph J. Number Theory 148 328-64

[20] Bloch S, Kerr M and Vanhove P 2015 A Feynman integral via higher normal functions Compos. Math. 151 2329-75

[21] Adams L and Weinzierl S 2018 Feynman integrals and iterated integrals of modular forms Commun. Num. Theor. Phys. 12 193-251

[22] Ablinger J, Blümlein J, De Freitas A, van Hoeij M, Imamoglu E, Raab C G, Radu C-S and Schneider C 2018 Iterated elliptic and hypergeometric integrals for Feynman diagrams J. Math. Phys. 59062305

[23] Remiddi E and Tancredi L 2017 An elliptic generalization of multiple polylogarithms Nucl. Phys. B $925212-51$

[24] Bourjaily J L, McLeod A J, Spradlin M, von Hippel M and Wilhelm M 2018 Elliptic double-box integrals: massless scattering amplitudes beyond polylogarithms Phys. Rev. Lett. 120121603

[25] Broedel J, Duhr C, Dulat F and Tancredi L 2018 Elliptic polylogarithms and iterated integrals on elliptic curves: I. General formalism J. High Energy Phys. JHEP05(2018)093

[26] Broedel J, Duhr C, Dulat F and Tancredi L 2018 Elliptic polylogarithms and iterated integrals on elliptic curves II: an application to the sunrise integral Phys. Rev. D 97116009

[27] Adams L and Weinzierl S 2018 The $\varepsilon$-form of the differential equations for Feynman integrals in the elliptic case Phys. Lett. B 781 270-8

[28] Broedel J, Duhr C, Dulat F, Penante B and Tancredi L 2018 Elliptic symbol calculus: from elliptic polylogarithms to iterated integrals of Eisenstein series J. High Energy Phys. JHEP08(2018)014

[29] Adams L, Chaubey E and Weinzierl S 2018 Planar double box integral for top pair production with a closed top loop to all orders in the dimensional regularization parameter Phys. Rev. Lett. 121 142001

[30] Adams L, Chaubey E and Weinzierl S 2018 Analytic results for the planar double box integral relevant to top-pair production with a closed top loop J. High Energy Phys. JHEP10(2018)206

[31] Broedel J, Duhr C, Dulat F, Penante B and Tancredi L 2018 Elliptic polylogarithms and two-loop Feynman integrals PoS LL2018 005

[32] Blümlein J, De Freitas A, Van Hoeij M, Imamoglu E, Marquard P and Schneider C 2018 The $\rho$ parameter at three loops and elliptic integrals PoS LL2018 017

[33] Broedel J, Duhr C, Dulat F, Penante B and Tancredi L 2019 Elliptic polylogarithms and Feynman parameter integrals J. High Energy Phys. JHEP05(2019)120

[34] Bogner C, Müller-Stach S and Weinzierl S 2020 The unequal mass sunrise integral expressed through iterated integrals on $\overline{\mathcal{M}}_{1,3}$ Nucl. Phys. B 954114991

[35] Broedel J, Duhr C, Dulat F, Marzucca R, Penante B and Tancredi L 2019 An analytic solution for the equal-mass banana graph J. High Energy Phys. JHEP09(2019)112

[36] Duhr C and Tancredi L 2020 Algorithms and tools for iterated Eisenstein integrals J. High Energy Phys. JHEP02(2020)105

[37] Abreu S, Becchetti M, Duhr C and Marzucca R 2020 Three-loop contributions to the $\rho$ parameter and iterated integrals of modular forms J. High Energy Phys. JHEP02(2020)050

[38] Bogner C, Hönemann I, Tempest K, Schweitzer A and Weinzierl S 2020 Numerics for elliptic Feynman integrals CERN Yellow Reports: Monographs 3 177-84

[39] Walden M and Weinzierl S 2020 Numerical evaluation of iterated integrals related to elliptic Feynman integrals (arXiv:2010.05271 [hep-ph])

[40] Schlotterer O and Stieberger S 2013 Motivic multiple zeta values and superstring amplitudes $J$. Phys. A: Math. Theor. 46475401 
[41] Stieberger S 2014 Closed superstring amplitudes, single-valued multiple zeta values and the Deligne associator J. Phys. A: Math. Theor. 47155401

[42] Stieberger $\mathrm{S}$ and Taylor T R 2014 Closed string amplitudes as single-valued open string amplitudes Nucl. Phys. B 881 269-87

[43] Schlotterer O and Schnetz O 2019 Closed strings as single-valued open strings: a genus-zero derivation J. Phys. A: Math. Theor. 52045401

[44] Vanhove P and Zerbini F 2018 Closed string amplitudes from single-valued correlation functions (arXiv:1812.03018 [hep-th])

[45] Brown F and Dupont C 2019 Single-valued integration and superstring amplitudes in genus zero (arXiv:1910.01107 [math.NT])

[46] Brown F 2014 Single-valued motivic periods and multiple zeta values Forum Math. Sigma 2 e25

[47] Brown F 2015 Notes on motivic periods Commun. Num. Theor. Phys. 11 557-655

[48] Schnetz O 2014 Graphical functions and single-valued multiple polylogarithms Commun. Num. Theor. Phys. 8 589-675

[49] Brown F and Dupont C 2018 Single-valued integration and double copy (arXiv:1810.07682 [math.NT])

[50] Brown F C S 2004 Polylogarithmes multiples uniformes en une variable C. R. Math. 338 527-32

[51] Broedel J, Schlotterer O and Zerbini F 2019 From elliptic multiple zeta values to modular graph functions: open and closed strings at one loop J. High Energy Phys. JHEP01(2019)155

[52] Gerken J E, Kleinschmidt A and Schlotterer O 2019 Heterotic-string amplitudes at one loop: modular graph forms and relations to open strings J. High Energy Phys. JHEP01(2019)052

[53] D'Hoker E and Duke W 2018 Fourier series of modular graph functions J. Number Theory 192 $1-36$

[54] D'Hoker E and Kaidi J 2019 Modular graph functions and odd cuspidal functions. Fourier and Poincaré series J. High Energy Phys. JHEP04(2019)136

[55] D'Hoker E and Green M B 2020 Absence of irreducible multiple zeta-values in melon modular graph functions Commun. Num. Theor. Phys. 14 315-24

[56] Vanhove P and Zerbini F 2020 Building blocks of closed and open string amplitudes MathemAmplitudes 2019: Intersection Theory and Feynman Integrals $\mathrm{p} 7$

[57] Beilinson A, Varchenko A, Goncharov A and Shekhtman V 1991 Projective geometry and Ktheory Leningr. Math. J. 3 523-75

[58] Schnetz O 2018 Graphical hyperlogarithms Number Theory, Algebraic Geometry and Physics (Bonn: HIM)

[59] Mafra C R and Schlotterer O 2020 'All-order alpha'-expansion of one-loop open-string integrals Phys. Rev. Lett. 124101603

[60] Mafra C R and Schlotterer O 2020 One-loop open-string integrals from differential equations: 'all-order $\alpha$ '-expansions at $n$ points J. High Energy Phys. JHEP03(2020)007

[61] Gerken J E, Kleinschmidt A and Schlotterer O 2020 All-order differential equations for one-loop closed-string integrals and modular graph forms J. High Energy Phys. JHEP01(2020)064

[62] Gerken J E 2021 Basis decompositions and a mathematica package for modular graph forms J. Phys. A $\mathbf{5 4} 195401$

[63] Mafra C R, Schlotterer O and Stieberger S 2013 Complete $N$-point superstring disk amplitude: I. Pure spinor computation Nucl. Phys. B 873 419-60

[64] Azevedo T, Chiodaroli M, Johansson H and Schlotterer O 2018 Heterotic and bosonic string amplitudes via field theory J. High Energy Phys. JHEP10(2018)012

[65] Broedel J, Schlotterer O and Stieberger S 2013 Polylogarithms, multiple zeta values and superstring amplitudes Fortschr. Phys. 61 812-70

[66] Bjerrum-Bohr N E J, Damgaard P H and Vanhove P 2009 Minimal basis for gauge theory amplitudes Phys. Rev. Lett. 103161602

[67] Stieberger S 2009 Open \& closed vs pure open string disk amplitudes (arXiv:0907.2211 [hep-th])

[68] Terasoma T 2002 Selberg integrals and multiple zeta values Compos. Math. 133 1-24

[69] Brown F C S 2009 Multiple zeta values and periods of moduli spaces $\mathcal{M}_{0, n}(\mathbb{R})$ Ann. Sci. École Norm. Sup. 42371

[70] Broedel J, Schlotterer O, Stieberger S and Terasoma T 2014 All order $\alpha^{\prime}$-expansion of superstring trees from the Drinfeld associator Phys. Rev. D 89066014

[71] Mafra C R and Schlotterer O 2017 Non-abelian Z-theory: Berends-Giele recursion for the $\alpha^{\prime}$ expansion of disk integrals J. High Energy Phys. JHEP01(2017)031

[72] Broedel J, Schlotterer O and Stieberger S 2013 http://mzv.mpp.mpg.de 
[73] Mafra C and Schlotterer O 2016 https://repo.or.cz/BGap.git

[74] Brown F 2012 Mixed tate motives over $\mathbb{Z}$ Ann. Math. 175 949-76

[75] Polchinski J 2007 String Theory. Vol. 1: An introduction to the Bosonic String (Cambridge Monographs on Mathematical Physics) (Cambridge: Cambridge University Press)

[76] Green M B, Schwarz J H and Witten E 1988 Superstring Theory. Vol. 2: Loop Amplitudes, Anomalies and Phenomenology (Cambridge: Cambridge University Press)

[77] Zerbini F 2018 Modular and holomorphic graph function from superstring amplitudes $K M P B$ Conf.: Elliptic Integrals, Elliptic Functions and Modular Forms in Quantum Field Theory (Zeuthen, Germany October 23-26, 2017)

[78] Kronecker L 1881 Zur Theorie der elliptischen Funktionen Math. Werke IV 313-8

[79] Dolan L and Goddard P 2009 Current algebra on the torus Commun. Math. Phys. 285 219-64

[80] Green M B, Schwarz J H and Brink L $1982 N=4$ Yang-Mills and $N=8$ supergravity as limits of string theories Nucl. Phys. B 198 474-92

[81] Tsuchiya A 1989 More on one-loop massless amplitudes of superstring theories Phys. Rev. D 39 1626

[82] Mafra C R and Schlotterer O 2019 Towards the $n$-point one-loop superstring amplitude: III. Oneloop correlators and their double-copy structure J. High Energy Phys. JHEP08(2019)092

[83] Tsunogai H 1995 On some derivations of Lie algebras related to Galois representations Publ. Res. Inst. Math. Sci. 31 113-34

[84] Luque J-G, Novelli J-C and Thibon J-Y 2007 Period polynomials and Ihara brackets J. Lie Theory 17 229-39

[85] Pollack A 2009 Relations between derivations arising from modular forms Undergraduate Thesis Duke University https://dukespace.lib.duke.edu/dspace/handle/10161/1281

[86] Broedel J and Kaderli A 2019 Amplitude recursions with an extra marked point (arXiv:1912.09927 [hep-th])

[87] Broedel J, Kaderli A and Schlotterer O 2020 Two dialects for KZB equations: generating one-loop open-string integrals J. High Energy Phys. JHEP12(2020)036

[88] D'Hoker E and Kaidi J 2016 Hierarchy of modular graph identities J. High Energy Phys. JHEP11(2016)051

[89] D’Hoker E, Green M B and Vanhove P 2018 Proof of a modular relation between 1-, 2- and 3-loop Feynman diagrams on a torus J. Number Theory 196381

[90] Basu A 2016 Proving relations between modular graph functions Class. Quantum Grav. 33235011

[91] Matthes N 2017 On the algebraic structure of iterated integrals of quasimodular forms Algebra Number Theory $112113-30$

[92] Broedel J, Matthes N and Schlotterer O 2015 https://tools.aei.mpg.de/emzv

[93] Zerbini F 2017 Elliptic multiple zeta values, modular graph functions and genus 1 superstring scattering amplitudes $P h D$ Thesis Bonn University

[94] Geyer Y, Mason L, Monteiro R and Tourkine P 2015 Loop integrands for scattering amplitudes from the Riemann sphere Phys. Rev. Lett. 115121603

[95] Kleinschmidt A, Schlotterer O and Verbeek B in preparation

[96] Vermaseren J A M 2000 New features of FORM (arXiv:math-ph/0010025 [math-ph])

[97] Mafra C R 2020 Constant terms of B-cycle eMZVs www.southampton.ac.uk/ crm1n16/ Bcycle.html

[98] Mumford D, Musili C, Nori M, Previato E and Stillman M 2007 Tata Lectures on Theta. I (Modern Birkhäuser Classics) (Boston, MA: Birkhäuser)

[99] Mafra C R and Schlotterer O 2019 Towards the $n$-point one-loop superstring amplitude: II. Worldsheet functions and their duality to kinematics J. High Energy Phys. JHEP08(2019)091

[100] Kleiss R and Kuijf H 1989 Multigluon cross sections and 5-jet production at hadron colliders Nucl. Phys. B 312 616-44

[101] Brown F 2014 Multiple modular values and the relative completion of the fundamental group of $\mathcal{M}_{1,1}$ (arXiv:1407.5167 [math.NT])

[102] Blümlein J, Broadhurst D J and Vermaseren J A M 2010 The multiple zeta value data mine Comput. Phys. Commun. 181 582-625

[103] Panzer E 2019 Modular graph functions as iterated Eisenstein integrals HET-Discovery Seminar (Niels Bohr Institute, Copenhagen, Denmark) https://nbi.ku.dk/english/research/theoreticalparticle-physics-and-cosmology/calendar/2019/het-discovery-seminar-erik-panzeri/

[104] Matthes N 2017 private communication 
[105] Richards D M 2008 The one-loop five-graviton amplitude and the effective action J. High Energy Phys. JHEP10(2008)042

[106] Green M B, Mafra C R and Schlotterer O 2013 Multiparticle one-loop amplitudes and S-duality in closed superstring theory J. High Energy Phys. JHEP10(2013)188

[107] Saad A 2020 Multiple zeta values and iterated Eisenstein integrals (arXiv:2009.09885 [math.NT])

[108] Basu A 2016 Poisson equation for the three-loop ladder diagram in string theory at genus one Int. J. Mod. Phys. A 311650169

[109] Kleinschmidt A and Verschinin V 2017 Tetrahedral modular graph functions J. High Energy Phys. JHEP09(2017)155

[110] Basu A 2017 Low momentum expansion of one loop amplitudes in heterotic string theory J. High Energy Phys. JHEP11(2017)139

[111] Basu A 2019 Eigenvalue equation for the modular graph $C_{a, b, c, d}$ J. High Energy Phys. JHEP07(2019)126

[112] D'Hoker E and Green M B 2019 Exploring transcendentality in superstring amplitudes J. High Energy Phys. JHEP07(2019)149

[113] Angelantonj C, Florakis I and Pioline B 2012 A new look at one-loop integrals in string theory Commun. Num. Theor. Phys. 6 159-201

[114] Angelantonj C, Florakis I and Pioline B 2012 One-loop BPS amplitudes as BPS-state sums J. High Energy Phys. JHEP06(2012)070

[115] Ahlén O and Kleinschmidt A $2018 D^{6} R^{4}$ curvature corrections, modular graph functions and Poincaré series J. High Energy Phys. JHEP05(2018)194

[116] Dorigoni D and Kleinschmidt A 2019 Modular graph functions and asymptotic expansions of Poincaré series Commun. Num. Theor. Phys. 13 569-617

[117] D'Hoker E 2019 Integral of two-loop modular graph functions J. High Energy Phys. JHEP06(2019)092

[118] Dorigoni D and Kleinschmidt A 2020 Resurgent expansion of Lambert series and iterated Eisenstein integrals (arXiv:2001.11035 [hep-th])

[119] Green M B and Schwarz J H 1982 Supersymmetric dual string theory Nucl. Phys. B 198 441-460

[120] Angelantonj C and Sagnotti A 2002 Open strings Phys. Rep. 371 1-150 Angelantonj C and Sagnotti A 2003 Phys. Rep. 376407 (erratum)

[121] Lüst D and Stieberger S 2007 Gauge threshold corrections in intersecting brane world models Fortschr. Phys. $\mathbf{5 5}$ 427-65

[122] Hohenegger S and Stieberger S 2017 Monodromy relations in higher-loop string amplitudes Nucl. Phys. B 925 63-134

[123] Broedel J and Kaderli A 2020 Functional relations for elliptic polylogarithms J. Phys. A: Math. Theor. $\mathbf{5 3} 245201$

[124] Kawai H, Lewellen D C and Tye S-H H 1986 A relation between tree amplitudes of closed and open strings Nucl. Phys. B 269 1-23

[125] Tourkine P and Vanhove P 2016 Higher-loop amplitude monodromy relations in string and gauge theory Phys. Rev. Lett. 117211601

[126] Ochirov A, Tourkine P and Vanhove P 2017 One-loop monodromy relations on single cuts J. High Energy Phys. JHEP10(2017)105

[127] Casali E, Mizera S and Tourkine P 2020 Loop amplitudes monodromy relations and colorkinematics duality (arXiv:2005.05329 [hep-th])

[128] Casali E, Mizera S and Tourkine P 2019 Monodromy relations from twisted homology J. High Energy Phys. JHEP12(2019)087

[129] D'Hoker E and Phong D H 1988 The geometry of string perturbation theory Rev. Mod. Phys. 60 917

[130] D'Hoker E and Phong D H 1989 Conformal scalar fields and chiral splitting on superriemann surfaces Commun. Math. Phys. 125469

[131] D'Hoker E, Green M B and Pioline B 2019 Higher genus modular graph functions, string invariants, and their exact asymptotics Commun. Math. Phys. 927 927-79

[132] D'Hoker E, Green M B and Pioline B 2019 Asymptotics of the $D^{8} \mathcal{R}^{4}$ genus-two string invariant Commun. Num. Theor. Phys. 13 351-462

[133] D'Hoker E and Schlotterer O 2020 Identities among higher genus modular graph tensors (arXiv:2010.00924 [hep-th])

[134] D'Hoker E and Phong D 2005 Two-loop superstrings VI: non-renormalization theorems and the 4-point function Nucl. Phys. B 715 3-90 
[135] Berkovits N 2006 Super-Poincaré covariant two-loop superstring amplitudes J. High Energy Phys. JHEP01(2006)005

[136] D'Hoker E, Mafra C R, Pioline B and Schlotterer O 2020 Two-loop superstring five-point amplitudes: I. Construction via chiral splitting and pure spinors J. High Energy Phys. JHEP08(2020)135

[137] D'Hoker E, Mafra C R, Pioline B and Schlotterer O 2020 Two-loop superstring five-point amplitudes: II. Low energy expansion and $S$-duality (arXiv:2008.08687 [hep-th])

[138] Tsuchiya A G 2012 On the pole structures of the disconnected part of hyper elliptic $\mathrm{g}$ loop $M$ point super string amplitudes (arXiv:1209.6117 [hep-th])

[139] Tsuchiya A G 2017 On new theta identities of fermion correlation functions on genus $g$ Riemann surfaces (arXiv: 1710.00206 [hep-th])

[140] He S, Schlotterer O and Zhang Y 2018 New BCJ representations for one-loop amplitudes in gauge theories and gravity Nucl. Phys. B $930328-83$ 